University of Louisville

ThinkIR: The University of Louisville's Institutional Repository

Faculty Scholarship

4-2015

\title{
A classical morphological analysis of galaxies in the Spitzer Survey of Stellar Structure in Galaxies (S4G).
}

\author{
Ronald J. Buta \\ University of Alabama - Tuscaloosa \\ Kartik Sheth \\ National Radio Astronomy Observatory \\ E. Athanassoula \\ Aix Marseille Universite \\ Albert Bosma \\ Aix Marseille Universite \\ Johan H. Knapen \\ Universidad de La Laguna \\ See next page for additional authors
}

Follow this and additional works at: https://ir.library.louisville.edu/faculty

Part of the Astrophysics and Astronomy Commons

Original Publication Information

Buta, Ronald J., et al. "A Classical Morphological Analysis of Galaxies in the Spitzer Survey of Stellar Structures in Galaxies (S4G)." 2015. The Astrophysical Journal Supplement Series 217(2): 46 pp.

This Article is brought to you for free and open access by ThinkIR: The University of Louisville's Institutional Repository. It has been accepted for inclusion in Faculty Scholarship by an authorized administrator of ThinkIR: The University of Louisville's Institutional Repository. For more information, please contact thinkir@louisville.edu. 


\section{Authors}

Ronald J. Buta, Kartik Sheth, E. Athanassoula, Albert Bosma, Johan H. Knapen, Eija Laurikainen, Heikki Salo, Debra M. Elmegreen, Luis C. Ho, Dennis Zaritsky, Helene M. Courtois, Joannah Hinz, Juan Carlos Muñoz-Mateos, Taehyun Kim, Michael Regan, Dimitri A. Gadotti, Armando Gil de Paz, Jarkko Laine, Karin Menendez-Delmestre, Sebastien Comeron, Santiago Erroz-Ferrer, Mark Seibert, Trisha Mizusawa, Benne W. Holwerda, and Barry Madore 


\title{
A CLASSICAL MORPHOLOGICAL ANALYSIS OF GALAXIES IN THE SPITZER SURVEY OF STELLAR STRUCTURE IN GALAXIES $\left(S^{4} \mathrm{G}\right)$
}

\author{
Ronald J. Buta ${ }^{1}$, Kartik Sheth $^{2}$, E. Athanassoula ${ }^{3}$, A. Bosma ${ }^{3}$, Johan H. Knapen ${ }^{4,5}$, Eija Laurikainen ${ }^{6,7}$, Heikki Salo ${ }^{6}$, \\ Debra Elmegreen $^{8}$, Luis C. Ho ${ }^{9,10,11}$, Dennis Zaritsky ${ }^{12}$, Helene Courtois ${ }^{13,14}$, Joannah L. Hinz ${ }^{12}$, \\ Juan-Carlos Muñoz-Mateos ${ }^{2,15}$, Taehyun Kim ${ }^{2,15,16}$, Michael W. Regan ${ }^{17}$, Dimitri A. Gadotti ${ }^{15}$, Armando Gil De Paz ${ }^{18}$, \\ Jarkko Laine ${ }^{6}$, Karín Menéndez-Delmestre ${ }^{19}$, Sébastien Comerón ${ }^{6,7}$, Santiago Erroz Ferrer ${ }^{4,5}$, Mark Seibert ${ }^{20}$, \\ Trisha Mizusawa ${ }^{2,21}$, Benne Holwerda ${ }^{22}$, and Barry F. Madore ${ }^{20}$ \\ ${ }^{1}$ Department of Physics and Astronomy, University of Alabama, Box 870324, Tuscaloosa, AL 35487-0324, USA \\ ${ }^{2}$ National Radio Astronomy Observatory/NAASC, 520 Edgemont Road, Charlottesville, VA 22903, USA \\ ${ }^{3}$ Aix Marseille Universite, CNRS, LAM (Laboratoire d'Astrophysique de Marseille) UMR 7326, F-13388, Marseille, France \\ ${ }^{4}$ Departamento de Astrofísica, Universidad de La Laguna, E-38206 La Laguna, Spain \\ 5 Instituto de Astrofísica de Canarias, Vía Láctea s/n E-38205 La Laguna, Spain \\ ${ }^{6}$ Division of Astronomy, Department of Physical Sciences, University of Oulu, Oulu, FI-90014, Finland \\ ${ }^{7}$ Finnish Centre of Astronomy with ESO (FINCA), University of Turku, Vaisalantie 20, FI-21500, Piikio, Finland \\ ${ }^{8}$ Vassar College, Deparment of Physics and Astronomy, Poughkeepsie, NY 12604, USA \\ ${ }^{9}$ Kavli Institute for Astronomy and Astrophysics, Peking University, Beijing 100871, China \\ ${ }^{10}$ Department of Astronomy, Peking University, Beijing 100871, China \\ ${ }^{11}$ The Observatories of the Carnegie Institution for Science, 813 Santa Barbara Street, Pasadena, CA 91101, USA \\ ${ }^{12}$ Steward Observatory, University of Arizona, 933 North Cherry Avenue, Tucson, AZ 85721, USA \\ ${ }^{13}$ Université Lyon 1, CNRS/IN2P3, Institut de Physique Nucléaire, Lyon, France \\ ${ }^{14}$ Institute for Astronomy, University of Hawaii, 2680 Woodlawn Drive, Honolulu, HI 26822, USA \\ ${ }^{15}$ European Southern Observatory, Casilla 19001, Santiago 19, Chile \\ ${ }^{16}$ Astronomy Program, Department of Physics and Astronomy, Seoul National University, Seoul 151-742, Korea \\ ${ }_{17}$ Space Telescope Science Institute, 3700 San Martin Drive, Baltimore, MD 21218, USA \\ ${ }^{18}$ Departmento de Astrofisica, Universidad Complutense de Madrid, E-28040 Madrid, Spain \\ ${ }^{19}$ University of Rio de Janeiro, Observatorio de Valongo, Ladeira Pedro Antonio, 43, CEP 20080-090, Rio de Janeiro, Brazil \\ ${ }^{20}$ The Observatories of the Carnegie Institution for Science, 813 Santa Barbara Street, Pasadena, CA 91101, USA \\ ${ }^{21}$ Department of Physics and Space Sciences, Florida Institute of Technology, 150 W. University Boulevard, Melbourne, FL 32901, USA \\ ${ }^{22}$ University of Leiden, Sterrenwacht Leiden, Niels Bohrweg 2, NL-2333 CA Leiden, The Netherlands \\ Received 2014 July 21; accepted 2014 December 26; published 2015 April 24
}

\begin{abstract}
The Spitzer Survey of Stellar Structure in Galaxies $\left(\mathrm{S}^{4} \mathrm{G}\right)$ is the largest available database of deep, homogeneous middle-infrared (mid-IR) images of galaxies of all types. The survey, which includes 2352 nearby galaxies, reveals galaxy morphology only minimally affected by interstellar extinction. This paper presents an atlas and classifications of $\mathrm{S}^{4} \mathrm{G}$ galaxies in the Comprehensive de Vaucouleurs revised Hubble-Sandage (CVRHS) system. The CVRHS system follows the precepts of classical de Vaucouleurs morphology, modified to include recognition of other features such as inner, outer, and nuclear lenses, nuclear rings, bars, and disks, spheroidal galaxies, $\mathrm{X}$ patterns and box/peanut structures, OLR subclass outer rings and pseudorings, bar ansae and barlenses, parallel sequence latetypes, thick disks, and embedded disks in 3D early-type systems. We show that our CVRHS classifications are internally consistent, and that nearly half of the $\mathrm{S}^{4} \mathrm{G}$ sample consists of extreme late-type systems (mostly bulgeless, pure disk galaxies) in the range Scd-Im. The most common family classification for mid-IR types S0/a to Sc is SA while that for types Scd to Sm is SB. The bars in these two type domains are very different in mid-IR structure and morphology. This paper examines the bar, ring, and type classification fractions in the sample, and also includes several montages of images highlighting the various kinds of "stellar structures" seen in mid-IR galaxy morphology.
\end{abstract}

Key words: atlases - galaxies: elliptical and lenticular - galaxies: irregular - galaxies: spiral - galaxies: structure

Supporting material: figure set, machine-readable tables

\section{INTRODUCTION}

Galaxy morphology and classification are an essential step in understanding how galaxies form and evolve. Morphology is rich in clues to the internal and external physical processes that have molded galactic shapes. It is, however, non-trivial to determine exactly what a given morphology actually implies about the history of a galaxy, because we only see the $z \approx 0$ end-product of all of these processes, whether secular in nature or not. Only by examining the collective morphology of galaxies, both nearby and very distant, in conjunction with physical data (such as luminosities, diameters, inclinations, and bulge properties) and numerical simulations of galaxy evolution, can we hope to piece together the general evolutionary paths of different classes of galaxies.
The Spitzer Space Telescope (Werner et al. 2004) opened a new window on galaxy structure at middle-infrared (mid-IR) wavelengths. With the Infrared Array Camera (IRAC; Fazio et al. 2004), Spitzer provided four major IR bands for direct imaging: $3.6,4.5,5.8$, and $8.0 \mu \mathrm{m} .^{23}$ These bands cover a unique part of the galactic spectrum: the 3.6 and $4.5 \mu \mathrm{m}$ bands largely sample the photospheric light of old stars (Pahre et al. 2004), while the 5.8 and $8.0 \mu \mathrm{m}$ bands reveal the dusty

\footnotetext{
${ }^{23}$ Pahre et al. (2004) considered all four IRAC bands to be mid-IR, while the Infrared Processing and Analysis Center (IPAC) considers the 3.6 and $4.5 \mu \mathrm{m}$ filters as near-IR and the 5.8 and $8.0 \mu \mathrm{m}$ filters as mid-IR (www.ipac.caltech. edu/outreach/Edu/Regions/irregions.html). Here we use mid-IR for all of the IRAC filters to distinguish them from the ground-based near-IR studies of the past that were in the IJHK bands (ranging from 0.8 to $2.2 \mu \mathrm{m}$ ).
} 
interstellar medium (Helou et al. 2004). In all of these bands, star formation and the interstellar medium are evident to various degrees in the form of either emission lines or thermal emission from dust heated by massive stars. Most importantly, these mid-IR bands show galaxies mostly free of the effects of extinction and reddening, revealing previously hidden structures (e.g., rings in edge-on galaxies, or nuclear rings in the dusty central areas of some barred galaxies).

The Spitzer Survey of Stellar Structure in Galaxies $\left(\mathrm{S}^{4} \mathrm{G}\right.$; Sheth et al. 2010) is the largest database of high-quality mid-IR images of nearby galaxies available. Publicly released in 2013, the $S^{4} G$ includes 2352 galaxies imaged in the 3.6 and $4.5 \mu \mathrm{m}$ bands, selected according to redshift, distance, apparent brightness, and galactic latitude. Because these filters are predominantly sensitive to the light of old stars, they trace the distribution of stellar mass. The primary goal of the $\mathrm{S}^{4} \mathrm{G}$ was to "obtain a complete census of the stellar structures in galaxies in the local volume." For this purpose, the images have been used for studies of structures in the faint outskirts of galaxies and of tidal debris (Kim et al. 2012; Laine et al. 2014b), the properties of thick disks seen in edge-on galaxies of types $\mathrm{Sb}$ to $\mathrm{Sdm}$ and profile breaks (Comerón et al. 2011a, 2011b, 2011c, 2012), mid-IR flocculent and grand design spiral structure and starforming regions (Elmegreen et al. 2011, 2014), conversion of 3.6 and $4.5 \mu \mathrm{m}$ light into stellar mass maps (Meidt et al. 2012, 2014; M. Querajeta et al. 2014, in preparation), properties of stellar mass galactic rings (Comerón et al. 2014), bar brightness profiles (Kim et al. 2014), outer disk brightness profiles (Munoz-Mateos et al. 2013; Laine et al. 2014a), quantitative morphology using cosmologically relevant parameters (Holwerda et al. 2014), mid-IR asymmetries and the mid-IR Tully-Fisher relation (Zaritsky et al. 2013, 2014), examination of a possible relation between nuclear activity and bar strength (Cisternas et al. 2013), and for the photometric decompositions of bulges, disks, and bars in the mid-IR (Salo et al. 2015). Knapen et al. (2014) have also gathered high-quality optical images for about $60 \%$ of the survey galaxies.

The $S^{4} \mathrm{G}$ also provides an opportunity to examine the mid-IR structure of a large number of galaxies from the point of view of classical morphological analysis, meaning the classification of galaxies in the well-known Hubble $(1926,1936)$ system and its later offshoots (de Vaucouleurs 1959; Sandage 1961; Sandage \& Bedke 1994). This is worth doing for several reasons: (1) the value of visual classification has increased over the past 20 years owing to the explosion in imaging data available (e. g., the Sloan Digital Sky Survey) as well as to advances in numerical simulations and theoretical understanding of the processes that impact galactic shapes (Athanassoula 2012; Kormendy 2012); (2) Because the midIR provides the clearest view of galactic stellar mass morphology, the symbolism of classical morphological analysis (i.e., $\mathrm{SA}(\mathrm{r}) \mathrm{a}, \mathrm{SB}(\mathrm{s}) \mathrm{bc}$, etc) has more meaning than it did when the $B$ band, the waveband in which galaxy classification has traditionally been performed (as in, e.g., de Vaucouleurs 1959; Sandage 1961; Sandage \& Bedke 1994; Buta et al. 2007), was the only band used for such analysis; (3) the high-quality of $S^{4} G$ images with respect to uniformity, depth of exposure, and resolution in the IR, and the detailed information the images provide on both nuclear and outer structure allows us to improve on the morphological types listed in published catalogs like the Third Reference catalog of
Bright Galaxies (RC3, de Vaucouleurs et al. 1991); (4) examining such a high-quality database at the level of detail needed for classical morphological analysis can draw attention to special cases of interest; (5) classical morphological types in the mid-IR complement the quantitative analyses that are a major part of the $\mathrm{S}^{4} \mathrm{G}$ project (Sheth et al. 2010); (6) specialized visual classifications are still essential to the automated and crowd-sourced classifications that are a common practice in astronomy today, especially for high redshift studies (e.g., Coe et al. 2006; Huertas-Company et al. 2008; Lee et al. 2013); and (7) the large number of images that are homogeneous in sensitivity, coverage, and spatial resolution avoid the problems that would plague heterogeneous datasets.

Buta et al. (2010a, hereafter paper I) presented a preliminary morphological analysis of nearly $200 \mathrm{~S}^{4} \mathrm{G}$ galaxies from the Spitzer archives, and showed that the old $B$-band classification systems could be effectively applied in the mid-IR. This did not mean that there were no problems in the actual application of a $B$-band system in the mid-IR, only that on the whole the classical systems could still be used for the majority of mid-IR galaxy types. Eskridge et al. (2002) came to the same conclusion using near-IR $H$-band $(1.65 \mu \mathrm{m})$ images. $H$-band types are compared with our mid-IR classifications in Section 3.3.

In this paper, we present a similar analysis to paper I of the entire $\mathrm{S}^{4} \mathrm{G}$ sample. We use the notation of the "Comprehensive de Vaucouleurs revised Hubble-Sandage" (CVRHS) system (e.g., Buta 2014) to provide classifications similar to, but more extensive than, those provided in the RC3. Much of the background for the survey is already described in paper I; only a brief summary will be provided here. In addition to an atlas of images of the $2168 \mathrm{~S}^{4} \mathrm{G}$ galaxies not included in the paper I analysis, we highlight specific aspects of mid-IR morphology (as well as interesting individual cases). Because the CVRHS has "evolved" since 2010 to include more features (such as ansae bars and barlenses; Section 4.3) and also uses aspects of the van den Bergh (1976) parallel sequence classification (following developments summarized by Kormendy \& Bender 2012), the present study includes a re-examination of the paper I galaxies.

\section{GALAXY SAMPLE}

The sample selection for the $\mathrm{S}^{4} \mathrm{G}$ is described by Sheth et al. (2010). All galaxies in the Hyperleda database (Paturel et al. 2003) having an $\mathrm{HI}$ radial velocity $\left(V_{\text {radio }}\right)<3000 \mathrm{~km}$ $\mathrm{s}^{-1}$ (corresponding to a distance $D<40 \mathrm{Mpc}$ for $H_{o}=75 \mathrm{~km}$ $\left.\mathrm{s}^{-1} \mathrm{Mpc}^{-1}\right)$, a blue light isophotal diameter $D_{25}>1$ !. $(0)$, a blue photographic magnitude $m_{B}<15.5$ (corrected for internal extinction), and a Galactic latitude of $b>30^{\circ}$ were selected for the survey. The original sample had 2331 galaxies (named in the on-line table accompanying Sheth et al. 2010), but the final sample has 2352 galaxies owing to Spitzer's improved efficiency which allowed 21 galaxies satisfying the original criteria (except for HI detection) to be added. The additional galaxies are primarily HI-poor ellipticals and S0s.

Of the selected galaxies, $\approx 600$ had already been observed by Spitzer for other projects, and thus images were already available. New data were collected for the $\approx 1750$ remaining sample galaxies. The pixel size for each image is 0.75 , achieved using the drizzle technique (Williams et al. 1996) on original images having a pixel size of 1 .'2. The point-spread 
function for the $3.6 \mu \mathrm{m}$ images has a mean full width at half maximum of $1 . " 66$ ( 1.72 for the $4.5 \mu \mathrm{m}$ filter; IRAC Instrument Handbook), which limits the accuracy of the classifications of some of the smaller or more distant galaxies in the sample. The processing of all $\mathrm{S}^{4} \mathrm{G}$ images followed a pipeline with a number of steps (P1-P4) outlined by Sheth et al. (2010).

The use of $21 \mathrm{~cm}$ radial velocities to select $S^{4} G$ sample galaxies introduced a bias against inclusion of gas-poor earlytype galaxies, for which an HI radial velocity would not be available. The bias is being rectified in a supplementary survey (Sheth et al. 2013) of 465 early-type galaxies that satisfy the same selection criteria as the original survey but using an optical radial velocity for the distance limit. With these galaxies, the full $\mathrm{S}^{4} \mathrm{G}$ will include 2817 galaxies. A comparable morphological analysis of these additional galaxies will be provided in a later study.

The images used for our morphological analysis are the Pipeline 1 (P1) images. These are the final, "science ready" mosaics where individual sub-images have been matched with regard to background levels and drizzled to get the final pixel scale. $S^{4} \mathrm{G}$ images are generally much more sensitive to low light levels than are typical ground-based near-IR images, owing to the greatly reduced and much more stable background levels that space observations have compared to ground-based images.

Our approach to galaxy classification from the P1 images is the same as was used in paper I. The final P1 images were background-subtracted and then converted into units of mag $\operatorname{arcsec}^{-2}$ using a common (Vega) zero point of 17.6935. This type of "classification-ready" image has the advantage that all of the galaxies can be displayed in a homogeneous way; with the Vega zero point, the range 11.5-26.5 mag $\operatorname{arcsec}^{-2}$ covered the full range of surface brightnesses for the sample. The same faint limit was used for most of the galaxies, but the bright limit was adjusted for individual objects.

Our final list has 2412 galaxies (NGC 4038 and 4039 are counted as one), 60 more than the extended $\mathrm{S}^{4} \mathrm{G}$ sample. All of the additional galaxies are companions or in the same area as an $S^{4} G$ sample galaxy. As in paper I, we include the full set of classification-ready images as an atlas (Figure 1). Each image was displayed on a 24 bit monitor within an area that is recorded in the caption to the image. Many, but not all, of the additional galaxies are covered in the atlas images.

Smaller or more distant galaxies in the sample are not wellresolved in the IRAC image. Even so, $S^{4} \mathrm{G}$ images are of far higher depth than could ever have been achieved from the ground at near-IR wavelengths, especially for low luminosity, low surface brightness galaxies.

Also as in paper I, only the $3.6 \mu \mathrm{m}$ images were used for the visual classifications presented in this paper. The reason is that these images tend to have a greater depth of exposure (and thus greater sensitivity to stellar mass) than the $4.5 \mu \mathrm{m}$ dataset. With azimuthal averaging of the luminosity distribution, Sheth et al. (2010) showed that these images can detect surface mass densities as low as $\approx 1 M_{\odot} \mathrm{pc}^{-2}$.

Although $3.6 \mu \mathrm{m}$ is an excellent wavelength for seeing the distribution of stellar mass in galaxies, it is not perfect, and indeed no IR band perfectly traces such mass. The main drawbacks of the $3.6 \mu \mathrm{m}$ filter are contamination by "hot dust" and the inclusion of a $3.3 \mu \mathrm{m}$ emission feature due to a polycyclic aromatic hydrocarbon associated with star-forming regions (e.g., Meidt et al. 2012). As shown by Kendall et al.
(2008), hot dust emission at $3.6 \mu \mathrm{m}$ can be removed using an IRAC $8.0 \mu \mathrm{m}$ image if available. However, most $\mathrm{S}^{4} \mathrm{G}$ galaxies do not have an $8.0 \mu \mathrm{m}$ image. Meidt et al. $(2012,2014)$ and $\mathrm{M}$. Querajeta et al. (2014, in preparation) use [3.6]-[4.5] colors and a technique known as "independent component analysis" to locate and remove young contaminants and derive stellar mass maps. Our morphological analysis is based on the original $3.6 \mu \mathrm{m}$ images and not on the corrected stellar mass maps. The main reason for this is that galaxy classification has traditionally been based on the distribution of luminosity and not the distribution of mass.

\section{3. $\mathrm{S}^{4} \mathrm{G}$ MORPHOLOGY}

\subsection{Classification System}

\subsubsection{The VRHS Classification}

The CVRHS system is a modified version of the de Vaucouleurs (1959) revised Hubble-Sandage (VRHS) system that is described in the de Vaucouleurs Atlas of Galaxies (dVA, Buta et al. 2007). More detail on the application of the system, and extensive illustrations of different CVRHS morphological features, is provided in the complementary reviews of Buta (2012; IAC Winter school lectures on morphology and secular evolution) and Buta (2013; phenomenology of galaxy morphology and classification). Table 1 provides a summary of the meaning of the notations of CVRHS morphology used in this paper.

The hallmark of VRHS classification is continuity of structure along three morphological dimensions (in the form of a classification volume); the stage, which refers to the E-S0S-I position along a modified Hubble sequence (the VRHS sequence); the family, referring to the presence or absence of a bar; and the variety, referring to the presence or absence of an inner ring. In addition, there is a fourth dimension known as the outer ring classification, referring to the presence of a large ring in the outer disk. Because stage correlates with several basic physical properties of galaxies (e.g., average surface brightness, color, HI mass-to-blue light ratio), it is considered the fundamental dimension of the system.

The positioning of galaxies in stage depends on specific morphological characteristics: elliptical galaxies are defined by a smoothly declining brightness gradient and little or no evidence for a disk component; S0 galaxies are armless disk galaxies and form a sequence $\left(\mathrm{S}^{-} \rightarrow \mathrm{S}^{\circ} \rightarrow \mathrm{S}^{+}\right)$of increasing structure ranging from subtle inflections in the brightness distribution (type $\mathrm{SO}^{-}$) to prominent rings (type $\mathrm{SO}^{+}$); spirals form a sequence (S0/a-Sa-Sab-Sb-Sbc-Sc-Scd-Sd-Sdm-Sm) of decreasing bulge-to-total luminosity ratio, increasingly open spiral arms, an increasing degree of star formation, and increasing asymmetry; and Magellanic irregular galaxies (Im) are the endpoint characterized by significant asymmetries, often a high degree of scattered star formation, and a significant range in luminosity.

The family classification ranges from SA for nonbarred galaxies to SB for barred galaxies, with an intermediate category of SAB to account for "weakly barred" galaxies, or galaxies intermediate in apparent bar strength between SA and $\mathrm{SB}$. Both relative bar length and bar contrast play a role in family classification. Although bars can be recognized in edgeon spiral galaxies (Section 4.3), reliable family classification is still something that can be done only for low inclination 


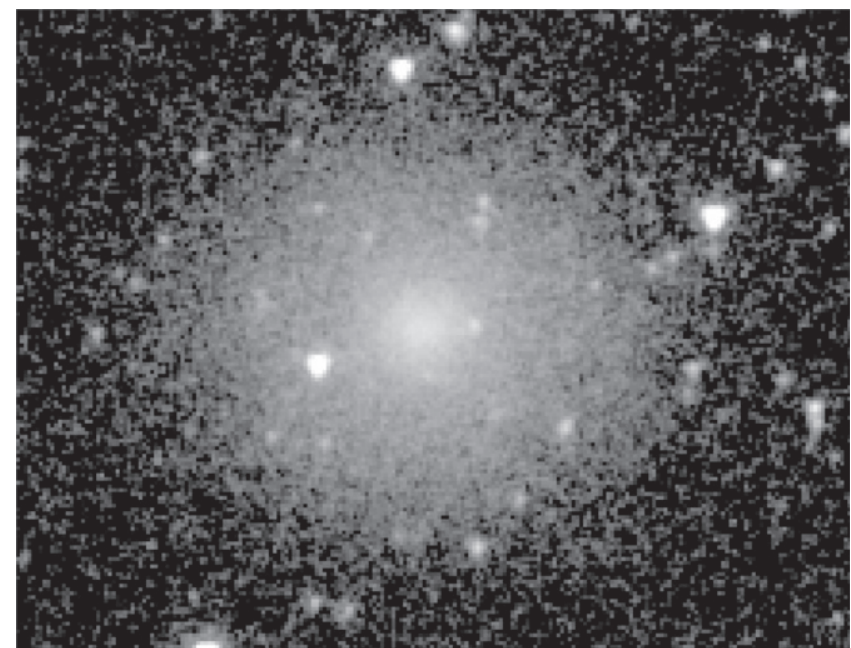

Figure 1. UGC12893; filter: $3.6 \mu \mathrm{m}$; mean (Phase 1, 2) CVRHS type: dSA(1) $0^{\circ} / \mathrm{Sph}$; north up, east left; field: $2.73 \times 2$ ' 10 ; surface brightness range: $18.0-$ $26.5 \mathrm{mag} \mathrm{arcsec}^{-2}$. (Figure 1 is published in its entirety in the electronic edition of the Astrophysical Journal Supplement Series. A portion is shown here for guidance regarding its form and content.)

(The complete figure set (2168 images) is available.)

galaxies. High inclination can considerably foreshorten a bar, or confuse inner structure.

The variety classification ranges from $(r)$ for a closed inner ring to (s) for an open spiral, with an intermediate category (rs) to account for partial inner rings having a spiral character (called "inner pseudorings"). The outer ring classification ranges from $(\mathrm{R})$ for a closed outer ring to $\left(\mathrm{R}^{\prime}\right)$ for an outer pseudoring made of variable pitch angle outer spiral arms. Because outer and inner rings are similar aspects of galaxy morphology, we will henceforth refer to the conventional variety as the "inner variety" and the outer ring classification as the "outer variety" (Section 3.3).

The four parts of a VRHS spiral galaxy classification in order are:

$$
\text { (outer variety)-family-(inner variety)-stage. }
$$

$$
\text { SB }
$$

$\mathrm{ab}$

For example, the VRHS RC3 classification of NGC 1433 is $\left(\mathrm{R}^{\prime}\right) \mathrm{SB}(\mathrm{r}) \mathrm{ab}$. If there is no outer ring or pseudoring, or if the inner variety cannot be determined, these can be dropped from a classification.

The distinction between inner and outer varieties is not always clearcut. Inner and outer rings and pseudorings are easily distinguished in barred galaxies because the bar usually fills an inner ring or pseudoring in one dimension, while outer rings and pseudorings are about twice the bar length in diameter. In the absence of a bar, the distinction may be ambiguous unless more than one ring is present. In some cases, it may not be possible to resolve the ambiguity from visual inspection alone.

The VRHS provides information on inclination through the "spindle" (sp) notation. A spindle is a highly inclined disk galaxy. For example, the VRHS classification for NGC 4565 is $\mathrm{Sb}$ sp. It is too inclined to get a full classification with family and variety, but stage is still distinguishable. The sp after the stage points to its near edge-on orientation.

Peculiarities are recognized using "Pec" as the classification, or "pec" after a regular classification. For example, NGC 4038-
9 is a well-known merger system with distorted components and tidal tails. The object does not fit into any VRHS "cell" and so is classified as "Pec." If instead, "pec" follows a classification, it implies something unusual about the object, often uncharacteristic asymmetry or odd shape.

de Vaucouleurs (1963) modified the VRHS to include underline notation for stage, family, and (inner) variety, where in a combined classification symbol such as Sab, Sbc, Scd, $\mathrm{Sdm}, \mathrm{SAB}$, and (rs), one symbol is underlined to imply that it is "closest to actual type." For example, a classification like SA (s)a $\underline{b}$ would indicate a stage Sab galaxy that is more $\mathrm{Sb}$ than $\mathrm{Sa}, \mathrm{SB}(\mathrm{s}) \underline{\mathrm{c}} \mathrm{d}$ would be a stage Scd galaxy that is more $\mathrm{Sc}$ than $\mathrm{Sd}$, etc. For family, an SAB galaxy shows only a trace of a bar (often merely an oval), while an SAB galaxy is more barred than nonbarred but not as strongly barred as an SB galaxy. Similarly, for variety an (ㅁs) galaxy shows a well-defined inner ring only slightly broken by spiral structure, while an rs galaxy shows only a trace of an inner ring.

Although very useful (and applied in the catalog of Southern Ringed Galaxies CSRG, Buta 1995 and the dVA), for practical reasons underline notation was not used in any of the reference catalogs (RC1, de Vaucouleurs \& de Vaucouleurs 1964; RC2, de Vaucouleurs et al. 1976; and RC3). de Vaucouleurs (1963) also used underline notation sparingly: in his survey of 1500 bright galaxies, including 1263 spirals and S0s, underline notation for stages was used for only $2 \%$ of the galaxies, while underline notation for family and variety was used for only 9-10\% of the galaxies. In VRHS classifications, the bulk of classifications will be in the main categories, while underline categories will generally be underrepresented.

\subsubsection{Comprehensive VRHS Classification}

What the CVRHS adds to the original VRHS system is recognition of details whose significance to galaxy structure and evolution has only recently been appreciated. For example, Kormendy (1979) showed that lenses, disk morphological features characterized by a shallow brightness gradient interior to a sharp edge, are prominent in barred galaxies and could be intimately connected with the evolution of bars. He argued that lenses were often misclassified as rings in $\mathrm{RC} 2$, and noted that there was a lens analog of each type of ring in the VRHS. He suggested using the symbol (1) for inner lenses (analog of inner rings) and (L) for outer lenses (analog of outer rings). ${ }^{24}$ The significance of lenses to galaxy morphology was further established with the near-IR S0 Survey (NIRS0S), a $K_{s}$-band $(2.2 \mu \mathrm{m})$ survey of 206 early-type galaxies, including $160 \mathrm{~S} 0$ S0/a galaxies (Laurikainen et al. 2011, 2013).

An important question is how lenses differ from bulges. In the case of inner lenses, there is no ambiguity between these features because the bar tends to fill the lens in one dimension (Kormendy 1979). However, there is another type of lens, called a "barlens" (Laurikainen et al. 2011) that can be mistaken for a classical bulge (Athanassoula et al. 2014). These are discussed further in Section 4.3.1.

The recognition of lenses brings attention to other features known as ring-lenses, where the apparently sharp edge of a lens is slightly enhanced to appear as a low contrast ring. In CVRHS classification, we use the notation (rl) for an inner

\footnotetext{
${ }^{24}$ Kormendy (2012) prefers the use of (lens) in place of (1) for inner lenses, to avoid possible confusion with (1). Here we continue to use (1) and (L) to be consistent with the dVA and the CSRG.
} 
Table 1

Explanation of CVRHS Symbols ${ }^{\mathrm{a}}$

\begin{tabular}{|c|c|}
\hline Symbol & Description \\
\hline 1 & 2 \\
\hline
\end{tabular}

ETG

ITG

LTG

ETS

ITS

LTS

XLTS

Classical bulge

Pseudobulge

PDG

Stage

E galaxy

En

$\mathrm{E}^{+} \mathrm{n}$

$\mathrm{E} / \mathrm{E}^{+}$

$\mathrm{E}(\mathrm{d}) \mathrm{n}$

$\mathrm{E}(\mathrm{b}) \mathrm{n}$

$\mathrm{E}(\mathrm{b}, \mathrm{nd})$

S0 galaxy

$\mathrm{S}^{-} \rightarrow \mathrm{S}^{\circ} \rightarrow \mathrm{S}^{+}$

$\mathrm{E}^{+} / \mathrm{SO}^{-}$

$\mathrm{SO}^{-}$

$\mathrm{S}^{-/ \mathrm{o}}$

$\mathrm{S}^{\circ}$

$\mathrm{S} 0^{\circ}[\mathrm{d}]$

$\mathrm{S}^{\mathrm{o} /+}$

$\mathrm{S}^{+}$

$\mathrm{SO}^{+}[\mathrm{c}]$

Spiral galaxy

$\mathrm{S} 0 / \mathrm{a}-\mathrm{Sa}-\mathrm{Sb}-\mathrm{Sc}-\mathrm{Sd}-\mathrm{Sm}$

Irregular galaxy

$\mathrm{S} \underline{0} / \mathrm{a}$

$\mathrm{S} 0 / \mathrm{a}$

$\mathrm{S} 0 / \underline{\mathrm{a}}$

$\mathrm{Sa}$

Sa $b$

$\mathrm{Sab}$

$\mathrm{Sa} \underline{b}$

$\mathrm{Sb}$

S $\underline{b} c$

$\mathrm{Sbc}$
An early-type galaxy, collectively referring to a galaxy in the range of types E-Sa

An intermediate-type galaxy, taken to be in the range Sab-Sbc

A late-type galaxy, collectively referring to a galaxy in the range of types Sc-Im

An early-type spiral, taken to be in the range S0/a-Sa

An intermediate-type spiral, taken to be in the range Sab-Sbc

A late-type spiral, taken to be in the range Sc-Scd

An extreme late-type spiral, taken to be in the range $\mathrm{Sd}-\mathrm{Sm}$

A galaxy bulge that likely formed from early mergers of smaller galaxies (Kormendy \& Kennicutt 2004; Athanassoula 2005)

A galaxy bulge made of disk material that has secularly collected into the central regions of a barred galaxy (Kormendy 2012)

A pure disk galaxy, a galaxy lacking a classical bulge and often also lacking a pseudobulge

\section{Stage}

The characteristic of galaxy morphology that recognizes development of structure, the widespread distribution of star formation, and the relative importance of a bulge component along a sequence that correlates well with basic characteristics such as integrated color, average surface brightness, and HI mass-to-blue luminosity ratio

\section{Elliptical Galaxies}

A galaxy having a smoothly declining brightness distribution with little or no evidence of a disk component and no inflections (such as lenses) in the luminosity distribution (examples: NGC 1052, 3193, 4472)

An elliptical galaxy of visual flattening $n=10(1 \mathrm{~b} / a)$, where $b / a$ is the visual isophotal axis ratio (Hubble 1926)

A "late" elliptical of visual flattening $n$, a transition stage to the S0 class (de Vaucouleurs 1959); show slight traces of differentiated structure, usually subtle evidence of lenses (example: NGC 5846) or a faint outer envelope; also has been used as a "home" for Morgan cD galaxies in RC3

An E galaxy that in our Phase 1, 2 analysis averages between $\mathrm{E}$ and $\mathrm{E}^{+}$(example: NGC 3226)

A disky elliptical galaxy of visual flattening $n$ (Kormendy \& Bender 1996); a subclassification of ellipticals having pointy outer isophotes that is visually detectable only for the most obvious or most favorably oriented cases (example: NGC 3377)

A boxy elliptical galaxy of visual flattening $n$ (Kormendy \& Bender 1996); a subclassification of ellipticals having boxy outer isophotes that is visually detectable only for the most obvious cases

A boxy E galaxy with an inner disk (example: NGC 4370)

\section{SO Galaxies}

A disk-shaped galaxy lacking strong or obvious spiral structure; at minimum, a two-component system with a bulge and a disk a stage sequence of S0 galaxies based on increasing development of structure, such as bars, lenses, and rings

An ETG that in our Phase 1, 2 analysis averages between $\mathrm{E}^{+}$and $\mathrm{S}^{-}$(example: $\mathrm{NGC} \mathrm{4649)}$

An early S0 showing clear evidence for a disk (envelope) but little structure; all features are subtle (examples: NGC 4442, 5507)

An S0 galaxy that in our Phase 1, 2 analysis averages between $\mathrm{S}^{-}$and $\mathrm{S}^{\circ}$

An intermediate stage S0 showing clear lenses or traces of rings/pseudorings (examples: NGC 1411, 1533)

One example of a notation used for an apparently early-type galaxy in the catalog having little or no apparent bulge; other examples include $[\mathrm{c}],[\mathrm{cd}],[\mathrm{m}]$. These are related to the van den Bergh (1976) parallel-sequence Hubble classification system, although that system is not fully built into the catalog (example: NGC 693)

An S0 galaxy that in our Phase 1, 2 analysis averages between $\mathrm{S}^{\circ}$ and $\mathrm{S}^{+}$

A late S0 stage showing strong rings and bars, and in some cases trace spiral structure (examples: NGC 1291, 1326, 4138)

A late S0 with an Sc-like central concentration (examples: NGC 4344, 4451)

Spiral and Irregular Galaxies

A galaxy where a spiral pattern is a major part of the morphology

A stage sequence for spirals (with intermediate types Sab, Sbc, Scd, and Sdm) based on Hubble's three criteria: relative prominence of the bulge, the degree of openness of the arms, and the degree of resolution of the arms into star clusters or very luminous stars

A complex system characterized by an irregular distribution of star formation; this irregular distribution can, however, be embedded in a more regular background

An S0/a galaxy that is closer to $\mathrm{S}^{+}$than to Sa (examples: NGC 522, 2681, 3626)

A transition stage showing clear but subtle tightly wrapped spiral arms; structure is generally smooth but trace star formation is seen in nearby examples (examples: NGC 1350, 1452, 4394, 4454, 4984, 5701, 6340, 7098)

An S0/a galaxy that is closer to Sa than to $\mathrm{SO}^{+}$(examples: NGC 3185,3900 )

An early-type spiral, usually defined by relatively smooth, tightly wrapped spiral arms and a significant bulge; standard interpretation may be violated in a cluster environment or in presence of a bar (examples: NGC 1433, 1512, 3031, 3788, 4260, 4450, 4548, 4800, 7513) An Sab galaxy that is closer to Sa than to Sb (example: NGC 2985)

An intermediate-type spiral galaxy similar to Sa but with a more knotty structure (examples: NGC 210, 1097, 3992, 4995; IC 1993)

An Sab galaxy that is closer to Sb than to Sa (examples: NGC 3177, 4902)

An intermediate-type spiral having relatively more open, knotty arms and a smaller bulge than Sa or Sab galaxies (examples: NGC 908, 3433, 3689, 3705, 4237, 7479)

An Sbc galaxy that is closer to Sb than to Sc (examples: NGC 3344, 3512; IC 769)

Typically, an intermediate-type galaxy having well-developed, open, knotty spiral arms like an Sc galaxy but with a more significant bulge (examples: NGC 1365, 3184, 3198, 3338, 3726) 
Table 1

(Continued)

\begin{tabular}{ccc}
\hline \hline & $\begin{array}{c}\text { Symbol } \\
1\end{array}$ & Description \\
2 & \\
\hline Sb $\underline{c}$ & & An Sbc galaxy that is closer to Sc than to Sb (examples: NGC 2715, 4303)
\end{tabular}

$\mathrm{Sc}$

Sc d

Scd

Scd

$\mathrm{Sd}$

S $\underline{d m}$

$\mathrm{Sdm}$

Sdm

$\mathrm{Sm}$

$\mathrm{S} / \mathrm{Im}$

Im

$\operatorname{Im}(\mathrm{cc})$

I0

$\mathrm{dE}$

dS0

dE, $N$

dS0, $N$

dIm

Sph

Sph, $N$

BCD

Family

SA

SÁB

SAB

SAB

$\mathrm{SB}$

$\mathrm{SAB}_{a}, \mathrm{SB}_{a}$

$\mathrm{SAB}_{\mathrm{x}}, \mathrm{SB}_{\mathrm{x}}$

$\mathrm{SAB}_{x a}, \mathrm{SB}_{x a}$

Inner variety

(r)

(rs)

(rs)

$(\mathrm{rs})$
A late-type spiral having well-developed, open, and knotty spiral arms with a small but significant bulge (examples: NGC 1042, 1084, 3486, 3810, 3893, 4411 B, 5457, 5970, 7448; IC 1953)

An Scd galaxy that is closer to Sc than to Sd (examples: NGC 1073, 5033, 5468)

Similar to an Sc but with little or no bulge; typically a small central object (nuclear star cluster or pseudobulge) may be seen; arms can be more open than for an Sc; these are generally pure disk galaxies (PDGs; examples: NGC 1255, 1559, 3346, 5334, 5595, 7741)

An Scd galaxy that is closer to Sd than to Sc (examples: NGC 255, 1253, 3359, 4411 A, 5597, 5668)

An extreme late-type spiral, similar to Scd but with less apparent central concentration than an Scd; among the most common PDGs; asymmetry is often present but less extreme than in Sdm or Sm,Im types (examples: NGC 3003, 4294, 4731, 5068, 5669)

An Sdm galaxy that is closer to Sd than to Sm (examples: NGC 247, 3556, 7151, 7497)

An extreme late-type spiral showing considerable asymmetry, usually with one arm longer and better defined than the other; considerable star formation also characterizes these PDGs (examples: NGC 300, 3906, 4395, 4630, 7154)

An Sdm galaxy that is closer to Sm than to Sd (example: NGC 2552)

A magellanic spiral, usually characterized by a single spiral arm emerging from a bar or central region and little or no bulge or central concentration (de Vaucouleurs \& Freeman 1972; examples: NGC 55, 5474, 7091)

A magellanic galaxy that is closer to Im than to Sm (example: NGC 4353)

A magellanic irregular galaxy, characterized by an irregular shape sometimes within a smooth background of starlight; often show considerable star formation and a wide range of luminosities (examples: NGC 4214, 4242, 4449, 4605)

A clump cluster, usually referring to an irregular galaxy with a large number of scattered star forming regions. The term was originally applied to high redshift clumpy galaxies (e.g., Elmegreen et al. 2009; examples: IC 1826, 2040; UGC 1945)

A type of galaxy seen mainly in blue light images where a highly irregular dust distribution is seen within an S0 or E-like background; at $3.6 \mu \mathrm{m}$, I0 galaxies are relatively normal-looking ETGs (examples: NGC 2968, 3077, 5195, 5253, 5363)

Dwarf and Spheroidal Galaxies

A "dwarf elliptical" galaxy, defined to have a "smooth intensity distribution over the face and ... low surface brightness" (Sandage \& Binggeli 1984; Non-Virgo example in $\mathrm{S}^{4} \mathrm{G}$ catalog: NGC 59)

A "dwarf S0" galaxy, a class of dwarfs which resemble dE galaxies except for "a change of slope in the radial gradient of the light distribution" showing "either direct evidence of a disk, or ... evidence of two components" (Sandage \& Binggeli 1984)

A nucleated dwarf E galaxy (Sandage \& Binggeli 1984)

A nucleated dwarf S0 galaxy (Sandage \& Binggeli 1984)

A dwarf irregular galaxy, typically an Im galaxy having an absolute $B$-band magnitude $M_{B}^{o} \gtrsim-17$; low surface brightness and often resolved in $\mathrm{S}^{4} \mathrm{G}$ images

A "spheroidal" galaxy, a type of galaxy having the appearance of an E or S0 galaxy, but the photometric characteristics of much later-type, lower luminosity galaxies (like Sm, Im types); the dE and dS0 galaxies in the Virgo Cluster are all of this basic type (Kormendy \& Bender 2012)

A nucleated spheroidal galaxy (Binggeli et al. 1985; Kormendy et al. 2009)

A star-forming, blue compact dwarf galaxy; at $3.6 \mu \mathrm{m}$, can appear as a dE or dS0 galaxy (example: NGC 1705)

Family

The characteristic of galaxy morphology that recognizes the apparent strength of a bar or other type of nonaxisymmetric structure, such as an oval

A nonbarred spiral or S0 galaxy (examples: NGC 488, 628, 1411, 4698; IC 1993, 5267)

A galaxy showing a trace of a bar, usually in the form of a broad oval or a very low contrast regular bar (examples: NGC 4203, 4899)

A barred galaxy of intermediate apparent bar strength (examples: NGC 4535, 5236, 7743)

A bar that is clear and well-defined but weaker-looking than a typical SB galaxy bar (examples: NGC 4639, 4818, 5566, 5701)

A barred galaxy with a conspicuous bar, usually strong and obvious (examples: NGC 1300, 1365, 1452, 7513)

A barred galaxy where the bar is defined by brightness enhancements ("ansae"; Sandage 1961; Danby 1965) at its ends; these enhancements may be round spots, short linear features, arcs, or star-forming clumps (Buta 2013; examples: NGC 2787, 5375, 7098 see Figures 12 and 13 for others)

A galaxy showing a prominent $\mathrm{X}$ or box/peanut structure in its inner regions; often seen in edge-on galaxies, the box/peanut/X shape is believed to be a manifestation of vertical resonant orbits in a bar potential, thus it indicates the presence of a bar (Athanassoula 2005). An X may also be seen in the clear bars of non-edge galaxies (Buta et al. 2007; Erwin \& Debattista 2013; examples: NGC 2654 Figure 14; nearly edge-on; NGC 5377 intermediate inclination)

A galaxy showing both an $\mathrm{X}$ and a pair of ansae; the $\mathrm{X}$ is usually three-dimensional while the ansae are flat. (example: NGC 4216 Figures 12 and 13)

\section{Standard Inner Varieties}

The characteristic of galaxy morphology that recognizes the presence or absence of an inner ring

An inner ring, a closed circular or oval feature enveloping either the ends of a bar if present or the central bulge (examples: NGC 1433, $3351,3486,4245,5566)$

A well-defined inner ring, but made of tightly wrapped spiral structure (examples: NGC 613, 1398, 3368, 3705)

An inner "pseudoring," a partial inner ring made of spiral arms (examples: NGC 779, 1232, 3346, 4548)

A weak inner pseudoring, usually fairly open and characterized by a pitch angle only a little different from that of the outer spiral arms (examples: NGC 1365, 3513, 4501, 4548, 5383) 
Table 1

(Continued)

\begin{tabular}{l}
\hline \hline \\
$\begin{array}{c}\text { Symbol } \\
1\end{array}$ \\
\hline$(\mathrm{s})$ \\
$(\mathrm{rr})$ \\
$\left(x_{1} \mathrm{r}\right)$ \\
$(\mathrm{r}, \mathrm{s})$ \\
$(\mathrm{rs}, \mathrm{rs})$ \\
$(\mathrm{s}, \mathrm{rs})$ \\
$(\mathrm{l})$ \\
$(\mathrm{rl})$ \\
$(\mathrm{rs}, \mathrm{rl})$ \\
$(\mathrm{r}$ 'l) \\
$(\mathrm{ls})$ \\
$(\mathrm{p})$ \\
$(\mathrm{bl})$
\end{tabular}

A galaxy having two inner rings (example: NGC 4698)

A ring-like feature that outlines a bar, possibly related to the $x_{1}$ family of bar orbits discussed by Regan \& Teuben (2004; example: NGC 6012)

A variety where the inside of an inner ring includes a spiral pattern unrelated to the main outer spiral arms; prototype in this catalog is NGC 5364

A galaxy having two inner pseudorings, usually of very different sizes (examples: NGC 289, 4689)

A galaxy having a strong s-shaped spiral in the presence of an inner pseudoring (example: NGC 986)

An inner lens, a type of feature, often seen in S0 galaxies, having a shallow brightness gradient interior to a sharp edge (Kormendy 1979; examples: NGC 1291, 1411, 4269, 5602)

An inner ring-lens, recognized as a low contrast inner ring; types (ㅁl) and (rl) recognize different degrees of contrast enhancement (examples: NGC 2859, 4250)

A galaxy having an inner pseudoring and an inner ring-lens, the latter the smaller (example: NGC 5055)

An inner pseudoring-lens, where the inner ring shows azimuthal contrast differences like spiral arms (examples: NGC 210, 1415, 3147)

An inner lens with a subtle embedded spiral pattern (example: NGC 3675)

A "plume," usually seen as a secondary spiral arc positioned just off the leading sides of a bright inner ring (Buta 1984); much rarer than inner rings or lenses (example: NGC 1433)

A "barlens," a feature recognized by Laurikainen et al. 2011, 2013, 2014) and Athanassoula et al. (2014) as the inner part of an early-type bar [examples: NGC 1433, 2787, 3351 (see Figure 11)]

\section{Nuclear Varieties}

The nuclear variety classification refers to rings, lenses, bars, and other features that are often found in the centers of barred galaxies, but which may also be found in nonbarred galaxies

A nuclear (or circumnuclear) ring, a small star-forming feature found in the centers of barred galaxies but also sometimes seen in nonbarred galaxies (example: NGC 1097; see Figure 20 for others)

A nuclear pseudoring, where the ring appears formed by a wrapped spiral pattern; in optical images, this character may sometimes be an artifact of inner dust lanes in bars (examples: NGC 1068, 1090)

A nuclear spiral, a type of feature that may also be an artifact of dust in optical bands (example: NGC 1022)

A nuclear lens; an excellent example in the $\mathrm{S}^{4} \mathrm{G}$ catalog is NGC 4250

A nuclear ring-lens (examples: NGC 210, 1300, 1433, 5566)

A nuclear (or secondary) bar, a feature found in the centers of barred galaxies but which may also be found in SA galaxies (examples: NGC 1291, 1433, 4725)

A nuclear ansae-type bar

A nuclear disk, usually seen in well-resolved edge-on disk galaxies (examples: NGC 24, 678, 1532, 3079, 3628, 4111, 5907)

a "nuclear pattern," a nuclear structure of uncertain nature

a triaxial bulge, an elongated central component whose major axis is misaligned with the galaxy major axis

a pseudobulge, an elongated bulge component whose major axis is approximately aligned with the disk major axis (example: NGC 4536) Combined Inner and Nuclear Varieties ${ }^{b}$

A galaxy having both an inner ring and a much smaller nuclear ring (examples: NGC 1326, 1512, 3351, 4274, 5850)

A galaxy having both an inner lens and a nuclear lens (example: NGC 1411)

A galaxy having an inner pseudoring, a nuclear ring, and a nuclear bar (example: NGC 4321)

A galaxy having an inner ring-lens (more lens than ring), a barlens, and a nuclear ring (example: NGC 4314)

\section{Standard Outer Varieties}

The characteristic of galaxy morphology which recognizes a large ring or ring-like pattern in the outer regions of a galaxy

An outer ring, a closed ring-shaped feature usually about twice the size of a bar in barred galaxies (examples: NGC 1291, 1350, 2859)

An outer "pseudoring," a near-outer ring made of spiral arms (examples: NGC 986, 1300, 1365, 5757, 7479)

\section{Special Outer Varieties ${ }^{\mathrm{b}}$}

A galaxy having two outer rings, usually of very different sizes (examples: NGC 3898, 4457)

A galaxy having two outer pseudorings, usually of very different sizes (examples: NGC 1425; UGC 8155; PGC 53093)

A galaxy with an outer pseudoring and an outer ring, the latter being the smaller (example: NGC 4984)

An outer lens, a type of feature, often seen in early-type galaxies, that is an outer analog of an inner lens (Kormendy 1979; examples: NGC 2787, 4262)

An outer ring-lens, recognized as a low contrast outer ring; types $(\underline{R} L)$ and $(R \underline{L})$ recognize different degrees of contrast enhancement (examples: NGC 5602, 5750)

A galaxy with an outer ring-lens and an outer ring, the latter being the smaller (example: NGC 3626)

A galaxy with an outer ring-lens and an outer pseudoring, the latter being the smaller (examples: NGC 1367, 4351, 4826)

An outer pseudoring-lens, where the outer ring shows azimuthal contrast differences like spiral arms (examples: NGC 1357, 2780)

"Outer Lindblad Resonance" Morphologies

A closed outer ring showing a shape resembling a broad figure 8; a subtly dimpled oval ring, recognized as an OLR subclass (Buta \& Crocker 1991; Buta 1995; examples: NGC 1326, 4250, 5728; IC 1438, 4214) 
Table 1

(Continued)

\begin{tabular}{|c|c|}
\hline $\begin{array}{l}\text { Symbol } \\
1\end{array}$ & $\begin{array}{l}\text { Description } \\
\quad 2\end{array}$ \\
\hline$\left(\mathrm{R}_{1} \mathrm{~L}\right)$ & An $R_{1}$ outer ring-lens (examples: NGC 2893, 5448) \\
\hline$\left(\mathrm{R}_{1}^{\prime}\right)$ & $\begin{array}{l}\text { An outer pseudoring made from arms that wind about } 180^{\circ} \text { with respect to the bar ends; an OLR subclass (Buta \& Crocker 1991; } \\
\text { Buta 1995; examples: NGC } 1566,3504,4192,4314,5377,7051 \text { ) }\end{array}$ \\
\hline$\left(\mathrm{R}_{2}^{\prime}\right)$ & $\begin{array}{l}\text { An outer pseudoring made from arms that wind about } 270^{\circ} \text { with respect to the bar ends; an OLR subclass (Buta \& Crocker 1991; } \\
\text { Buta 1995; examples: NGC 2633, 7741, ESO 26-1) }\end{array}$ \\
\hline$\left(\mathrm{R}_{1}{ }^{\prime} \mathrm{L}\right)$ & An $\left(\mathrm{R}_{1}{ }^{\prime}\right)$ outer pseudoring-lens (example: NGC 4045$)$ \\
\hline$\left(\mathrm{R}_{2}{ }^{\prime} \mathrm{L}\right)$ & An $\left(\mathrm{R}_{2}{ }^{\prime}\right)$ outer pseudoring-lens (example: NGC 210$)$ \\
\hline$\left(\mathrm{R}_{1} \mathrm{R}_{2}^{\prime}\right)$ & $\begin{array}{l}\text { A combined outer ring-pseudoring pattern where the arms forming the } \mathrm{R}_{2}^{\prime} \text { ring break from an } \mathrm{R}_{1} \text { ring; an OLR subclass (Buta \& } \\
\text { Crocker 1991; Buta 1995; example: NGC 5101) }\end{array}$ \\
\hline$\left(\mathrm{R}_{12}{ }^{\prime}\right)$ & $\begin{array}{c}\text { An unusual version of }\left(\mathrm{R}_{1} \mathrm{R}_{2}{ }^{\prime}\right) \text { where only half of each feature is seen. } \\
\text { Cataclysmic Rings }{ }^{b}\end{array}$ \\
\hline Cataclysmic ring & A ring-like feature formed in a catastrophic galaxy interaction event, such as a head-on collision or major disruption \\
\hline Extraplanar disk & A general term for any acquired disk-shaped feature inclined at an angle to a more massive disk system \\
\hline RG & $\begin{array}{l}\text { A ring galaxy, a term referring to the unusual rings thought to be produced in special galactic collisions (Theys \& Spiegel 1976; Madore } \\
\text { et al. 2009) }\end{array}$ \\
\hline PRG & $\begin{array}{l}\text { A polar ring galaxy or related type of object (Whitmore et al. 1990). In the strictest sense, an interacting system where a companion is } \\
\text { disrupted along a polar or near polar orbit around another disk-shaped system (example: NGC 5122) }\end{array}$ \\
\hline IRG & An inclined ring galaxy, where a companion has been disrupted into a high angle but not necessarily polar orbit (example: NGC 660) \\
\hline$(\mathrm{R}) \mathrm{E}$ & $\begin{array}{l}\text { A likely accretion ring formed from a companion disrupted into a large orbit around an elliptical galaxy (Schweizer et al. 1987) } \\
\text { Edge-on Galaxies }{ }^{\text {c }}\end{array}$ \\
\hline sp & A “spindle," or highly inclined (generally $i>65^{\circ}$ ) disk-shaped galaxy \\
\hline Scd sp & A highly inclined Scd galaxy, too close to edge-on for the family or variety to be reliably distinguished (example: NGC 100) \\
\hline $\mathrm{SB}(\underline{\mathrm{r}} \mathrm{s}) \mathrm{bc} \mathrm{sp}$ & A highly inclined galaxy, but sufficiently far from edge-on that the family and variety are distinguishable (example: NGC 253) \\
\hline spw & $\begin{array}{l}\text { A highly inclined disk-shaped galaxy where the disk shows evidence of warping in the nearly edge-on view (examples: NGC 522, 5084, } \\
\text { 5403; UGC } 10043 \text { Figure 18) }\end{array}$ \\
\hline $\mathrm{S} 0^{-} \mathrm{sp} / \mathrm{E}(\mathrm{d}) 7$ & $\begin{array}{l}\text { An edge-on early S0 galaxy, having a disky thick disk of flattening E7 (example: NGC 1032); E(d) is Kormendy \& Bender (1996) } \\
\text { notation and is used here only in a descriptive manner }\end{array}$ \\
\hline $\mathrm{S} 0^{-} \mathrm{sp} / \mathrm{E}(\mathrm{b}) 6$ & $\begin{array}{l}\text { An edge-on early S0 galaxy, having a boxy thick disk of flattening E6 (example: NGC 1332); E(b) is Kormendy \& Bender (1996) } \\
\text { notation and is used here only in a descriptive manner }\end{array}$ \\
\hline $\mathrm{S}^{-} \mathrm{sp} / \mathrm{E} 5-6$ & An edge-on early S0 galaxy, having an E-like thick disk (neither boxy nor disky) of flattening E5-6 (example: NGC 3115) \\
\hline $\mathrm{Sb} \mathrm{sp} / \mathrm{E} 5$ & An edge-on Sb galaxy embedded in an E5 background (example: NGC 5078) \\
\hline $\mathrm{SA}(\mathrm{rl}, \mathrm{nrl}, \mathrm{nb}) 0^{+} / \mathrm{E}(\mathrm{d}) 0$ & $\begin{array}{c}\text { An inclined disk with multiple features embedded within an E0 background (example: NGC 1553) } \\
\text { Other Special Features or Categories }\end{array}$ \\
\hline Pec & $\begin{array}{l}\text { A peculiar galaxy, a significantly disturbed interacting system that cannot be classified in any other way; typically an ongoing or advanced } \\
\text { merger or other disturbed type of system (examples: NGC 520,4038-9) }\end{array}$ \\
\hline pec & $\begin{array}{l}\text { In a classification, this refers to a classifiable galaxy having some peculiarity (such as some types of asymmetry; examples: NGC } \\
1087,1097)\end{array}$ \\
\hline Dark-spacer & $\begin{array}{l}\text { A galaxy which shows a distinctive area or areas of lower surface brighter within the bright part of a disk (Buta 2014). In some early-type } \\
\text { galaxies, the appearance of an inner ring may be defined mainly by a subtle darker area around a bar (Laurikainen et al. 2013; example: } \\
\text { NGC 2787) }\end{array}$ \\
\hline Counter-winding spiral & A spiral galaxy having two sets of spiral structure which wind outward in opposite senses \\
\hline
\end{tabular}

\footnotetext{
${ }^{\text {a }}$ Examples are all based on Table 6 mean classifications.

${ }^{\mathrm{b}}$ Previously called catastrophic rings by Buta (2013)

${ }^{\mathrm{c}}$ For other combinations, see Table 6.
}

ring-lens and (RL) for an outer ring-lens, with pseudoring-lens equivalents of $\left(r^{\prime} 1\right)$ and $\left(R^{\prime} L\right)$, respectively. These are also used with underline notation to emphasize the ring or lens aspect.

The CVRHS includes recognition of important nuclear features, such as nuclear rings, pseudorings, lenses, ring-lenses, bars, and disks (Buta \& Combes 1996). A nuclear ring (nr) is a small ring, often defined by star-forming regions, typically found in the centers of barred galaxies and on average $\approx 1 \mathrm{kpc}$ in diameter (Comerón et al. 2010). A nuclear lens (nl) is the lens analog of a nuclear ring. A nuclear bar (nb) is a small bar often found within a nuclear ring or lens, or which may be present independent of these features. A nuclear disk (nd) is typically a distinct, highly flattened feature seen most easily in edge-on S0 galaxies. Nuclear ring-lenses (nrl), nuclear pseudorings $\left(\mathrm{nr}^{\prime}\right)$, and nuclear spirals (ns) are also known. Similar to inner and outer rings, in CVRHS classification the presence or absence of a nuclear ring or related feature will be referred to as the "nuclear variety."

The fact that there are three ring types $(\mathrm{R}, \mathrm{r}, \mathrm{nr})$ and three types of lenses $(\mathrm{L}, \mathrm{l}, \mathrm{nl})$, with ring-lenses ( $\mathrm{RL}, \mathrm{rl}, \mathrm{nrl}$ ) as intermediate categories, all with a similar relationship to bar extent, could imply a close connection between rings and lenses in a dynamical or even evolutionary sense. Kormendy (1979) originally argued that inner lenses could be the result of secular dissolution of a primary bar. Comerón (2013) has most recently examined the ring-lens issue and concluded that once a star-forming ring exhausts or is stripped of its gas, it may dissolve into a ring-lens in as little as $200 \mathrm{Myr}$. 
Other characteristics considered part of CVRHS morphology include X-patterns, boxy/disky structures, outer Lindblad resonance (OLR) ring morphologies, warps, spheroidal galaxies, and other features described in more detail in the next Sections. CVRHS morphology also considers alternative points of view, such as the parallel sequence classification of van den Bergh (1976) where S0 galaxies are stripped spirals on a sequence parallel to regular spirals. Three recent studies have provided considerable support for this idea: Laurikainen et al. (2011, 2013), Cappellari et al. (2011), and Kormendy \& Bender (2012). In a cluster environment, parallel sequence classification is a more accurate view of galaxy morphology than the VRHS sequence, and provides a natural home for what Kormendy (2012) refers to as "spheroidal galaxies." Nevertheless, this does not negate the value of CVRHS morphology because CVRHS classification is, for the most part, purely morphological and not based on a "whiff of theory" (Sandage 2005). (An exception is the OLR outer ring/pseudoring morphological subclasses (Buta \& Crocker 1991), which were theoretically predicted by Schwarz (1981).)

Paper I described the application of the CVRHS to $200 \mathrm{~S}^{4} \mathrm{G}$ galaxies, where it was shown that, in spite of the significant differences between modern mid-IR digital images and the optical photographic blue-light plates that were the historical basis for the original VRHS system, many galaxies show the same essential morphological features in the mid-IR as in the $B$ band, allowing the effective application of the VRHS system to $S^{4} \mathrm{G}$ images. There is no need to invent a new classification system to accomodate mid-IR galaxy morphology; instead, it should be sufficient to build on what we already have from studies of blue-light images. This does not mean that the "essential features" are defined in the same way in the two wavebands. For example, the degree of resolution into starforming regions was one of Hubble's original criteria for classifying spiral galaxies into $\mathrm{Sa}-\mathrm{Sb}-\mathrm{Sc}$ bins. In the $B$ band, this resolution is determined by the distribution of aggregates of young blue supergiants. In the mid-IR, however, these stars are not prominent. Instead, we see the thermal emission from the local dust heated by these stars. Paper I also noted that when CVRHS mid-IR stages were compared to RC3 stages, galaxies of types $\mathrm{Sc}$ and later or $\mathrm{S}^{+}$and earlier were often classified the same as in RC3, while intermediate stages such as $\mathrm{S} 0 / \mathrm{a}$ to Sbc were classified about one stage earlier.

Even if it is possible to use an existing classification system effectively in the 3.6 and $4.5 \mu \mathrm{m}$ IRAC bands, the classification of galaxies based on $\mathrm{S}^{4} \mathrm{G}$ mid-IR images can be difficult because of competing factors. For example, the contrast of the spiral structure in mid-IR light can be very low compared to the brightness of the background disk light. This can make it difficult to fit some galaxies into the CVRHS system, especially if a galaxy is small or distant enough to be poorly resolved. Another issue is the greater sensitivity of the IRAC bands to old stellar population bulges (also known as classical bulges Kormendy \& Kennicutt 2004; Athanassoula 2005) while at the same time being less sensitive to spiral structure.

The galaxies that show the most drastic differences between mid-IR and $B$-band morphology are usually those having considerable internal dust extinction, such as edge-on spirals, starburst galaxies, and major or minor merger systems. Extinction in the mid-IR bands is less than $5 \%$ of that in the $B$ band, and generally allows fairly good penetration into thick planar dust layers. $\mathrm{S}^{4} \mathrm{G}$ images do allow us to improve our
Table 2

Phase 1 and 2 CVRHS Classifications for $2412 \mathrm{~S}^{4} \mathrm{G}$ Galaxies ${ }^{\mathrm{a}}$

\begin{tabular}{|c|c|c|c|}
\hline Galaxy & Phase 1 Type & Phase 2 Type & Figure No. \\
\hline 1 & 2 & 3 & 4 \\
\hline \multicolumn{4}{|l|}{$\rightarrow$ R.A.: $0^{h} \leftarrow$} \\
\hline UGC 12893 & $\mathrm{dSA}(1) 0^{\circ} / \mathrm{Sph}$ & $\mathrm{dSA}(1) 0^{\circ} / \mathrm{Sph}$ & II.1.0001 \\
\hline PGC 143 & $\mathrm{dIm}$ & $\mathrm{dI}$ & II.1.0002 \\
\hline ESO 12-14 & $\mathrm{IB}(\mathrm{s}) \mathrm{m}$ & $\mathrm{SB}(\mathrm{s}) \mathrm{m}$ & II.1.0003 \\
\hline UGC 17 & IABm & IABm & II.1.0004 \\
\hline NGC 7814 & SA0/a spw & SA0/a spw & II.1.0005 \\
\hline NGC 7817 & $\mathrm{SAB}(\mathrm{rs}, \mathrm{nd}) \mathrm{bc}$ & $\mathrm{SA}(\mathrm{rs}, \mathrm{nd}) \mathrm{b} \mathrm{sp}$ & II.1.0006 \\
\hline ESO 409-15 & $\mathrm{dIm}$ & $\mathrm{dIm}$ & II.1.0007 \\
\hline ESO 293-34 & S pec & S spw pec & II.1.0008 \\
\hline NGC 7 & $\mathrm{Sd} / \mathrm{Im} \mathrm{sp}$ & $\mathrm{Sd} \mathrm{sp}$ & II.1.0009 \\
\hline
\end{tabular}

a The galaxies are listed in order of J2000 R.A.; arrows indicate the beginning of an R.A. interval. See Table 1 for a summary of the meaning of CVRHS notations, and the notes to Table 6 for galaxies which have a different name in RC3. Figure numbers are either for paper I (I.1.nnn) or this paper (II.1.nnnn). Galaxies with an asterisk after the name are on $\mathrm{S}^{4} \mathrm{G}$ frames but are not part of the formal sample. Some of these are included in the atlas illustrations. If they are not, the name of the galaxy image where they can be found (in the publicly available database) is given.

(This table is available in its entirety in a machine-readable form.)

interpretation of some edge-on galaxies, but even so classification is still difficult for highly inclined galaxies.

\subsection{Application to the Full $S^{4} G$ Sample}

The classification of the full $\mathrm{S}^{4} \mathrm{G}$ sample was carried out by R. Buta in three phases: Phase 1, the initial examination of the full dataset as data were being collected; Phase 2, a reexamination of the full dataset made more than a year after data collection ended and without any reference to the Phase 1 results; and a partial (10\%) Phase 3 made 6 months after Phase 2 (also without reference to the previous phases) to better assess the internal consistency of the types derived by averaging the full Phase 1 and Phase 2 catalogs (Section 3.3). ${ }^{25}$

Table 2 provides the classifications from Phases 1 and 2 . (The Phase 3 classifications are listed in the Appendix.) A visual comparison shows generally good agreement between them, with differences appearing to be mostly random. Of the 2412 galaxies, the Phase 1 and 2 classifications are identical for 382 galaxies, or $16 \%$ of the sample. Restricting to the stage classification alone, the agreement is better, with $1370(57 \%)$ of the sample classified identically and $659(27 \%)$ differing by only \pm 1 step (e.g., as in Sab versus Sb), 167 (7\%) differing by \pm 2 steps (as in Sbc versus Scd), and 83 (3\%) differing by more than \pm 2 steps. Some of the cases of large disagreement are galaxies which appear to be of an early-type, yet have little or no bulge. These have classifications such as " $\mathrm{SB}(\mathrm{s}) 0 / \mathrm{a}[\mathrm{d}]$ ", where the "[d]" is meant to highlight the apparent lack of a bulge (alluding to the van den Bergh (1976) parallel sequence idea). This inconsistency could be real, but could also partly be a resolution effect in the sample.

For 1709 galaxies where it was possible to reliably assign family classifications, the Phase 1 and 2 classifications were identical for $1160(68 \%)$ of the sample, and differed by one classification interval (e.g., SB vs SAB for 264 (15\%), two intervals (e.g., SAB versus SB) for 264 (15\%), 3 intervals

\footnotetext{
${ }^{25}$ We thank the referee for suggesting this approach.
} 
(e.g., S $\mathrm{AB}$ versus $\mathrm{SB}$ for $6(0.4 \%)$, and the full range (e.g., SB versus $\mathrm{SA})$ for $15(0.9 \%)$ of the sample.

The classification and recognition of inner and outer ring and lens features can show variation, with some classifications in one phase not being noted in the other phase. In 14 cases in the catalog, the same feature has been classified as an inner ring or pseudoring in one phase, and as an outer ring or pseudoring in the other. As noted in Section 3.1, such ambiguities can occur especially for nonbarred or very weakly barred galaxies (Buta 1995). We adopt either the Phase 1 or Phase 2 classification for such cases, after a re-inspection of the image.

\subsection{Comparison of Classifications}

In this section, we use our independent classification phases to examine the internal agreement of CVRHS stage, family, and variety classifications of $\mathrm{S}^{4} \mathrm{G}$ galaxies. The stages and families are also compared with other sources.

For comparing classifications, and also for combining the Phase 1 and 2 catalogs, the letter classifications were coded with numbers (Table 3 ). For stages, the standard numerical $T$ index, which ranges from $T=-5$ for $\mathrm{E}$ galaxies to $T=+10$ for Im galaxies, was used as in RC3. For family classifications, following Baillard et al. (2011) we defined an index $F$ which ranges from 0.0 for $\mathrm{SA}$ galaxies to 1.0 for $\mathrm{SB}$ galaxies. We then set $F=0.25,0.50$, and 0.75 for $\mathrm{S} \underline{\mathrm{AB}}, \mathrm{SAB}$, and $\mathrm{SAB}$ galaxies, respectively. Similar codings were used for inner ring/ pseudoring/spiral and outer ring/pseudoring classifications. In Tables 3 and 4, these are referred to as the inner variety IV and outer variety $\mathrm{OV}$, respectively. Although the parentheses in VRHS classifications normally include only a single outer or inner feature (e.g., as in (R)SB(rs)ab), CVRHS classifications can include multiple inner and outer rings, lenses, or nuclear features. This complicates combining Phase 1 and 2 classifications for some of the galaxies, which had to be treated on an individual basis. For most of the galaxies, the assignment of a $T, F, \mathrm{IV}$, and $\mathrm{OV}$ index was straightforward. Nevertheless, we strongly emphasize that none of these numbers is a measured quantity; they are merely convenient codings for a given classification symbol. Table 4 shows how the Phase 1 and 2 $\langle T\rangle,\langle F\rangle,\langle\mathrm{IV}\rangle$, and $\langle\mathrm{OV}\rangle$ values convert into final classifications for most of the sample.

For some galaxies, the classification has two parts, as in " $\mathrm{SO}^{-}$ $\mathrm{sp} / \mathrm{E}(\mathrm{d}) 7$. This refers to an edge-on S0 showing a thin disk embedded within a disky (pointy-ended) E-like thick disk. (An example here is NGC 1032, shown in Figure 1.0193.) In such a case, only the first part determines the $T$-index, which in this example is -3 . Note that normal ellipticals are very rarely more flattened than E4 and genuine E galaxies are not necessarily found more flattened than E6 (van den Bergh 2009). The E(d) notation is from Kormendy \& Bender (1996), and was originally proposed for genuine elliptical galaxies. Two-part classifications recognizing a thick disk are only given for highly inclined galaxies.

\subsubsection{Stage and Family Phase 1, 2 Comparisons}

Figure 2 shows comparisons between the mean numerical type and family indices for the Phase 1 and 2 classifications for the full sample (top panels) and for the much smaller paper I subsample (middle panels). Filled circles show the mean $T$ and $F$ values for Phase 2 at each Phase 1 type, while the filled triangles show the mean $T$ and $F$ for Phase 1 at each Phase 2
Table 3

Numerical Codes

\begin{tabular}{|c|c|}
\hline Symbol & Numerical Index \\
\hline 1 & 2 \\
\hline Stages & $T$ \\
\hline $\mathrm{cE}$ & -6 \\
\hline E & -5 \\
\hline $\mathrm{E}^{+}$ & -4 \\
\hline $\mathrm{SO}^{-}$ & -3 \\
\hline $\mathrm{SO}^{\circ}$ & -2 \\
\hline $\mathrm{SO}^{+}$ & -1 \\
\hline $\mathrm{S} 0 / \mathrm{a}$ & 0 \\
\hline $\mathrm{Sa}$ & 1 \\
\hline $\mathrm{Sab}$ & 2 \\
\hline $\mathrm{Sb}$ & 3 \\
\hline $\mathrm{Sbc}$ & 4 \\
\hline $\mathrm{Sc}$ & 5 \\
\hline Scd & 6 \\
\hline $\mathrm{Sd}$ & 7 \\
\hline $\mathrm{Sdm}$ & 8 \\
\hline $\mathrm{Sm}$ & 9 \\
\hline $\operatorname{Im}$ & 10 \\
\hline $\mathrm{dE}, \mathrm{dS} 0, \mathrm{Sph}$ & 11 \\
\hline Families & $F$ \\
\hline SA & 0.00 \\
\hline 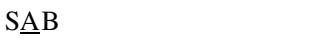 & 0.25 \\
\hline SAB & 0.50 \\
\hline SA $\underline{B}$ & 0.75 \\
\hline $\mathrm{SB}$ & 1.00 \\
\hline Inner and Outer Varieties & IV,OV \\
\hline (s), (no outer feature) & 0.00 \\
\hline$(\mathrm{rs})$ & 0.25 \\
\hline$(\mathrm{rs}),\left(\mathrm{R}^{\prime}\right)$ & 0.50 \\
\hline$(\underline{\mathrm{r}} \mathrm{s})$ & 0.75 \\
\hline$(\mathrm{r}),(\mathrm{R})$ & 1.00 \\
\hline$(\mathrm{r} \underline{1}),(\mathrm{RL})$ & 1.25 \\
\hline$(\mathrm{rl}),(\mathrm{RL})$ & 1.50 \\
\hline$(\underline{\mathrm{r}} \mathrm{l}),(\underline{\mathrm{R}} \mathrm{L})$ & 1.75 \\
\hline$(1),(\mathrm{L})$ & 2.00 \\
\hline
\end{tabular}

type. The differences, $\Delta T=T_{2}-T_{1}$ and $\Delta F=F_{2}-F_{1}$, can be used to judge how consistent the Phase 1 and 2 classifications are with respect to the refined divisions of CVRHS morphology.

The internal consistency of our Phase 1 and 2 stage and family classifications can be quantified using the numerical codings in Table 3 to calculate $\sigma_{12}(T)=\sigma(\Delta T)=\sqrt{\frac{\sum\left(T_{2}-T_{1}\right)^{2}}{N-1}}$ and $\sigma_{12}(F)=\sigma(\Delta F)=\sqrt{\frac{\Sigma\left(F_{2}-F_{1}\right)^{2}}{N-1}}$ for all galaxies in the sample having stage and family classifications in both phases. From these, we estimate the standard deviation of a single $T$ classification as $\sigma_{1}(T) \approx \sigma_{2}(T)=\sigma(T)=\sigma(\Delta T) / \sqrt{2}$ and of a single $F$ classification as $\sigma_{1}(F) \approx \sigma_{2}(F)=\sigma(F)=\sigma(\Delta F) / \sqrt{2}$. In a similar manner, when the Phase 1 and 2 classifications are averaged, we estimate the standard deviation of the mean types and families as $\sigma(<T>)=\sigma(\Delta T) / 2$ and $\sigma(<F>)=\sigma(\Delta F) / 2$, respectively.

The results of this analysis are compiled in Table 5, which gives these standard deviations in units of 1 stage interval for $T$ $(\Delta T=1.0)$ and 1 family interval for $F(\Delta F=0.25)$. For the samples in the top frames of Figure 2, the standard deviations between the phases are $\sigma(\Delta T)=1.04$ stage intervals and 
Table 4

Ranges for Combining Catalogs

\begin{tabular}{|c|c|}
\hline Symbol & Numerical Index Range \\
\hline 1 & 2 \\
\hline$\overline{(\mathrm{L})}$ & $1.875 \leqslant\langle\mathrm{OV}\rangle \leqslant 2.000$ \\
\hline$(\mathrm{R} \underline{\mathrm{L}})$ & $1.625 \leqslant\langle\mathrm{OV}\rangle<1.875$ \\
\hline$(\mathrm{RL})$ & $1.375 \leqslant\langle\mathrm{OV}\rangle<1.625$ \\
\hline$(\underline{\mathrm{RL}})$ & $1.125 \leqslant\langle\mathrm{OV}\rangle<1.375$ \\
\hline (R) & $0.750 \leqslant\langle\mathrm{OV}\rangle<1.125$ \\
\hline$\left(\mathrm{R}^{\prime}\right)$ & $0.250 \leqslant\langle\mathrm{OV}\rangle<0.750$ \\
\hline No outer feature & $0.000 \leqslant\langle\mathrm{OV}\rangle<0.250$ \\
\hline SA,IA & $0.00 \leqslant\langle F\rangle<0.15$ \\
\hline SㅁB,IAB & $0.15 \leqslant\langle F\rangle<0.35$ \\
\hline SAB,IAB & $0.35 \leqslant\langle F\rangle \leqslant 0.65$ \\
\hline SAB, IA $\underline{B}$ & $0.65<\langle F\rangle \leqslant 0.85$ \\
\hline $\mathrm{SB}, \mathrm{IB}$ & $0.85<\langle F\rangle \leqslant 1.00$ \\
\hline (s) & $0.00 \leqslant\langle\mathrm{IV}\rangle<0.15$ \\
\hline$(\underline{\mathrm{rs}})$ & $0.15 \leqslant\langle\mathrm{IV}\rangle<0.35$ \\
\hline (rs) & $0.35 \leqslant\langle\mathrm{IV}\rangle \leqslant 0.65$ \\
\hline$(\underline{\mathrm{r}} \mathrm{s})$ & $0.65<\langle\mathrm{IV}\rangle \leqslant 0.85$ \\
\hline$(\mathrm{r})$ & $0.85<\langle\mathrm{IV}\rangle \leqslant 1.15$ \\
\hline (1) & $1.85 \leqslant\langle\mathrm{IV}\rangle \leqslant 2.00$ \\
\hline (rl) & $1.65 \leqslant\langle\mathrm{IV}\rangle<1.85$ \\
\hline$(\mathrm{rl})$ & $1.35 \leqslant\langle\mathrm{IV}\rangle<1.65$ \\
\hline$(\underline{\mathrm{r}} \mathrm{l})$ & $1.15 \leqslant\langle\mathrm{IV}\rangle<1.35$ \\
\hline
\end{tabular}

$\sigma(\Delta F)=0.96$ of a family interval. These imply for a single estimate of $T$ an internal scatter of $\sigma(T)=0.74$ of a stage interval, and for a single estimate of $F$ an internal scatter of $\sigma(F)=0.68$ of a family interval. In both cases, the internal consistency of CVRHS classifications is slightly better than a single interval of the classification system. These results are supported by the partial Phase 3 analysis described in the Appendix.

\subsubsection{Inner and Outer Variety Phase 1, 2 Comparisons}

Figure 3 shows comparisons between the Phase 1 and 2 inner and outer variety classifications. These comparisons are more complicated than for stage and family because CVRHS classifications have more categories (such as lenses and ring/ pseudoring-lenses) compared to the VRHS system, and multiple features are recognizeable in some galaxies. Also, the classifications " $\left(\mathrm{r}^{\prime} 1\right)$ " and " $\left(\mathrm{R}^{\prime} \mathrm{L}\right)$ " do not fit well into the numerical codings in Table 3, and for the comparison, we have combined these categories with " $(\underline{r} \mathrm{~s})$ " and " $\left(\mathrm{R}^{\prime}\right)$," respectively, based on a mean stage analysis given in Figure 9 (Section 3.6). Because of these differences compared to stage and family, Figure 3 labels the axes using letter classifications rather than numerical codes.

For inner features, the comparison shows an increased scatter among lenses and ring-lenses, but nevertheless a good correlation between the Phase 1 and 2 classifications is found. Within the framework of the numerical codings in Table 3 , and for a numerical inner variety interval of 0.25 , the standard deviation between the Phase 1 and 2 inner variety classifications (Table 5) is $\sigma(\Delta \mathrm{IV})=\sqrt{\frac{\Sigma\left(I V_{2}-I V_{1}\right)^{2}}{N-1}}=1.16$ variety intervals (for $N=1486$ features in 1473 galaxies). A single estimate of variety thus has $\sigma(\mathrm{IV})=0.82$ of a variety interval, comparable to what was derived for stage and family.

The comparison of the Phase 1 and 2 outer variety classifications (Figure 3, right panel) uses a different numerical code from Table 3 because these do not account for the OLR categories $\mathrm{R}_{1}, \mathrm{R}_{1}{ }^{\prime}, \mathrm{R}_{2}{ }^{\prime}$, and $\mathrm{R}_{1} \mathrm{R}_{2}{ }^{\prime}$. Instead, the comparison is made in terms of 9 bins ranging from outer pseudorings $\left(\mathrm{R}^{\prime}\right)$ to outer lenses $(\mathrm{L})$. The ordering is based on the mean stage analysis in Figure 9. In terms of a numerical code ranging from 1 for category $\mathrm{R}^{\prime}$ to 9 for outer lenses, and for an outer variety interval of 1.0, the standard deviation of a single estimate of outer variety is $\sigma(\mathrm{OV})=1.27$ variety intervals (Table 5$)$. The partial Phase 3 analysis in the Appendix gives a similar result, but based on a much smaller subset of galaxies.

\subsubsection{Mean Classification Catalog}

The comparisons in Figure 2 and Figure 3 show good or at least reasonably good agreement between the Phase 1 and 2 classifications. For this reason, we do not favor one phase over the other and present in Table 6 unweighted averages of these classifications. In addition to the full average classification, the Table gives the average stage and family numerical indices. The average of the two phases was performed in the following manner.

Example:

NGC 4141:

Phase 1: $\mathrm{SB}(\mathrm{rs}) \mathrm{d}$ ( $\underline{\mathrm{s}}$ means the $\mathrm{s}$ is emphasized).

Phase 2: $\mathrm{SA} \underline{\mathrm{B}}(\mathrm{s}) \mathrm{dm}$ ( $\underline{\mathrm{B}}$ means the $\mathrm{B}$ is emphasized).

Table 6 average: $\mathrm{SB}(\mathrm{s}) \underline{\mathrm{dm}}$ ( $\underline{\mathrm{d}}$ means the $\mathrm{d}$ is emphasized).

The situation above occurs for $71 \%$ (1709) of the 2412 galaxies in the catalog. For $13 \%$ (326), the following type of situation occurs:

NGC 3044:

Phase 1: SB(s)dm sp

Phase 2: Sdm sp

Table 6 average: SB:(s:)dm sp,

where the colons are used to indicate that the family and variety are uncertain interpretations in this case. NGC 3044 is a nearly edge-on galaxy (hence the "sp"), and as we have already noted, it can still be difficult to interpret the structure of an edge-on galaxy, even in a dust-penetrated waveband.

As the above shows, the combination of the Phase 1 and 2 catalogs can lead to half-step stage classifications (e.g., Sd m). It was noted in Section 3.1.1 that underline stage notation like this is fully a part of VRHS galaxy classification, but for single estimates of types was used sparingly by de Vaucouleurs (1963). It was also used sparingly in Phase 1 classifications, and not at all in Phase 2 classifications. Table 5 shows that the mean stage classifications in Table 6 have $\sigma(\langle T\rangle)=0.52$ of a stage interval, essentially equal to one half-step. This implies that half step mean stages have only marginal significance, a conclusion supported by the partial Phase 3 analysis (Appendix). Nevertheless, we retain the underline notation in the mean stages in Table 6 in order to preserve as much information from the two phases as possible.

For the mean families in Table 6 , the Table 5 analysis gives $\sigma(\langle F\rangle)=0.48$ of a family interval, or half an underline interval. In this case, the de Vaucouleurs (1963) underline family classifications $\mathrm{SA}, \mathrm{S} \underline{\mathrm{B}}, \mathrm{SAB}, \mathrm{SA} \underline{\mathrm{B}}$, and $\mathrm{SB}$ have more significance than underline stages. The standard deviation of the mean inner varieties is $\sigma(<\mathrm{IV}>)=0.58$ 

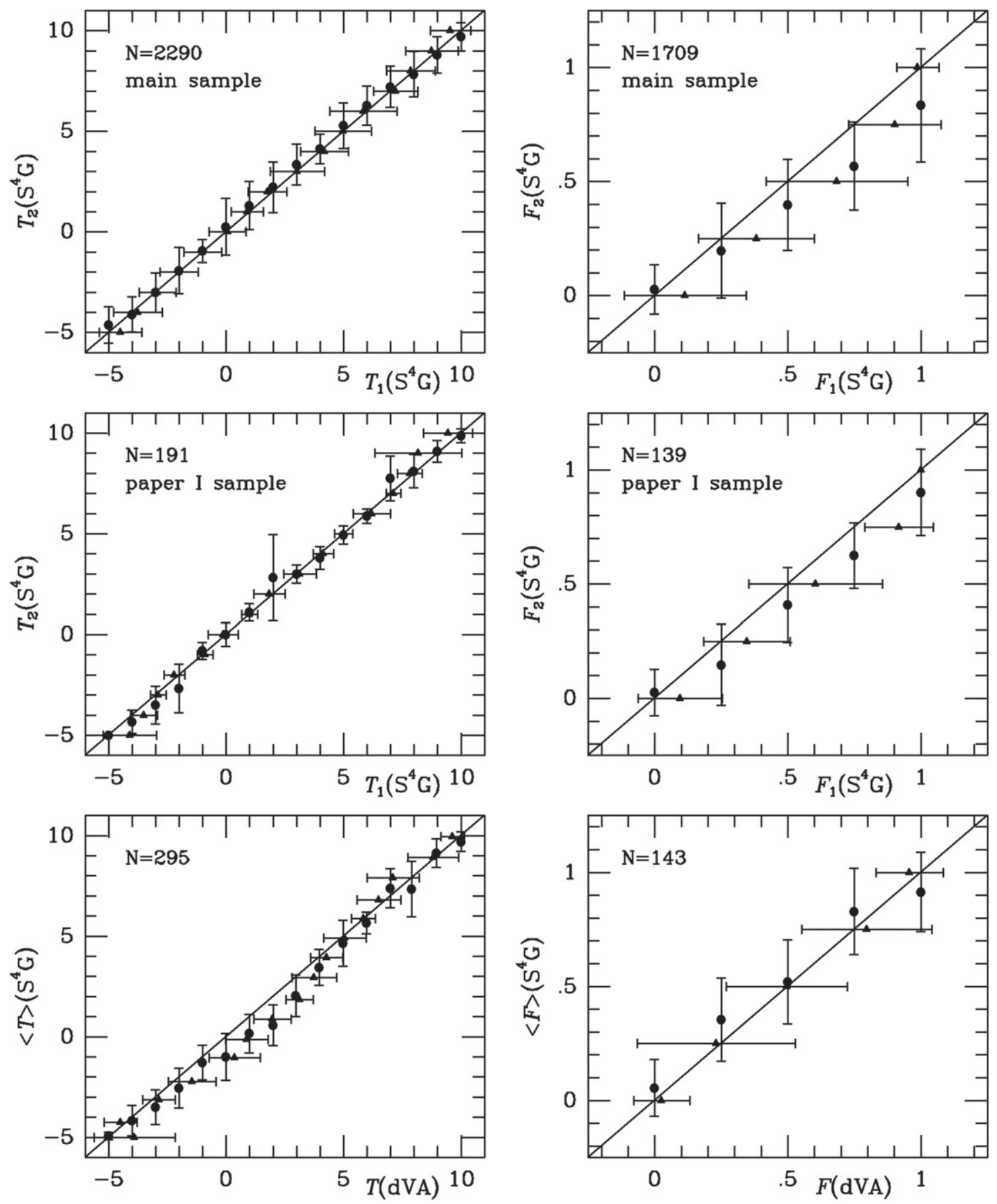

Figure 2. Top frames Comparisons between $\mathrm{S}^{4} \mathrm{G}$ phase 1 and phase 2 stages and families for all $\mathrm{S}^{4} \mathrm{G}$ galaxies for which both characteristics could be determined; middle frames Comparisons between $\mathrm{S}^{4} \mathrm{G}$ phase 1 and phase 2 stages and families for galaxies in the paper I sample only; bottom frames Comparison between $\mathrm{S}^{4} \mathrm{G}$ mean stages and families with the $B$ and $g$-band classifications for the same galaxies in the dVA. The error bars are $1 \sigma$ standard deviations in all frames.

of an inner variety interval while the standard deviation of mean outer varieties is $\sigma(\langle\mathrm{OV}\rangle)=0.90$ of an outer variety interval, also giving marginal significance to both classifications.

The mean classifications in Table 6 can be viewed as our "final" types because these have the benefit of two independent inspections which should average out most mistakes and misinterpretations. Nevertheless, the Phase 1 and 2 classifications listed separately in Table 2 still have value and are useful to highlight uncertain cases and to see how consistently a particular object has been interpreted.

\subsubsection{Other Comparisons}

The lower panels in Figure 2 compare the Table $6\langle T\rangle$ and $\langle F\rangle$ parameters with the $B$ - and $g$-band classifications in the dVA. In Paper I, we showed that mid-IR types generally agree well with $B$-band types, with a tendency for galaxies of $B$-band types S0/a to Sbc being classified slightly earlier in mid-IR type. This is the "earlier effect" in IR galaxy classification; it results from the decreased prominence of star-forming regions and the increased prominence of the bulge in IR images of $B$ band intermediate-type galaxies (Eskridge et al. 2002). The 
Table 5

Internal Consistency Analysis of CVRHS Classifications ${ }^{\mathrm{a}}$

\begin{tabular}{|c|c|c|}
\hline $\begin{array}{c}\text { Dimension } \\
1\end{array}$ & $\begin{array}{c}\text { Phase } 1,2 \\
2\end{array}$ & $\begin{array}{c}\text { Phase }<12>, 3 \\
3\end{array}$ \\
\hline \multicolumn{3}{|l|}{ Stage } \\
\hline Interval $^{\mathrm{b}}$ & 1.00 & 1.00 \\
\hline$\sigma(\Delta T)$ (intervals) & 1.04 & 0.86 \\
\hline$\sigma(T)$ (intervals) & 0.74 & 0.69 \\
\hline$\sigma(\langle T\rangle)$ (intervals) & 0.52 & $\ldots$ \\
\hline$N$ & 2290 & 238 \\
\hline \multicolumn{3}{|l|}{ Family } \\
\hline Interval $^{\mathrm{b}}$ & 0.25 & 0.25 \\
\hline$\sigma(\Delta F)$ (intervals) & 0.96 & 0.92 \\
\hline$\sigma(F)$ (intervals) & 0.68 & 0.79 \\
\hline$\sigma(<F>)$ (intervals) & 0.48 & $\ldots$ \\
\hline$N$ & 1709 & 196 \\
\hline \multicolumn{3}{|l|}{ Inner variety } \\
\hline Interval $^{\mathrm{b}}$ & 0.25 & 0.25 \\
\hline$\sigma(\Delta \mathrm{IV})$ (intervals) & 1.16 & 1.32 \\
\hline$\sigma(\mathrm{IV})$ (intervals) & 0.82 & 1.19 \\
\hline$\sigma(<\mathrm{IV}>)$ (intervals) & 0.58 & $\ldots$ \\
\hline$N$ & 1486 & 180 \\
\hline \multicolumn{3}{|l|}{ Outer variety } \\
\hline Interval $^{\mathrm{b}}$ & 1.00 & 1.00 \\
\hline$\sigma(\Delta \mathrm{OV})$ (intervals) & 1.80 & 1.57 \\
\hline$\sigma(\mathrm{OV})$ (intervals) & 1.27 & 1.29 \\
\hline$\sigma(<\mathrm{OV}>)$ (intervals) & 0.90 & $\ldots$ \\
\hline$N$ & 249 & 33 \\
\hline
\end{tabular}

${ }^{\text {a }}$ Col. (1): CVRHS classification dimension; (2) results of Phase 1 and 2 comparison. The parameters listed for stage are $\sigma(\Delta T)=\sigma_{12}(T)$, where $\Delta T=T_{2}-T_{1}$. Assuming Phase 1 and 2 are independent, then $\sigma_{1}=\sigma_{2}=\sigma(T)$ $=\sigma_{12} / \sqrt{(2) ; \sigma}(\langle T\rangle)=\sigma_{12} / 2$, where $\langle T\rangle$ is the value given in column 2 of Table 6; the same kinds of uncertainties are listed for the other classification dimensions; (3) results of Phase $310 \%$ experiment. In this column, $\Delta T=T_{3}-\langle T\rangle$ and $\sigma(T)=\sqrt{\sigma(\Delta T)^{2}-\sigma(\langle T\rangle)^{2}}$. In both columns 2 and 3, for stage and family, $N$ is the number of galaxies in the comparison, while for inner and outer variety, $N$ is the number of features.

${ }^{\mathrm{b}}$ Based on the numerical codings in Table 3, except for outer varieties, which use numbers from 1 to 9 for 9 types of features from $\mathrm{R}^{\prime}$ to (L).

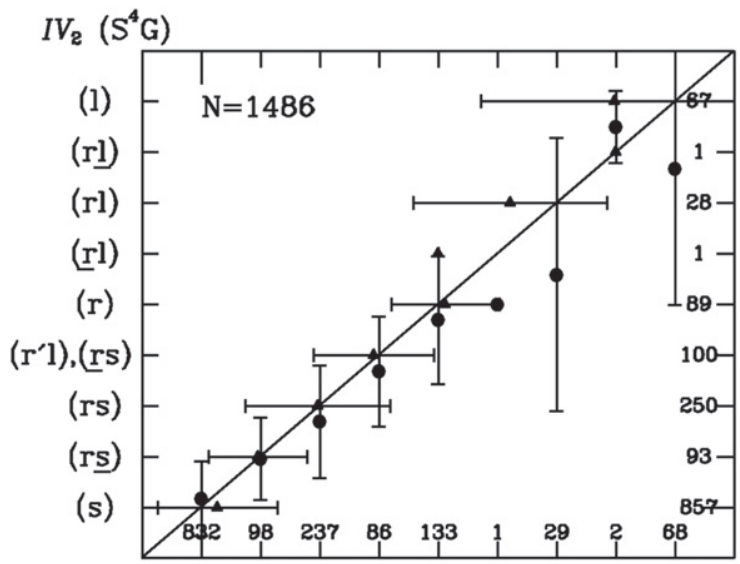

$(\mathrm{s})(\mathrm{rs})(\mathrm{rs}) \underset{\left(\mathrm{r}^{\prime} \mathrm{s}\right)}{(\mathrm{r})}(\mathrm{r})(\mathrm{rl})(\mathrm{rl})(\mathrm{rl})(\mathrm{l})$
Table 6

Mean CVRHS Classifications for $2412 \mathrm{~S}^{4} \mathrm{G}$ Galaxies ${ }^{\mathrm{a}}$

\begin{tabular}{|c|c|c|c|c|}
\hline Galaxy & $\langle\mathrm{T}\rangle$ & $\langle\mathrm{F}\rangle$ & $\langle$ Type $\rangle$ & Notes \\
\hline 1 & 2 & 3 & 4 & 5 \\
\hline \multicolumn{5}{|l|}{$\rightarrow$ R.A.: $0^{h} \leftarrow$} \\
\hline UGC 12893 & 11.0 & 0.00 & $\mathrm{dSA}(1) 0^{\circ} / \mathrm{Sph}$ & excellent case; face-on \\
\hline PGC 143 & 10.0 & $\cdots$ & $\mathrm{dIm}$ & $\begin{array}{l}\text { resolved dwarf; image } \\
\text { defects }\end{array}$ \\
\hline ESO 12-14 & 9.5 & 1.00 & $\mathrm{~S} / \mathrm{IB}(\mathrm{s}) \mathrm{m}$ & $\cdots$ \\
\hline UGC 17 & 10.0 & 0.50 & $\mathrm{IABm}$ & $\ldots$ \\
\hline NGC 7814 & 0.0 & 0.00 & SA0/a spw & excellent large edge-on \\
\hline NGC 7817 & 3.5 & 0.25 & $\begin{array}{l}\mathrm{S} \underline{\mathrm{A}} \mathrm{B}(\mathrm{rs}, \mathrm{nd}) \\
\underline{\mathrm{b}} \mathrm{csp}\end{array}$ & $\begin{array}{l}\text { grand-design spiral; } \\
\text { bright (nd); }\end{array}$ \\
\hline$"$ & $"$ & $"$ & $"$ & bar is end-on and barely \\
\hline$"$ & $"$ & $"$ & $"$ & visible; like N1365 \\
\hline ESO 409-15 & 10.0 & .. & dIAB:m & $\ldots$ \\
\hline ESO 293-34 & $\cdots$ & $\cdots$ & S spw pec & warped disk \\
\hline NGC 7 & 7.0 & $\cdots$ & Sd sp & large associations at ends \\
\hline$"$ & $"$ & $"$ & $"$ & of major axis \\
\hline NGC 14 & 10.0 & 0.75 & (L)IAB (s:)m & unusual for Im to have an \\
\hline$"$ & 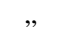 & 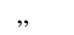 & $״$ & outer feature \\
\hline
\end{tabular}

${ }^{a}$ The galaxies are listed in order of J2000 R.A.; arrows indicate the beginning of a R.A. interval. See Tables 3 and 4 for the numerical codes and ranges used for combining the Phase 1 and 2 classifications. Arm classifications in the notes are from Table 9. Galaxies with an asterisk after the name are in $\mathrm{S}^{4} \mathrm{G}$ image frames but are not part of the formal sample.

(This table is available in its entirety in a machine-readable form.)

effect can be seen directly in the lower left panel of Figure 2, where the mean points tend to lie just below the line in this type range. There is also a fairly good correlation between $3.6 \mu \mathrm{m}$ families and dVA families.

Figure 4 (top panels) compares the mean $\mathrm{S}^{4} \mathrm{G}$ stage and family with the same classifications in RC3. The agreement on stages is similar to that for the dVA, showing the bending of points below the line in the type range $\mathrm{S} 0 / \mathrm{a}-\mathrm{Sbc}$. (This graph uses only galaxies having $|\Delta T| \leqslant 4.0$ stage intervals, in order to best show the systematic differences.) The comparison between $\mathrm{S}^{4} \mathrm{G}$ families and $\mathrm{RC} 3$ families has less resolution than

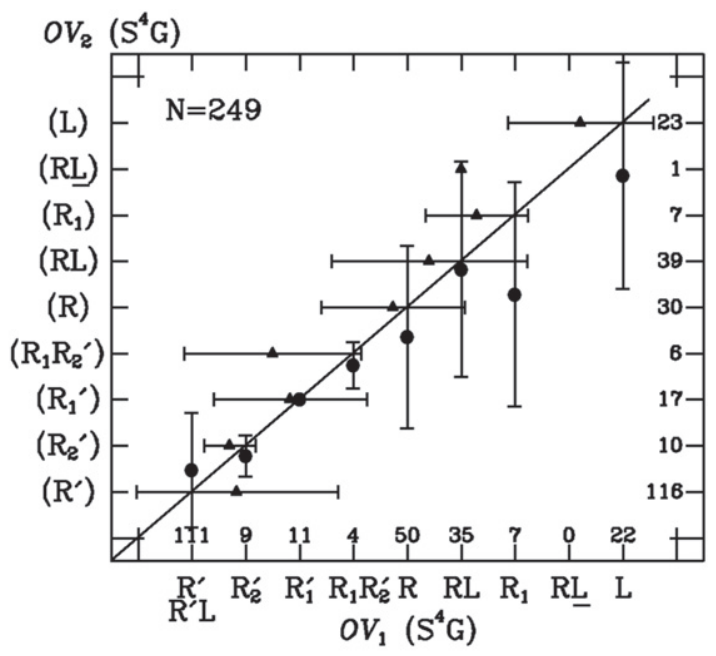

Figure 3. Comparison of Phase 1 and 2 inner and outer variety classifications of $S^{4} \mathrm{G}$ galaxies. The number $N$ in each panel is the number of features and includes multiple features in some cases. The number in each category is also given. 

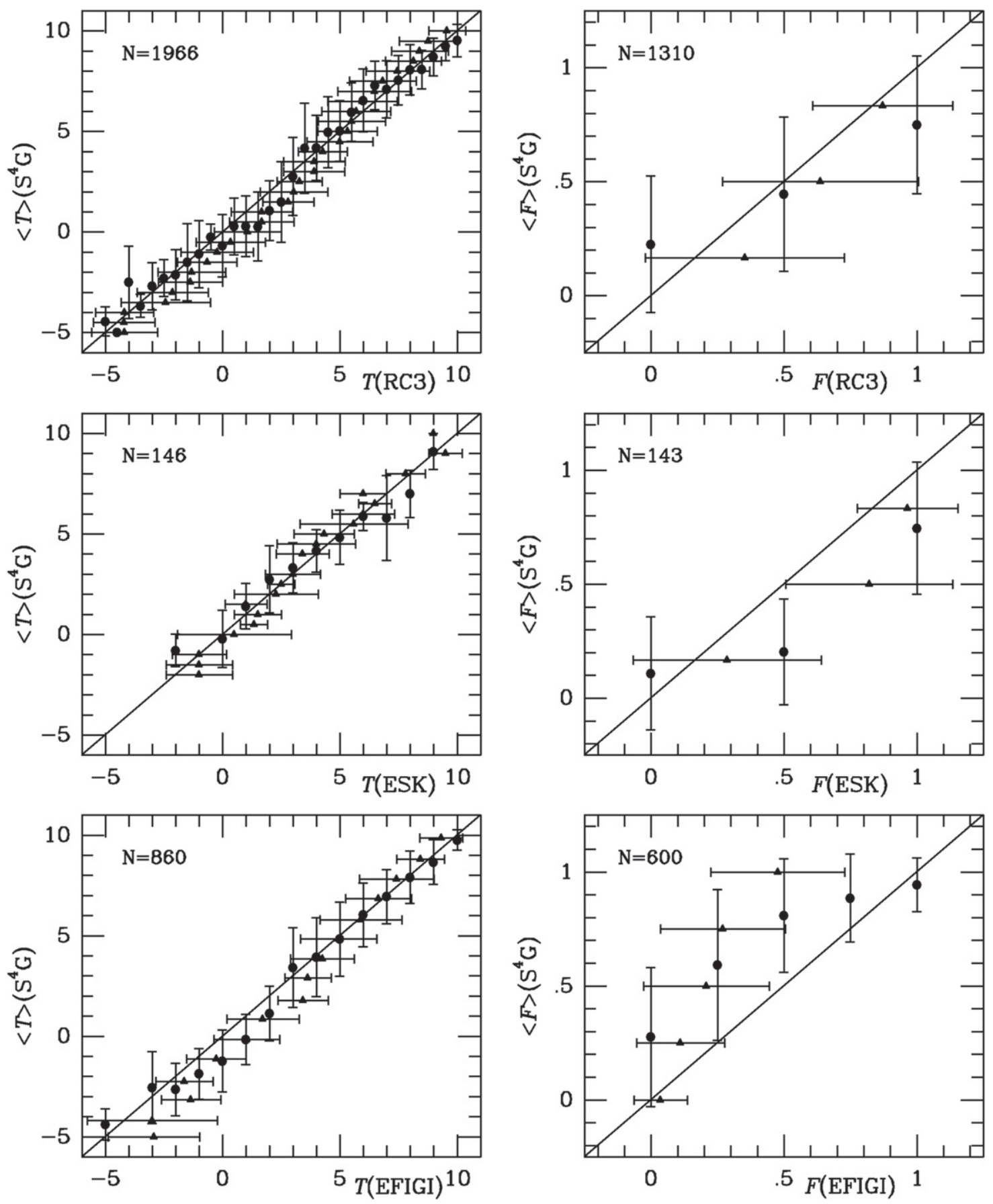

Figure 4. Top frames Comparisons between $\mathrm{S}^{4} \mathrm{G}$ mean stages and families and the $B$-band classifications for the same galaxies from RC3; middle frames The same graphs for the $H$-band classifications of Eskridge et al. (2002). Lower frames The same graphs for the optical SDSS classifications of Baillard et al. (2011, the "EFIGI" survey). The error bars are $1 \sigma$ standard deviations in all of the frames.

does that for the dVA, but a reasonable correlation is still found.

The Ohio State University Bright Spiral Galaxy Survey (OSUBSGS; Eskridge et al. 2002) was an optical/near-IR imaging survey of 205 nearby galaxies designed for a direct comparison between optical and near-IR galaxy classifications. The main optical filter used in the survey was the $B$ band and the main near-IR filter used was the $H$ band. $H$-band $(1.65 \mu \mathrm{m})$ images are similar to $3.6 \mu \mathrm{m}$ images in that the effects of extinction are greatly reduced and the light mostly traces the stellar mass. However, the Eskridge et al.
(2002) $H$-band images lack the depth of the $3.6 \mu \mathrm{m}$ IRAC images and are also less sensitive to star forming regions. The Eskridge et al. (2002) study demonstrated not only the "earlier" effect in near-IR galaxy classification, but also challenged previous suggestions that there was little correlation between optical and IR galaxy morphology (e.g., Block \& Puerari 1999).

Figure 4, middle frames shows a good correlation between the Eskridge et al. (2002) $\mathrm{H}$-band types and the Table 6 average $T$ values. The correlation of family classifications for the Eskridge et al. sample shows, in contrast, that galaxies 
classified as SA and SAB by Eskridge et al. (2002) are mostly classified as SABB in Table 6.

The lower panels compare $S^{4} \mathrm{G}$ mid-IR stages and families with the optical classifications from the "EFIGI" survey, where a team of 10 astronomers used SDSS images to classify 4458 nearby galaxies (Baillard et al. 2011). The lower left graph shows the comparison of stages, and like the comparisons with the dVA and RC3, the "earlier effect" is evident at intermediate stages. The comparison with EFIGI bar classifications shows an unusual pattern that is mainly due to methodology: the Baillard et al. family classifications are based on relative bar length, while $\mathrm{S}^{4} \mathrm{G}$ family classifications are based on apparent bar strength or contrast (as well as length). While there is some correlation, it appears $\mathrm{S}^{4} \mathrm{G}$ bar classifications are stronger on average than EFIGI bar classifications.

Our general conclusion from all of the comparisons described in this section is that CVRHS classifications have both good internal and external consistency. This consistency could, however, still mask resolution and especially inclination biases in the classifications, even if there were perfect agreement between phases or between different sources. Campbell et al. (2014) describe "imaging classification bias" in the context of the GalaxyZoo citizen morphological catalog, which they argue has excessive numbers of distant early-type galaxies that are likely to be misclassified spirals. As the authors correctly note, the ability to make reliable distinctions between galaxy types demands "the most stringent imaging requirements." Although $\mathrm{S}^{4} \mathrm{G}$ images are of much greater depth than groundbased near-IR images, the limited resolution coupled with high inclination could introduce some "imaging classification bias." This is explored further in the next sections.

\subsection{The Distribution of Mid-IR Galaxy Types}

For the purpose of examining the distribution of mid-IR galaxy types, families, and varieties, we restrict the analysis to the actual ("formal") sample of 2352 galaxies selected for the $\mathrm{S}^{4} \mathrm{G}$ (Section 2). The reason for this is that some of the extra galaxies that were on frames of formal sample galaxies are background objects beyond the survey's distance limit of $40 \mathrm{Mpc}$.

Figure 5 shows the distribution of these mean stages for the low inclination (low-i) and high inclination (high- $i$ ) galaxies in the $\mathrm{S}^{4} \mathrm{G}$ catalog. The distinction is made using the $3.6 \mu \mathrm{m}$ isophotal minor-to-major axis ratio, $q_{25.5}$, fitted at the (ABmagnitude) surface brightness level of $25.5 \mathrm{mag} \operatorname{arcsec}^{-2}$ (Munoz-Mateos et al. 2014). The low- $i$ sample is restricted to $q_{25.5} \geqslant 0.5$, corresponding to an inclination of $\approx 60^{\circ}$. The high- $i$ sample is restricted to $q_{25.5} \leqslant 0.5$. Spindle ("sp") galaxies in the catalog tend to have $q_{25.5} \leqslant 0.4$, and are mostly excluded from the low- $i$ sample. For both histograms in Figure 5, the $T$ values have been rounded to the nearest whole type.

The low- $i$ plot shows that nearly half of the formal $S^{4} \mathrm{G}$ sample consists of extreme late-type spiral and irregular galaxies of mid-IR stages Scd-Im. These constitute $48.5 \pm$ $1.4 \%$ of the 1240 low- $i$ formal sample galaxies having a mean stage. ${ }^{26}$ The right panel in Figure 5 shows, however, that this type range constitutes $66.0 \pm 1.4 \%$ of the high- $i$ sample. This

\footnotetext{
${ }^{26}$ For a percentage $p$ out of a number $N$ of sample objects, the error is calculated as $\Delta p(\%)=\sqrt{(100.0-p) p / N}$ (e.g., Laurikainen et al. 2013).
}

difference is likely to be partly due to the two-part manner in which we have classified some highly inclined galaxies (Section 4.4.2). Also, for highly inclined galaxies, mid-IR morphology often involves low contrast features and central concentrations viewed against a very bright background. These can lead to later-type classifications, and it is possible that some $\mathrm{Sc}$ galaxies are misclassified as $\mathrm{Sd}$ when the inclination is high. The problem can be exacerbated further if the image of a high inclination galaxy has relatively poor resolution.

The emphasis of the formal $\mathrm{S}^{4} \mathrm{G}$ sample on extreme late-type galaxies not only reflects its nature as a volume-limited sample, but also is due to the fact that the galaxies were selected on the basis of an HI radial velocity. The sample is rich in dwarf irregulars and bulgeless spirals, because these tend to be HIrich systems (e.g., Buta et al. 1994).

\subsection{The Distribution of Mid-IR Families}

The distribution of mean Phase 1 and 2 family classifications $(\langle F\rangle)$ is shown in Figure 6. The graphs are based only on formal sample galaxies where a family classification was recorded in both Phase 1 and Phase 2. Although bars are detectable in highly inclined galaxies (Section 4.3), for the purpose of examining the relative frequency of bars it is still best to restrict the analysis to the low- $i$ subsample. Also, the graphs are plotted after combining $\mathrm{SA}$ and $\mathrm{SAB}$ into $\mathrm{SA}$, and SA $\underline{B}$ and $\mathrm{SB}$ into SB, since the underline categories are always under-represented compared to the main categories.

The barred family classification fraction, $f(F \geqslant 0.5)$, is defined as

$$
f(F \geqslant 0.5)=\frac{N(\mathrm{SAB})+N(\mathrm{SAB})+N(\mathrm{SAB})}{N}
$$

where $N$ is the total number of galaxies in the sample that can be subdivided in this manner (i.e., $N=N(\mathrm{SA})+N(\mathrm{SAB})+N$ $(\mathrm{SAB})+N(\mathrm{SAB})+N(\mathrm{SB}))$ and the counts are based on the mean letter-classifications in Table 6, not the $\langle F\rangle$ index. This assumes that $\mathrm{SAB}$ galaxies have obvious bars, even if these bars might be less striking than those seen in a classical SB galaxy. We anticipate that a sample like the $\mathrm{S}^{4} \mathrm{G}$ might give a reliable assessment of this fraction, because no bars would be overlooked due to star formation or excessive internal extinction, two problems which complicate $B$-band visual bar fractions (Eskridge et al. 2000).

Figure 6 shows estimates of $f(F \geqslant 0.5)$ for the 1059 low- $i$ formal $\mathrm{S}^{4} \mathrm{G}$ galaxies having Phase 1 and 2 family classifications, and for subsets of low- $i$ galaxies separated by stage. Over the type range $\mathrm{S}^{-}$to $\operatorname{Im}, f(F \geqslant 0.5)$ is about $2 / 3$, similar to previous studies such as de Vaucouleurs (1963), Eskridge et al. (2000), and Marinova \& Jogee (2007), although the latter two studies are based on the OSUBSG (Eskridge et al. 2002) which did not include very many galaxies having RC3 stages later than Scd.

When divided by type, the histograms are distinctly different: for Scd-Sm galaxies, the SB bin has the highest number of galaxies, while for $\mathrm{S} 0 / \mathrm{a}-\mathrm{Sc}$ galaxies, the SA bin has the highest number. As a result, the barred family classification fractions are significantly different: $f(F \geqslant 0.5)=81.0 \pm 2.0 \%$ for Scd-Sm galaxies and $55.0 \pm 2.2 \%$ for $\mathrm{S} 0 / \mathrm{a}-\mathrm{Sc}$ galaxies.

Further insight into these histograms is provided in Figure 7, left, which shows $f(F \geqslant 0.5)$ as a function of individual stages from S0/a to Im, again restricted to the low- $i$ sample. Much of the difference between the early and late samples is due to what appears to be a substantial drop in $f$ at stages $\mathrm{S} \underline{\mathrm{b}} \mathrm{c}$ to Sc. As a 

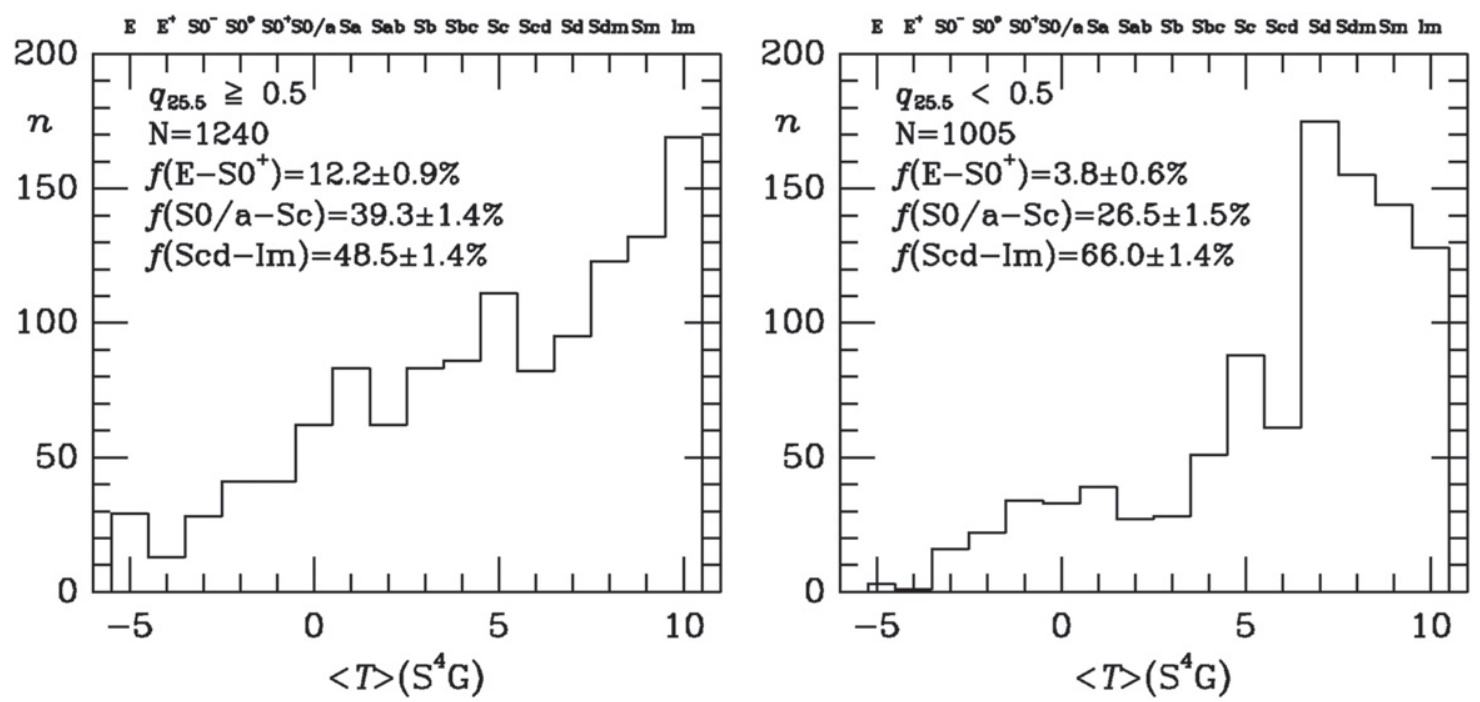

Figure 5. Distribution of $\mathrm{S}^{4} \mathrm{G}$ galaxies vs. mean mid-IR type index (Table 6, column 2) for 2245 formal sample galaxies divided according to the $3.6 \mu \mathrm{m}$ isophotal axis ratio, $q_{25.5}$, measured at the (AB) magnitude surface brightness level of 25.5 mag arcsec ${ }^{-2}$. The "low- $i$ " sample is at left and the "high- $i$ " sample is at right. The fractions of galaxies out of the total numbers $N$ in different type ranges are indicated. The distributions are plotted in unit stage intervals, with half-step averages rounded to the nearest full type.
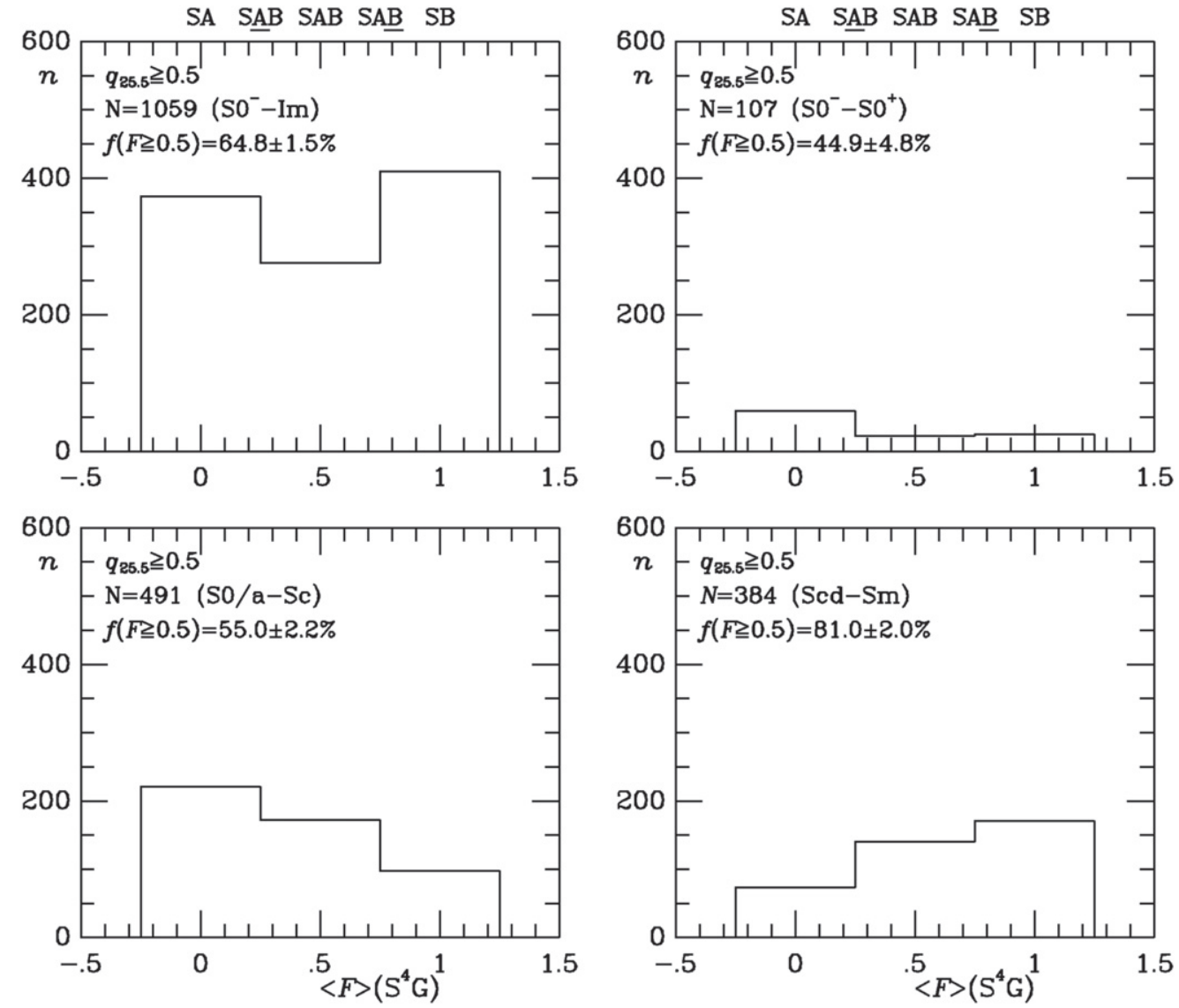

Figure 6. Top left the distribution of $S^{4} \mathrm{G}$ galaxies vs. mean mid-IR family index (Table 6, column 3 ) for 1059 "low-i" sample galaxies where a family classification was recorded in both Phase 1 and Phase 2. The other frames show distributions for subsets of this sample divided according to mid-IR stage. The fractions, $f$, of SAB, $\mathrm{SAB}$, and SB galaxies out of the total numbers $N$ listed are indicated. The distributions are plotted after combining SA and SAB into the SA bin and SB and SA $\underline{B}$ into SB.

check on this, Figure 7, right, shows the same graph for 866 of the same galaxies using $\mathrm{RC} 3$ classifications. The large drop in $f$ for mid-IR classification is much less evident when $B$-band RC3 classifications are used. The drop in the mid-IR fractions occurs in the type domain where the "earlier effect" begins to be noticeable. In spite of the drop, both graphs support a higher barred family classification fraction for galaxies of types Scd and later. 

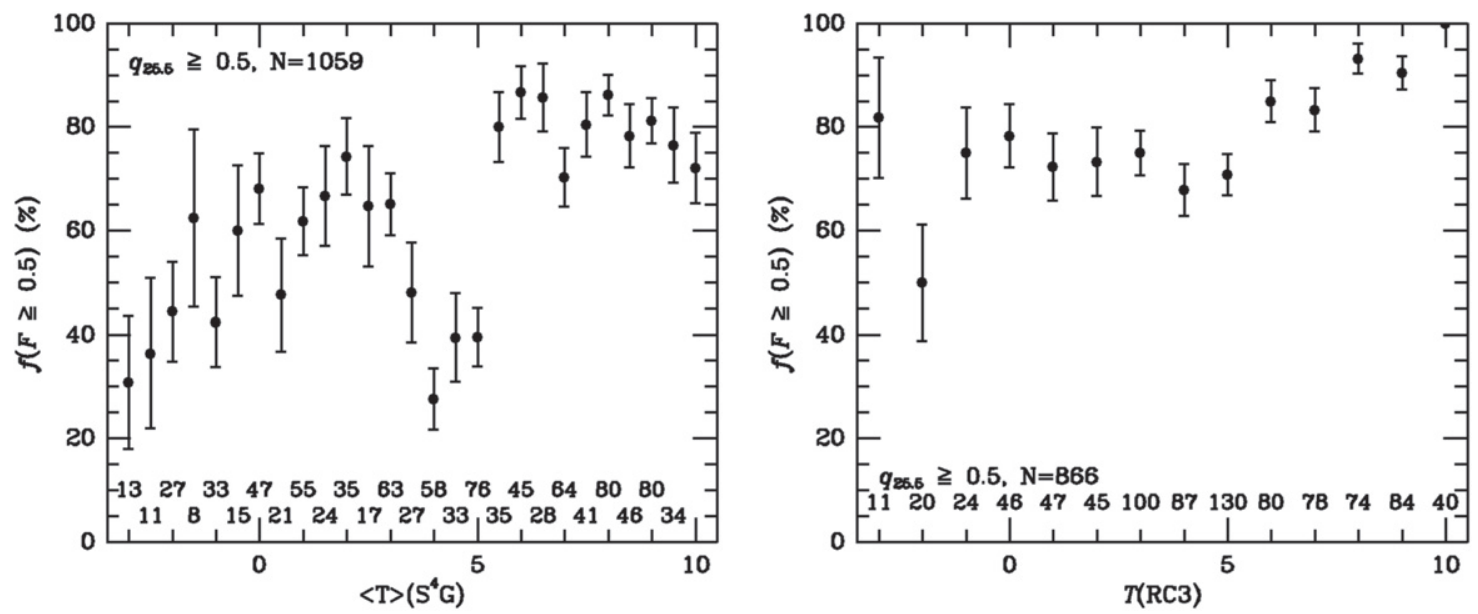

Figure 7. (Left): graph of the mid-IR barred family classification fraction as a function of mean mid-IR stage (Table 6, column 2 ) for 1059 galaxies in the low- $i \mathrm{~S}^{4} \mathrm{G}$ subsample. (Right): graph of the $B$-band barred classification fraction $(\mathrm{SAB}+\mathrm{SB})$ as a function of stage for 866 of the same galaxies as in the left graph, based on RC3 classifications. Error bars are derived as $\Delta p(\%)=\sqrt{(100.0-p) p / n}$, where $p$ is the fraction and $n$ is the number of galaxies at each stage (indicated on each graph).

Table 7 lists the dependence of $f$ in the early-type, late-type, and full spiral subsamples as a function of $q_{\min }$, the minimum mid-IR isophotal axis ratio for the sample. (The "low-i" sample we have been using corresponds to $\left.q_{\min }=0.5\right)$. The table shows that the significant difference between the early- and late-type samples diminishes but is not completely eliminated except for the most face-on subsample $\left(q_{\min }=0.9\right)$.

These results are actually fairly consistent with previous studies. For example, Barazza et al. (2008) used ellipse fits to infer the presence of bars in a large sample of nearby SDSS galaxies over the absolute magnitude range $-18.5 \leqslant M_{B} \leqslant$ -22.0 , and concluded that $87 \%$ of bulgeless disk galaxies are barred as opposed to $44 \%$ of bulge+disk galaxies. Galaxies of CVRHS types Scd-Sm not only do not have classical bulges, they also generally do not have pseudobulges. Barazza et al. also found that the optical bar fraction rises with decreasing total galaxy mass, a result which is consistent with the massdependent bimodality of the bar fraction noted by Nair \& Abraham (2010). Scd-Sm galaxies tend to be less luminous on average than S0/a-Sc galaxies (e.g., the dVA), and therefore are likely less massive than $\mathrm{S} 0 / \mathrm{a}-\mathrm{Sc}$ galaxies.

The upper right panel of Figure 6 shows the distribution of family classifications for 107 So galaxies in the low- $i$ subsample. The most common family classification for $\mathrm{S}^{4} \mathrm{G}$ S0 galaxies is SA, and the barred family fraction is $45 \pm 5 \%$. This smaller fraction of bars is also consistent with previous studies (Aguerri et al. 2009; Laurikainen et al. 2009; Buta et al. 2010a, 2010b), but a more reliable assessment must await the $\mathrm{S}^{4} \mathrm{G}$ extension.

Note that the "barred family classification fraction" is not necessarily the same as the cosmologically significant "bar fraction" (e.g., Sheth et al. 2008, 2014; Swardt et al. 2015). This is because the types of bars seen in nearby latetype galaxies may not necessarily be the ones we see at high redshift. The distinction is discussed further in Section 4.3.3.

\subsection{Inner and Outer Varieties}

In this section we examine some of the systematics of variety classifications in the $\mathrm{S}^{4} \mathrm{G}$ catalog. Inner varieties range from the pure (s) shape to inner pseudorings ( $r s$ ) to inner rings (r) and finally to inner lenses (1). Outer varieties include no feature for an unclosed spiral, outer pseudorings $\left(\mathrm{R}^{\prime}\right)$, outer rings $(\mathrm{R})$,
Table 7

$\mathrm{S}^{4} \mathrm{G}$ Bar Classification Fraction ${ }^{\mathrm{a}}$

\begin{tabular}{ccrcccr}
\hline \hline$q_{\min }$ & $\begin{array}{c}f_{e}(F \geqslant 0.5) \\
(\mathrm{S} 0 / \mathrm{a}-\mathrm{Sc})\end{array}$ & $N_{e}$ & $\begin{array}{c}f_{l}(F \geqslant 0.5) \\
\text { Scd-Sm }\end{array}$ & $N_{l}$ & $\begin{array}{c}f(F \geqslant 0.5) \\
\text { S0/a-Sm }\end{array}$ & $N$ \\
1 & \multicolumn{1}{c}{2} & \multicolumn{1}{c}{3} & 4 & 5 & 6 & 7 \\
\hline 0.1 & $55.8 \pm 1.9$ & 694 & $84.9 \pm 1.3$ & 720 & $70.6 \pm 1.2$ & 1414 \\
0.2 & $55.7 \pm 1.9$ & 691 & $84.2 \pm 1.4$ & 689 & $69.9 \pm 1.2$ & 1380 \\
0.3 & $55.7 \pm 1.9$ & 659 & $82.7 \pm 1.6$ & 584 & $68.4 \pm 1.3$ & 1243 \\
0.4 & $55.2 \pm 2.1$ & 585 & $82.2 \pm 1.8$ & 466 & $67.2 \pm 1.4$ & 1051 \\
0.5 & $55.0 \pm 2.2$ & 491 & $81.0 \pm 2.0$ & 384 & $66.4 \pm 1.6$ & 875 \\
0.6 & $57.7 \pm 2.5$ & 378 & $78.0 \pm 2.4$ & 291 & $66.5 \pm 1.8$ & 669 \\
0.7 & $58.5 \pm 3.0$ & 265 & $73.7 \pm 3.2$ & 194 & $64.9 \pm 2.2$ & 459 \\
0.8 & $54.8 \pm 4.0$ & 157 & $71.3 \pm 4.2$ & 115 & $61.8 \pm 2.9$ & 272 \\
0.9 & $67.9 \pm 6.4$ & 53 & $76.5 \pm 7.3$ & 34 & $71.3 \pm 4.9$ & 87 \\
\hline
\end{tabular}

${ }^{\text {a }}$ Col. (1) minimum isophotal axis ratio at $\mu_{\mathrm{AB}}(3.6 \mu \mathrm{m})=25.5 \mathrm{mag} \operatorname{arcsec}^{-2}$; (2) fraction (percent) of classified bars in galaxies having isophotal axis ratio $q_{25.5} \geqslant q_{\min }$ in the earlier-type range $\mathrm{S} 0 / \mathrm{a}-\mathrm{Sc}$; (3) No. of galaxies in the $\mathrm{S} 0 / \mathrm{a}$ - Sc subsample; $(4,5)$ same as $(2,3)$ for the later-type sample (Scd-Sm); $(6$, $7)$ same as $(2,3)$ for the combined samples.

outer ring-lenses (RL), outer lenses (L), and OLR subclass features $R_{1}, R_{1}{ }^{\prime}, R_{2}{ }^{\prime}$, and $R_{1} R_{2}{ }^{\prime}$.

Figure 8 shows the inner ring-lens fraction as a function of mid-IR stage. The fraction strongly declines with advancing stage from early to late. Although S0s can and do appear in (s) variety form, this is far less abundant than ring and lens varieties. Comparison of Figure 8 with Figure 7 shows that where the barred classification fraction is highest, the fraction of inner rings and lenses is lowest. This appears to imply that gas-rich galaxies are less capable of forming lenses or the kinds of rings that are typically seen in early-type barred and nonbarred galaxies.

Similar to Table 7, Table 8 shows the inner ring/pseudoring fraction for $\mathrm{S} 0 / \mathrm{a}-\mathrm{Sc}, \mathrm{Scd}-\mathrm{Sm}$, and $\mathrm{S} 0 / \mathrm{a}-\mathrm{Sm}$ galaxies as a function of the value of $q_{\min }$ used to define the subsamples. These features are found in $\approx 50 \%$ of S0/a-Sc galaxies and $\approx 13 \%$ of Scd-Sm galaxies. Across the whole spiral sequence, about $1 / 3$ of the galaxies has an inner ring/pseudoring.

Figure 9 (top left) shows how both mean stage and mean family correlate with inner variety classifications. A strong correlation between mean stage and variety is seen in the sense 


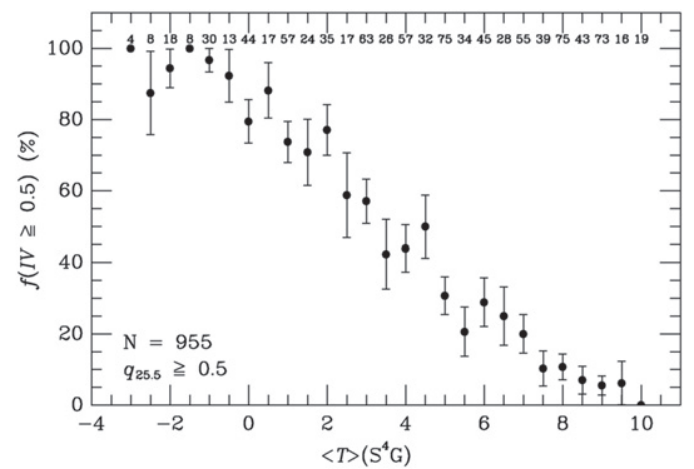

Figure 8. Graph of the inner ring/lens classification fraction (rs, rs, r, rl, and 1) as a function of mean mid-IR stage (Table 6, column 2) for 955 galaxies from the low- $i \mathrm{~S}^{4} \mathrm{G}$ subsample. The number of galaxies at each stage is indicated. Error bars calculated in the same manner as for Figure 7.

that (s)-shaped spirals are on average mid-IR stage Scd, while (r)-variety spirals average at mid-IR stage Sa. Ring-lenses average at stages $\mathrm{SO}^{+}$to $\mathrm{S} 0 / \mathrm{a}$. The upper right panel of Figure 9 shows the average visual bar strength for (s), (rs), and (rs) varieties is a little higher than for $(\underline{r} \mathrm{~s}),(\mathrm{r}),(\mathrm{rl})$, and (l) varieties.

Similar results are found for the outer variety features (Figure 9, lower left). Galaxies having outer pseudorings average between stages $\mathrm{Sb}$ and $\mathrm{Sbc}$, while closed outer rings are found on average in S0/a and Sa galaxies. Outer ring-lenses (RL), OLR subclass rings $\mathrm{R}_{1}$, and outer lenses (L) are found on average in $\mathrm{S}^{+}$to $\mathrm{S} 0 /$ a galaxies. The lower right frame of Figure 9 shows that average visual bar strength for outer pseudorings is only slightly higher than for outer rings and ring-lenses. In the NIRSOS sample of bright early-type disk galaxies (Laurikainen et al. 2011), outer lenses are common in the non-barred S0s. Some of these are missing from $S^{4} G$ because of the sample selection, which picked up only the fairly gas rich galaxies.

\section{MORPHOLOGICAL HIGHLIGHTS OF THE CATALOG}

In this section, we examine some of the highlights of mid-IR galaxy morphology. The "stellar structures" we discuss are mostly aspects of normal galaxies rather than strongly interacting or merging galaxies. Stellar rings, characteristics of early- and late-type bars, dust-penetrated views of classical edge-on galaxies, unusual spirals, the possible connection between late-type and spheroidal galaxies, and other special cases of interest are what we focus on here. These complement the highlights described in Paper I. All of the galaxies we illustrate in this section were selected for special attention from the large database because they are considered to be good examples of the morpholological characteristics that we have chosen to highlight.

\subsection{Mid-IR CVRHS Sequence}

The first major conclusion one can draw from examining the mid-IR morphologies of a large sample of galaxies is that the stellar mass structure of galaxies is not hidden too deeply by dust in optical imaging. The montages in Figures 10 and 11 show the same kinds of morphologies as are seen in blue light, ordered in a three-pronged "tuning fork" by VRHS family (SA, $\mathrm{SAB}, \mathrm{SB}$ ) along the standard VRHS sequence of stages.
Table 8

$\mathrm{S}^{4} \mathrm{G}$ Inner Ring/Pseudoring Fraction ${ }^{\mathrm{a}}$

\begin{tabular}{|c|c|c|c|c|c|c|}
\hline$q_{\min }$ & $\begin{array}{c}v_{e}(\mathrm{rs}, \underline{\mathrm{rs}}, \mathrm{r}) \\
(\mathrm{S} 0 / \mathrm{a}-\mathrm{Sc})\end{array}$ & $N_{e}$ & $\begin{array}{c}v_{l}(\mathrm{rs}, \underline{\mathrm{rs}}, \mathrm{r}) \\
\mathrm{Scd}-\mathrm{Sm}\end{array}$ & $N_{l}$ & $\begin{array}{c}v(\mathrm{rs}, \underline{\mathrm{rs}}, \mathrm{r}) \\
\mathrm{S} 0 / \mathrm{a}-\mathrm{Sm}\end{array}$ & $N$ \\
\hline 1 & 2 & 3 & 4 & 5 & 6 & 7 \\
\hline 0.1 & $48.6 \pm 2.0$ & 621 & $9.6 \pm 1.2$ & 634 & $28.9 \pm 1.3$ & 1255 \\
\hline 0.2 & $48.8 \pm 2.0$ & 619 & $9.9 \pm 1.2$ & 614 & $29.4 \pm 1.3$ & 1233 \\
\hline 0.3 & $49.6 \pm 2.1$ & 591 & $11.2 \pm 1.4$ & 528 & $31.5 \pm 1.4$ & 1119 \\
\hline 0.4 & $51.0 \pm 2.2$ & 524 & $13.0 \pm 1.6$ & 431 & $33.8 \pm 1.5$ & 955 \\
\hline 0.5 & $51.1 \pm 2.4$ & 444 & $13.0 \pm 1.8$ & 354 & $34.2 \pm 1.7$ & 798 \\
\hline 0.6 & $51.3 \pm 2.7$ & 343 & $13.1 \pm 2.0$ & 274 & $34.4 \pm 1.9$ & 617 \\
\hline 0.7 & $52.7 \pm 3.2$ & 243 & $14.3 \pm 2.6$ & 182 & $36.2 \pm 2.3$ & 425 \\
\hline 0.8 & $50.4 \pm 4.2$ & 141 & $15.0 \pm 3.4$ & 107 & $35.1 \pm 3.0$ & 248 \\
\hline 0.9 & $52.1 \pm 7.2$ & 48 & $24.2 \pm 7.5$ & 33 & $40.7 \pm 5.5$ & 81 \\
\hline
\end{tabular}

${ }^{\text {a }}$ Col. (1) minimum isophotal axis ratio at $\mu_{\mathrm{AB}}(3.6 \mu \mathrm{m})=25.5 \mathrm{mag} \operatorname{arcsec}^{-2}$;

(2) fraction (percent) of classified inner rings and pseudorings in galaxies having isophotal axis ratio $q_{25.5} \geqslant q_{\min }$ in the earlier-type range S0/a - Sc; (3) no. of galaxies in the S0/a-Sc subsample; $(4,5)$ same as $(2,3)$ for the later-type sample $(\mathrm{Scd}-\mathrm{Sm}) ;(6,7)$ same as $(2,3)$ for the combined samples.

$\mathrm{S} 0$ galaxies are subdivided into stages $\mathrm{S}^{-}, \mathrm{S}^{\circ}$, and $\mathrm{S}^{+}$in a sequence of increasing structure (but not necessarily decreasing bulge-to-total luminosity ratio). In $\mathrm{S}^{-}$galaxies, bars and lenses are usually fairly subtle, and the disk is virtually featureless. $\mathrm{S}^{\circ}$ galaxies have more obvious lenses, ring-lenses, and bars. The most structured S0s are stage $\mathrm{SO}^{+}$, which is the type where rings (without spirals) are most prominent (Section 4.2). In S0/a galaxies, the rings may break into tight spiral structure.

The spiral and irregular sequences also show well-defined and representative types. The decrease in average bulge-to-total luminosity ratio is evident. In general, the same three criteria Hubble used for spirals: the relative prominence of the bulge, the degree of resolution of the arms, and the degree of openness of the arms, are still applicable in the mid-IR, as already noted in paper I.

Several of the galaxies in Figure 11, if displayed in blue light, would appear in a different cell. For example, NGC 7479 is classified as stage $\mathrm{Sc}$ in the $B$ band but as stage $\mathrm{Sb}$ in the mid-IR, while NGC 4548 is classified as stage $\mathrm{Sb}$ in the $B$ band and $\mathrm{Sa}$ in the mid-IR. This is the "earlier" effect noted in Section 3.3.

\subsection{The Mid-IR Morphology of Galactic Rings and Lenses}

Rings, pseudorings, and lenses are important features of disk-shaped galaxies. Rings are often sites of active star formation and come in several different types: nuclear, inner, and outer, that occur on very different linear scales. Rings and lenses have distinctive shapes and orientations that tie them to aspects of internal dynamics (Buta \& Combes 1996). Generally highly flattened, rings and lenses are most commonly seen in early-type disk galaxies and are likely some of the clearest products of secular evolution that we can observe in galaxies (Knapen 2010).

Recognizing all of the variations of the stellar mass morphology of galactic rings and lenses can add considerable complexity between the brackets of CVRHS $3.6 \mu \mathrm{m}$ galaxy classifications (Table 1). In this section, we examine this complexity, beginning with CVRHS stage $\mathrm{SO}^{+}$, the first stage where all of these phenomena become prominent, and then 


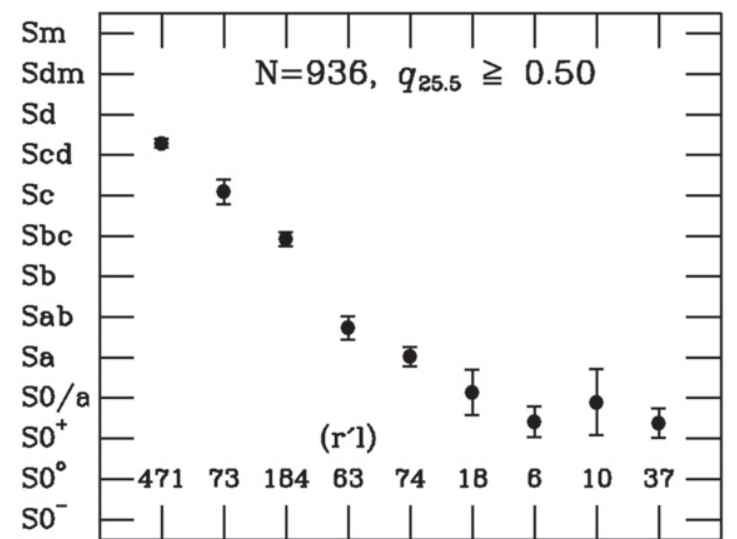

(s) $(\mathrm{rs})(\mathrm{rs})(\underline{\mathrm{rs}})(\mathrm{r})(\underline{\mathrm{r}} \mathrm{)})(\mathrm{rl})(\underline{\mathrm{rl}})(\mathrm{l})$

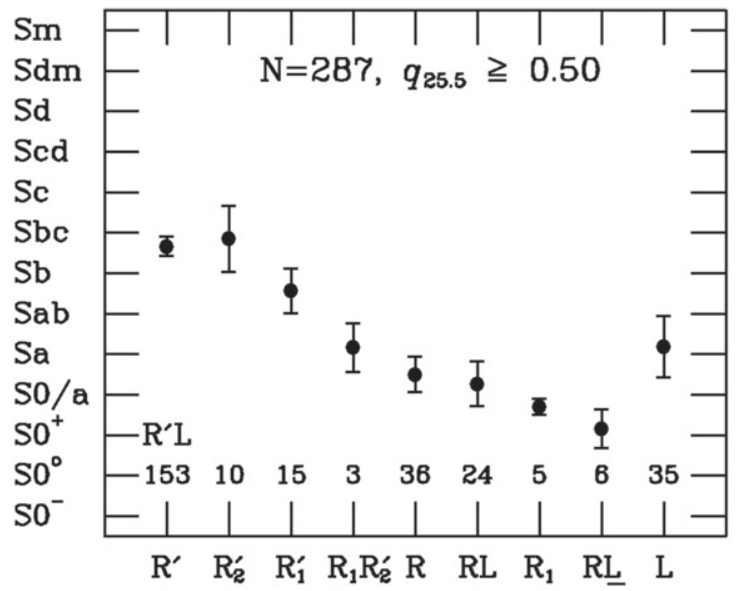

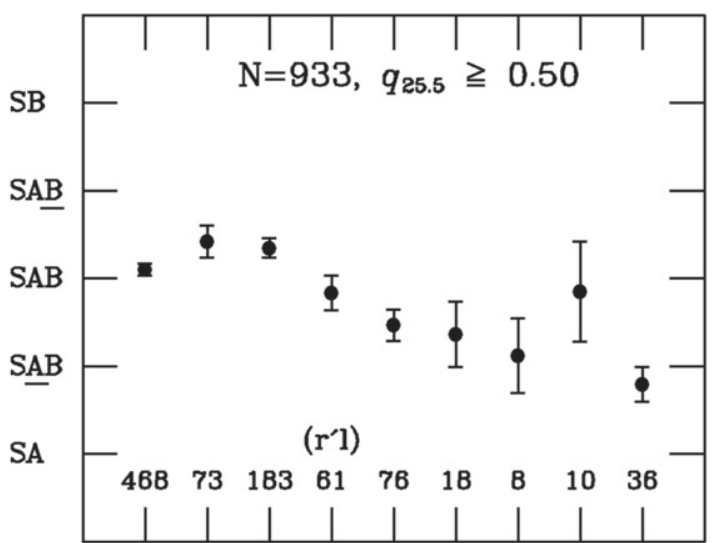

(s) (rs) (rs) (rs) (r) (rl) (rl) (rl) (l)

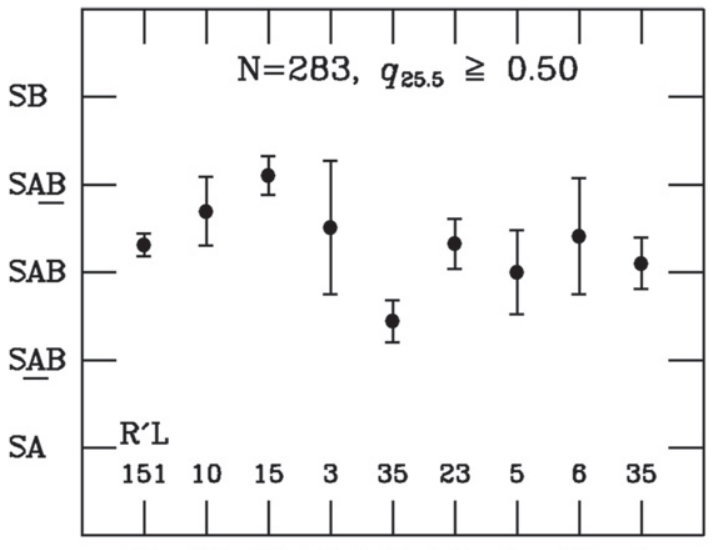

$\begin{array}{llllllllll}\mathrm{R}^{\prime} & \mathrm{R}_{2}^{\prime} & \mathrm{R}_{1}^{\prime} & \mathrm{R}_{1} \mathrm{R}_{2}^{\prime} \mathrm{R} & \mathrm{RL} & \mathrm{R}_{1} & \mathrm{RL} & \mathrm{L}\end{array}$

Figure 9. Graphs of mean mid-IR stage and family vs. inner and outer variety classifications. In these graphs, $r^{\prime} 1$ is combined with $\underline{r} s$ and $\mathrm{R}^{\prime} \mathrm{L}$ is combined with $\mathrm{R}^{\prime}$. The number $n$ of galaxies at each classification is indicated. Error bars are mean errors derived as $\sigma_{1} / \sqrt{n}$, where $\sigma_{1}$ is the standard deviation.

look at multi-feature systems. Stage $\mathrm{SO}^{+}$is a part of the CVRHS sequence where galaxies that are likely highly evolved are mixed with others that have been environmentally modified. By "highly evolved," we mean that well-defined stellar rings and lenses are end-products of long-term internal evolution of what were originally zones of intense star formation. By "environmentally modified," we mean that these same galaxies were stripped of their gas which eventually greatly lowered or even turned off the star formation in their resonant features, allowing secular evolution of the stellar mass distribution to eventually reduce the contrast of the features. Thus, "highly evolved" and "environmentally modified" are not mutually exclusive categories.

\subsection{1. $\mathrm{SO}^{+}$Galaxies: Diversity at a Single Stage}

In optical imaging, late S0s can be dusty, and some galaxies classified in the $B$ band as stages S0/a to Sab (e.g., NGC 1079, $1291,2775,4594)$ can appear to be stage $\mathrm{SO}^{+}$in the mid-IR. Forty-one cases of mid-IR type $\mathrm{S}^{+}$are collected in the montages of Figure 12. Most interesting is that 20 of these objects are of family SA (see Table 6 classification in each frame), and in seven of these, a bright knotty ring is the main morphological feature. If rings are best understood as resonant products of secular evolution in barred galaxies (Buta \& Combes 1996), then these nonbarred cases with bright stellar or star-forming rings are a rather important subset to know about.
Invariant manifold theory (Romero-Gómez et al. 2006, 2007; Athanassoula et al. 2009a, 2009b) also describes the structure and secular evolution of rings, but would also have trouble explaining rings in nonbarred galaxies. The large number of nonbarred $\mathrm{S}^{+}$galaxies in the sample differs from the NIRSOS sample, where $\mathrm{SB}^{+}$and $\mathrm{SAB}_{0}^{+}$galaxies are more abundant (Laurikainen et al. 2013). This could largely be due to the $S^{4} G$ sample bias toward gas-rich S0s.

The following galaxies in Figure 12 deserve special attention because they are good examples of the characteristics they display:

NGC 1553-This galaxy, classified as type $\mathrm{SA}\left(\mathrm{rl}, \mathrm{nr}^{\prime} 1\right) 0^{+}$, is the well-known lens (here a ring-lens) galaxy studied by Kormendy (1984). The lens is significantly elongated in projection, as are the isophotes just outside this zone. In the outer regions, however, the isophotes are nearly circular. The lens appears to be part of an inclined, embedded disk (see also Section 4.4.2). The galaxy also has a nuclear bar that was detected in a near-IR image (Laurikainen et al. 2011).

NGC 4250-This galaxy, classified in Table 6 as type $\left(\mathrm{R}_{1}\right)$ $\mathrm{SAB}(\mathrm{rl}, \mathrm{nl} !) 0^{+}$, is noteworthy for its remarkably high surface brightness nuclear lens. The feature has an angular diameter of $16^{\prime \prime}$ and a linear diameter of about $2.4 \mathrm{kpc}$ for a distance of 29.4 $\mathrm{Mpc}\left(\mathrm{NED}^{27}\right)$. This is larger than the typical nuclear ring, but is

\footnotetext{
${ }^{27}$ NASA/IPAC Extragalactic Database, http://nedwww.ipac.caltech.edu
} 

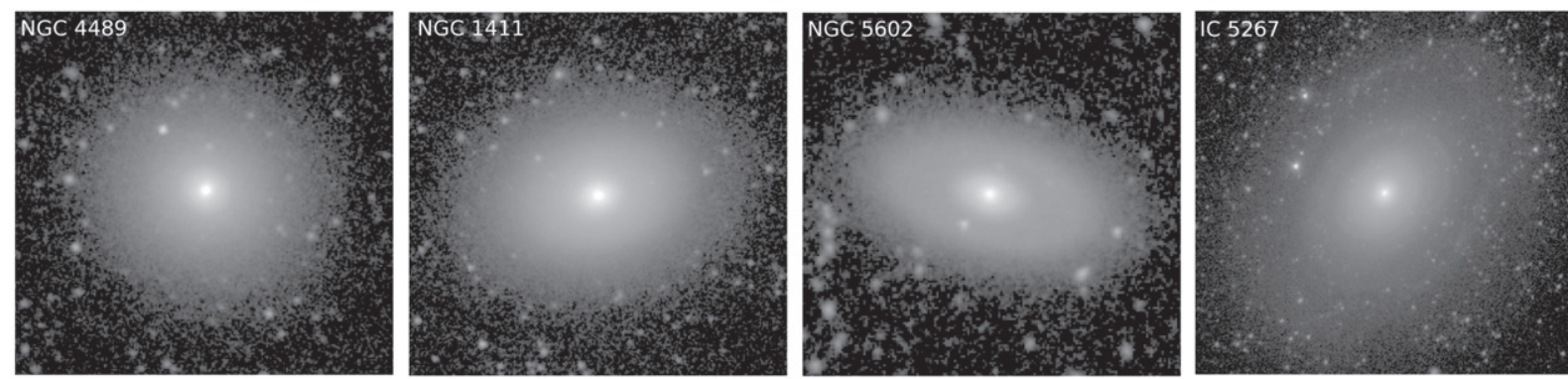

SA
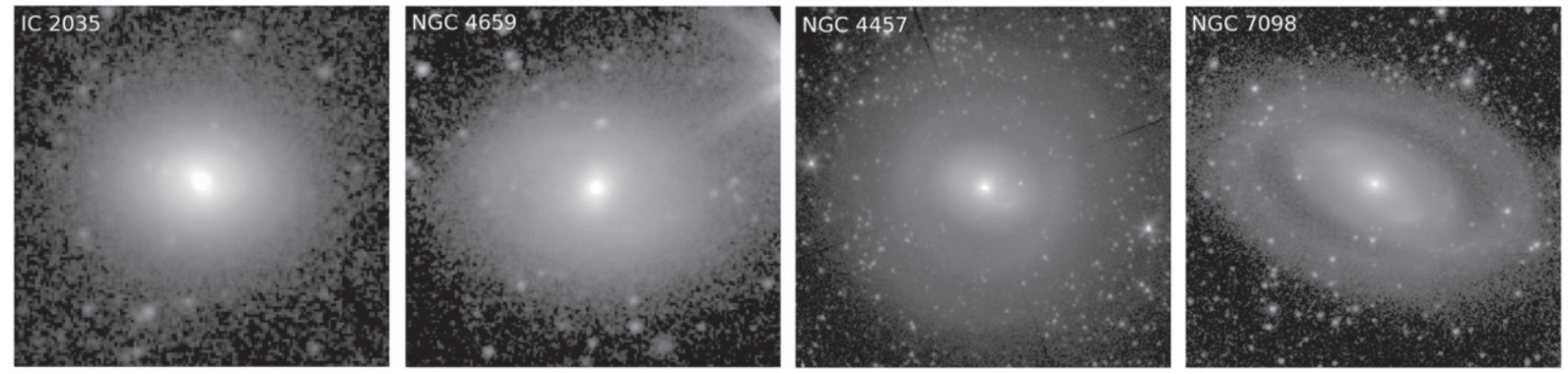

SAB

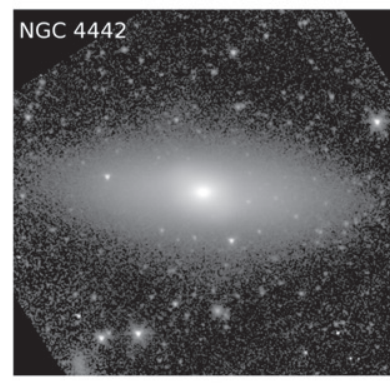

$\mathrm{SO}^{-}$

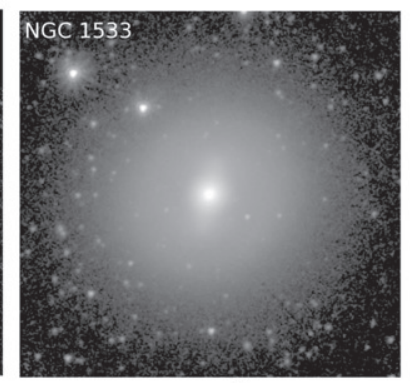

$\mathrm{SO}^{\circ}$

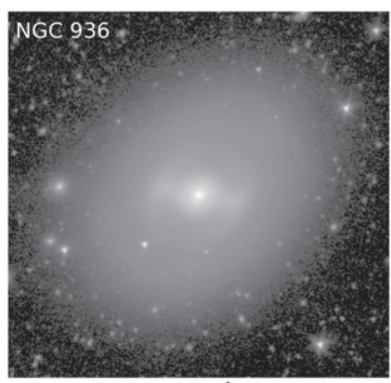

$\mathrm{SO}^{+}$

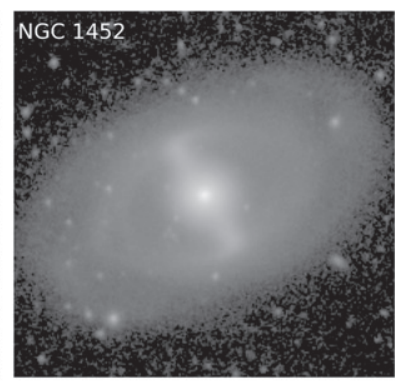

$\mathrm{SO} / \mathrm{a}$

Figure 10. Montage of early-type galaxies covering some of the range in mid-IR morphologies of S0 and S0/a galaxies. Unless otherwise noted, all of the images displayed in this and succeeding figures are in the IRAC $3.6 \mu \mathrm{m}$ filter, and are in units of mag $\operatorname{arcsec}^{-2}$ within the range of surface brightnesses given in the caption for each galaxy in Figure 1. Figure 1 numbers are given in Table 2.
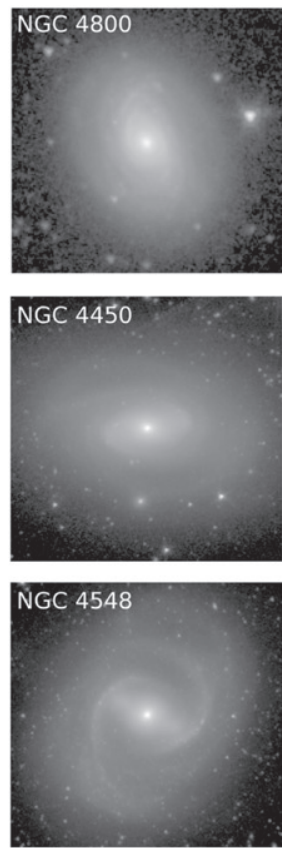

$\mathrm{Sa}$
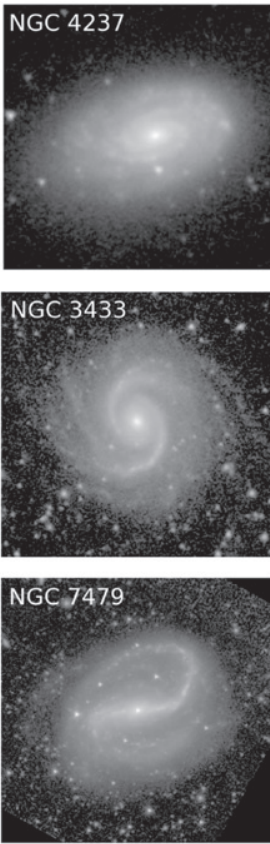

$\mathrm{Sb}$
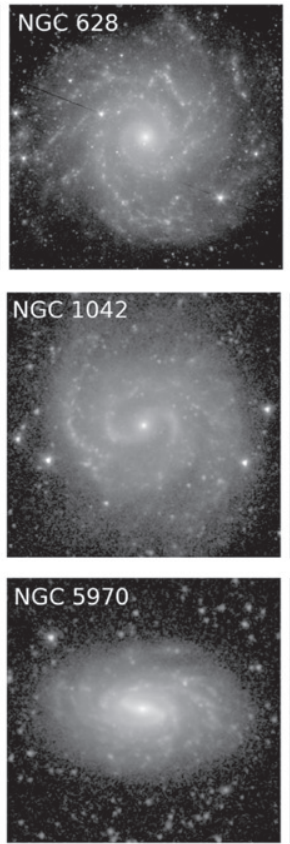

Sc
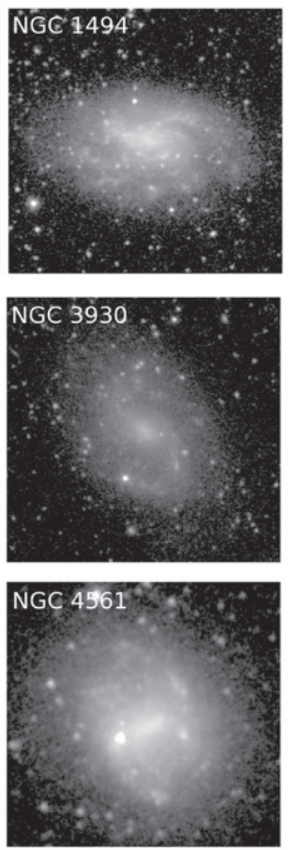

Sd
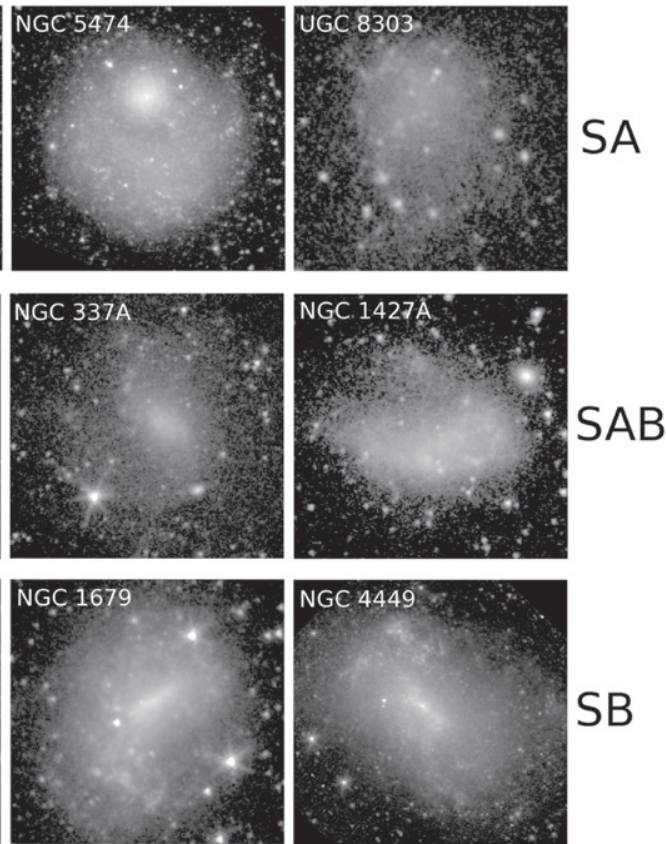

$\mathrm{Sm}$

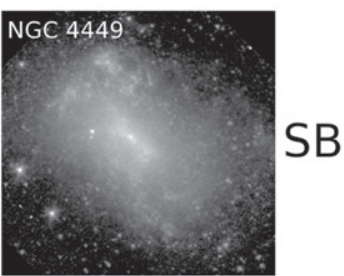

Im

Figure 11. Montage covering some of the range in mid-IR morphologies of spiral and irregular galaxies. 

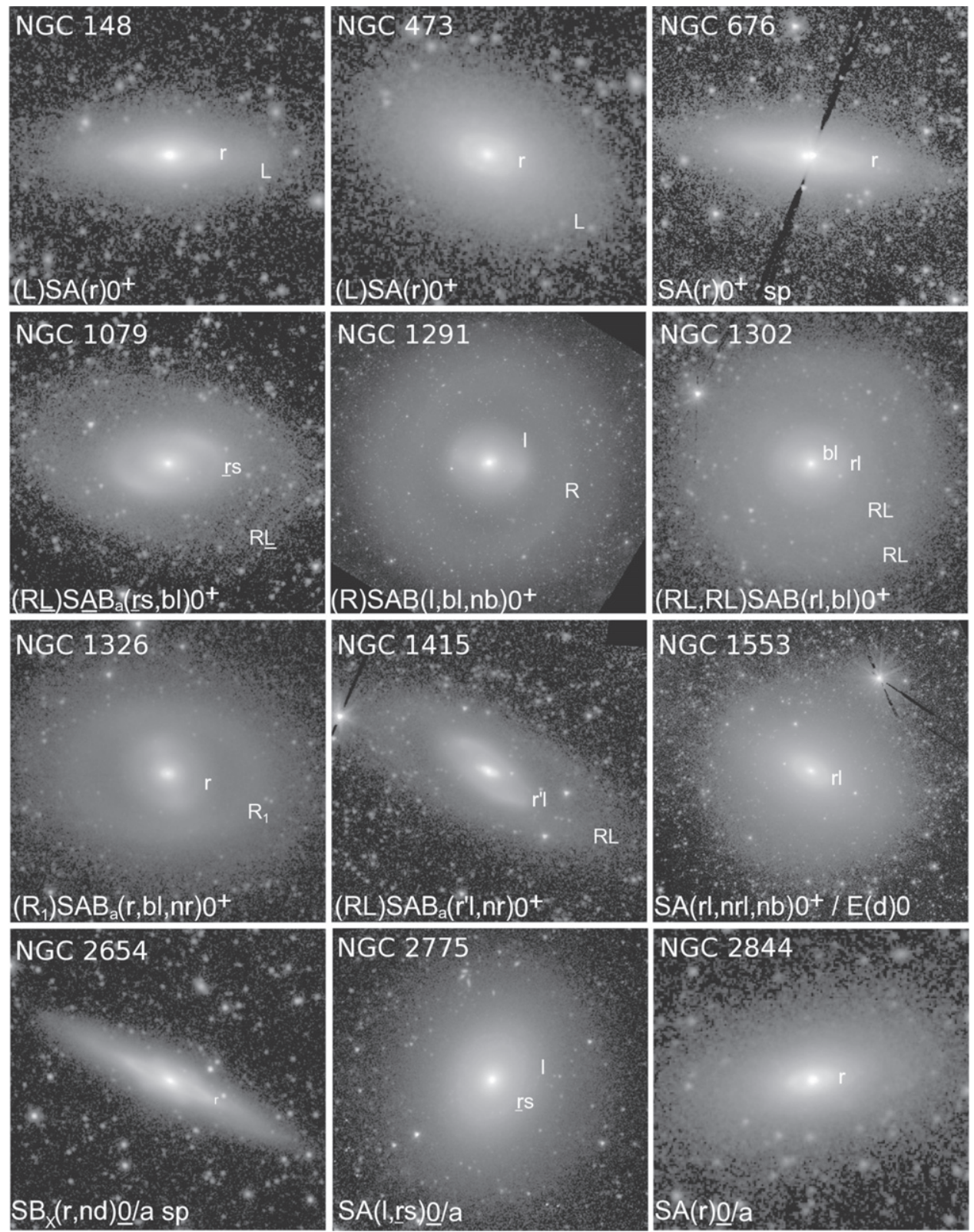

Figure 12. Montages showing the diversity of the mid-IR morphologies of $\mathrm{S}^{+}$galaxies. In addition to the galaxy name, each frame includes the final mean classification from Table 6 . The most important inner and outer variety features are labeled on the images.

within the range of sizes of these features (Comerón et al. 2010). The presence of a very faint, only slightly elongated outer ring indicates that NGC 4250 may be inclined as little as $30^{\circ}$. Thus, the near circular shape of the nuclear lens is intrinsic, while the inner ring-lens is highly elongated, both of which are typical characteristics (Buta 1995, 2012, 2013).

NGC 4344 and NGC 4451-Two low-luminosity members of the Virgo Cluster with small cores and bright partial inner rings of star formation. The small cores suggest that the rings are embedded within Kormendy spheroidals (Kormendy \& Bender 2012). The classification for both galaxies in Table 6 is $\mathrm{SA}(\mathrm{r}) 0^{+}[\mathrm{c}] / \mathrm{Sph}$, where the $[\mathrm{c}]$ indicates a small central concentration for such early-type galaxies. As noted by Ilyina \& Sil'chenko (2011), such star-forming rings (prominent in UV images) are unusual for lenticulars, especially nonbarred lenticulars such as NGC 4344 and 4451. Since bar dynamics are unlikely to account for these rings, an interaction in a cluster environment (e.g., accretion of a small gas-rich companion) may explain them. Ilyina et al. (2014) have also argued that accretion of gas from a large, gas-rich companion can account for similar rings in other $\mathrm{S} 0$ galaxies. Sph galaxies are discussed further in Section 4.5.

$N G C$ 4594-The $3.6 \mu \mathrm{m}$ image reveals a well-defined outer ring and a smooth, bright inner disk. The absence of a clear $\mathrm{X}$ feature suggests the galaxy is nonbarred. Classified as type SA (s)a sp in RC3 and $\mathrm{Sa}^{+} / \mathrm{Sb}^{-}$in the Carnegie Atlas of Galaxies (Sandage \& Bedke 1994), the $S^{4} G$ image reveals little or no clear spiral structure in this well-studied galaxy.

NGC 7742-This galaxy, which is known to exhibit counterrotation (de Zeeuw et al. 2002), has a single bright inner ring and a much fainter, larger ring that is not likely to be an outer ring. The latter could be a weak pseudoring formed by faint spiral arms. The two features are the reason for the Phase 1 variety "(rr)" in Table 2. A double inner variety was also 

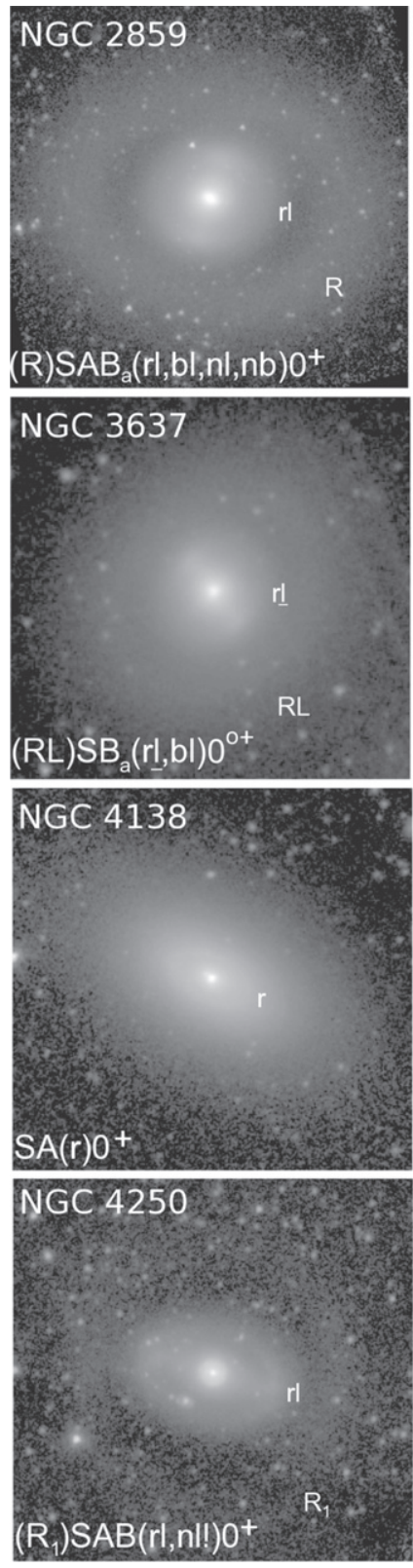
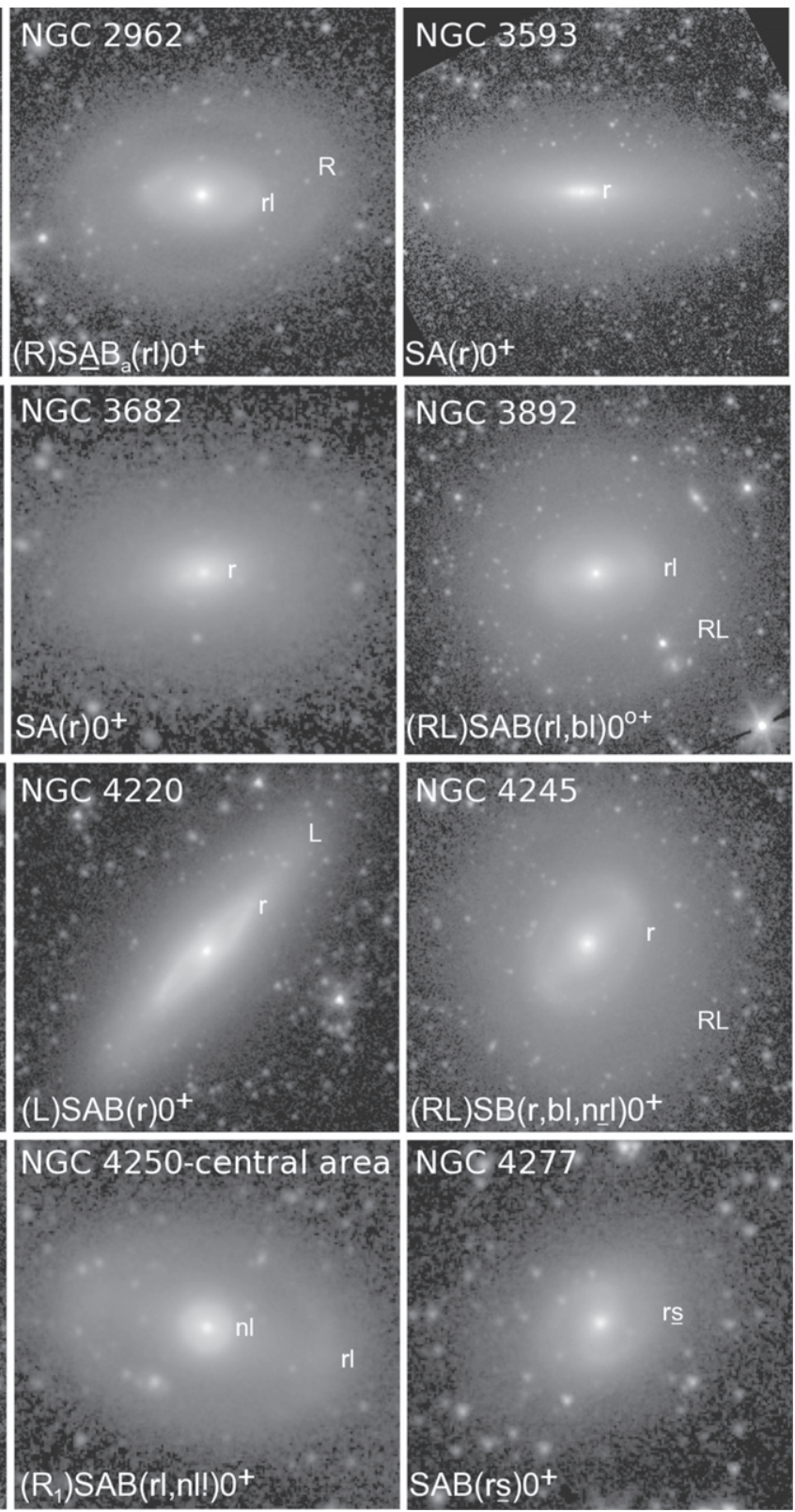

Figure 12. (Continued.)

recognized in a NIRSOS $K_{s}$-band image by Laurikainen et al. (2011).

ESO 358-25-This galaxy is distinctive in the $\mathrm{S}^{4} \mathrm{G}$ sample because it appears to include a small, very bright ring in the central area, but no clear trace of a nucleus or central concentration. The galaxy is included in the ACS Fornax Cluster Survey (Jordán et al. 2007) as object FCC 152, and a high resolution color image posted on the ACSFCS website shows a small blue ring of knots, but again little evidence of a central nucleus. ESO $358-25$ is an intermediate to low luminosity member of the Fornax Cluster similar to other galaxies in the Virgo Cluster. The classification in Table 6 is $\mathrm{SA}(1, \mathrm{r}) 0^{+}[\mathrm{d}] / \mathrm{Sph}$, similar to $\mathrm{NGC} 4344$ and 4451 , but with even less central concentration than those Virgo Cluster galaxies.

Other galaxies in Figure 12 show what we mean by lenses (1), ring-lenses $(\mathrm{rl})$, and pseudoring lenses $\left(\mathrm{r}^{\prime} 1\right)$, or the outer counterparts of these features. For example, NGC 1291 shows a very bright inner lens at about half the diameter of a subtle outer ring embedded in a more circular background. The feature has a shallow brightness gradient interior to a sharp edge, but no rim enhancement. The galaxy is virtually face-on, so the slightly elongated shape of the (1) is intrinsic.

NGC 4457 shows a similar slightly elongated inner lens in a face-on disk. But most interesting are the conspicuous inner lenses in the nonbarred galaxies NGC 5602 and IC 2764. Both of these also have a prominent outer ring-lens (RL).

NGC 2859 is also very similar to NGC 1291 except the rim of the prominent (1) is slightly enhanced, and the classification is $(\mathrm{rl})$. Other classified inner ring-lenses in Figure 12 include NGC 2962, 3637, 3892, 4250, and 5770. The classified inner rings (r) in NGC 1326 and 4245 tend to have more of an enhancement, but even these features are not especially strong as inner rings. NGC 1415 is classified as having an $\left(\mathrm{r}^{\prime} 1\right)$ (where 

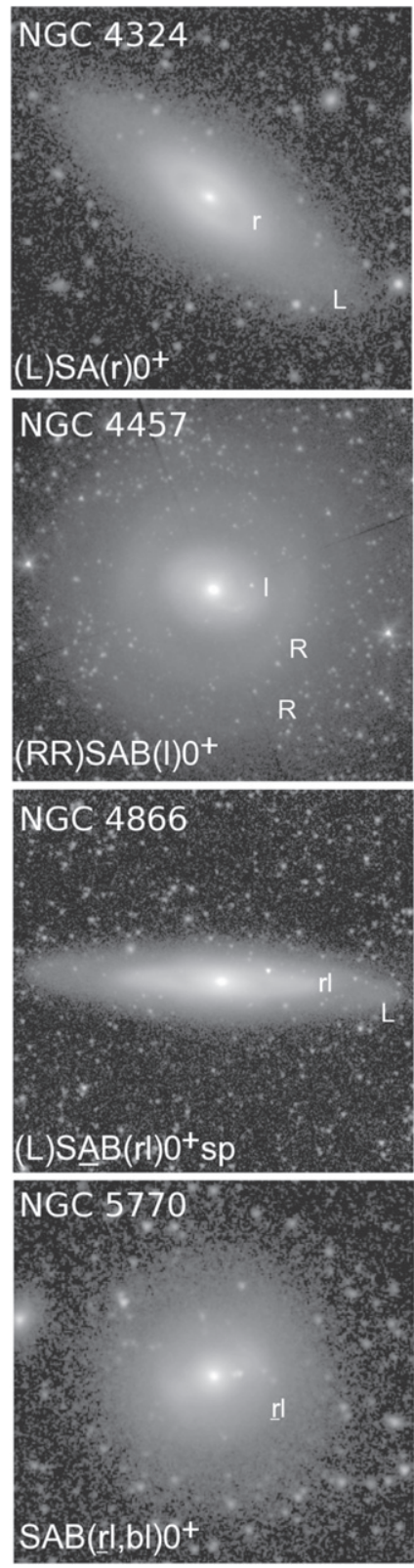
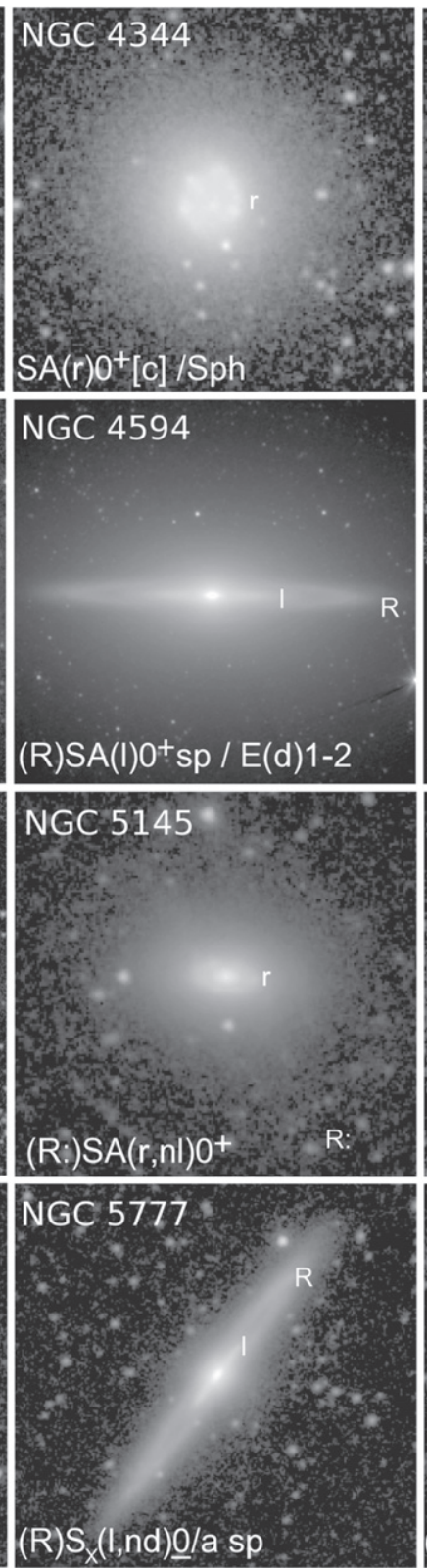

Figure 12. (Continued.)
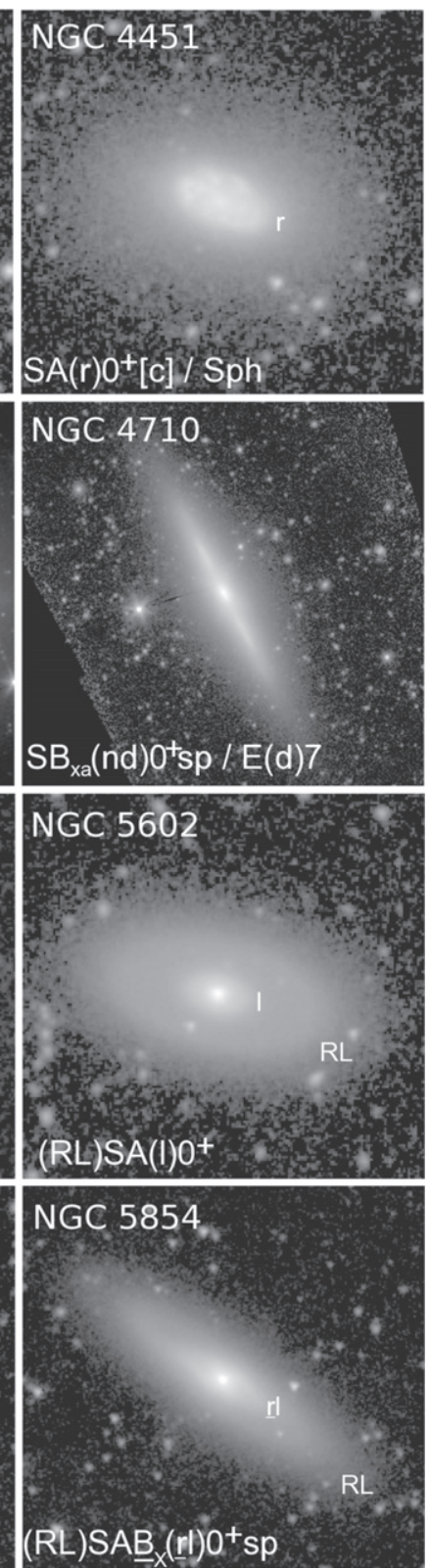

" $\mathrm{r}$ ", is an alternate notation for inner pseudoring) because of a subtle spiral structure at the rim of the lens.

\subsubsection{Double Inner and Outer Varieties}

Most of the galaxies in Figure 12 are single inner and outer variety systems, i.e., one outer feature $\left(\mathrm{R}, \mathrm{R}^{\prime}, \mathrm{RL}, \mathrm{L}\right)$ and/or one inner feature $\left(\mathrm{r}, \mathrm{rs}, \mathrm{r}^{\prime} \mathrm{l}, \mathrm{rl}, \mathrm{l}\right)$ between CVRHS classification bracketts. Several multiple inner and outer variety systems are shown in Figures 13 and 14. The recognized features are labeled with the classifications shown.

Comments on the six galaxies in Figure 13 are as follows: NGC 289-An extensive, smooth spiral showing multi-scale individual, pseudoring-like patterns. The inner (rs) envelops a weak bar, while the second (rs) is morphologically distinct and about the twice the diameter of the first (rs). In the outer regions, an outer pseudoring-lens, $\mathrm{R}^{\prime} \mathrm{L}$, is seen as a subtle enhancement. Not seen in the illustration is a fourth, much fainter feature (an $\mathrm{R}^{\prime}$ ) approximately twice the diameter of the $\mathrm{R}^{\prime} \mathrm{L}$.

NGC 986-A barred spiral with a strong s-shaped pattern mixed with a clear inner pseudoring pattern. Both the (s) and the (rs) are nevertheless normal-looking features. The main arms of NGC 986 also form an outer pseudoring.

NGC 4698-The Table 6 classification recognizes two inner rings and a single, much larger outer ring that is outside the illustrated frame. The two inner rings are closely spaced and very subtle, and could be parts of a tightly wound spiral pattern. The inner rings are embedded in a much rounder component.

NGC 5055-The two inner features consist of a well-defined (rl) and an (rs) about twice as large. The latter is fairly open but is still a distinct pattern.

NGC 5364-This galaxy has an extremely well-defined spiral pattern outside of a bright inner ring. What is interesting 

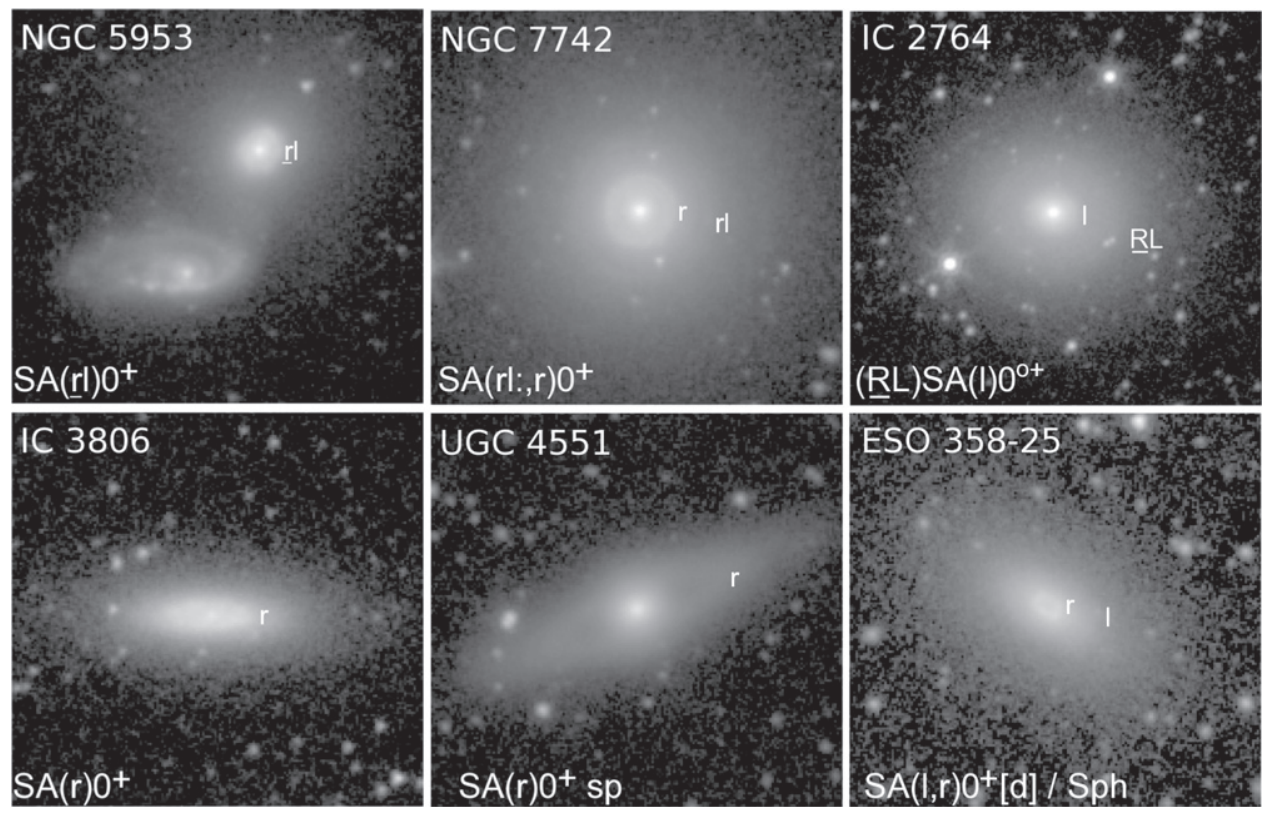

Figure 12. (Continued.)
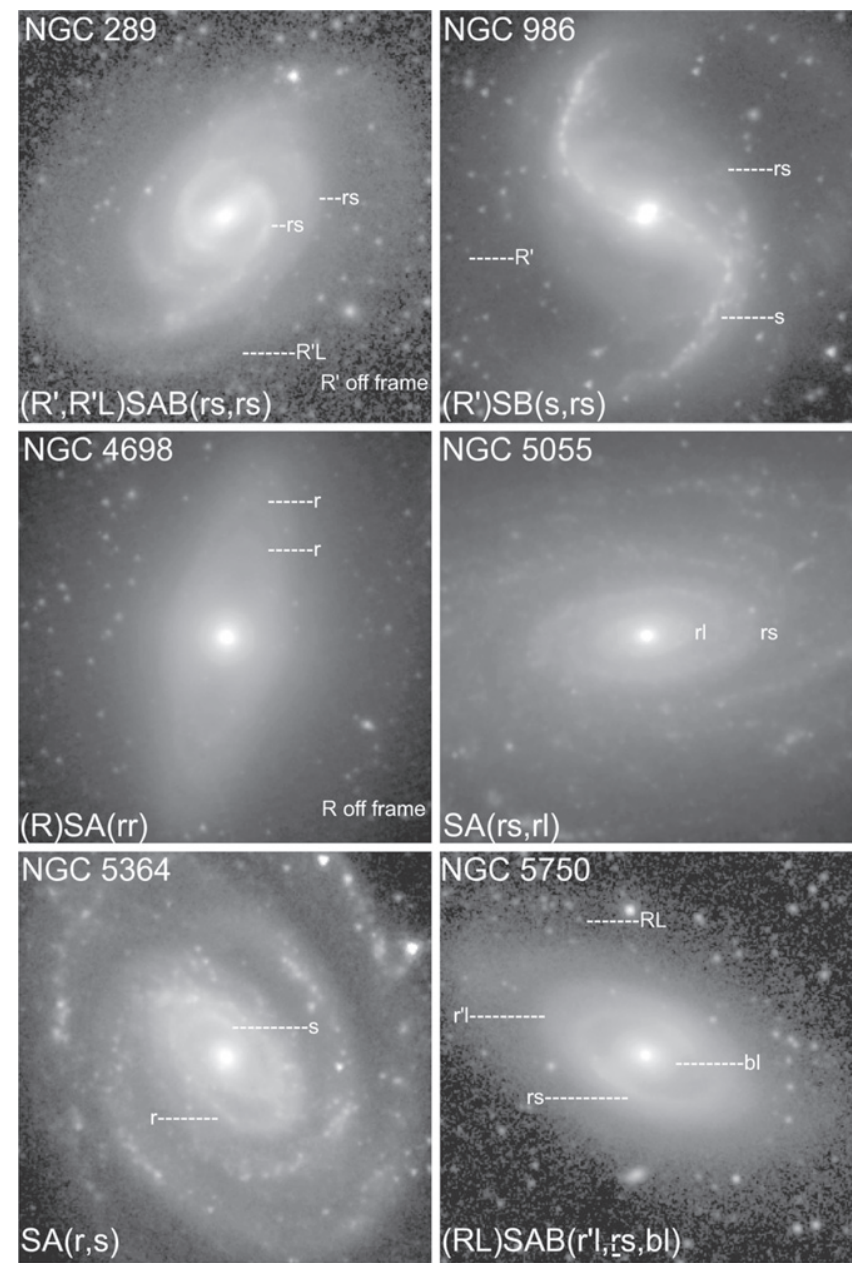

NGC 5055

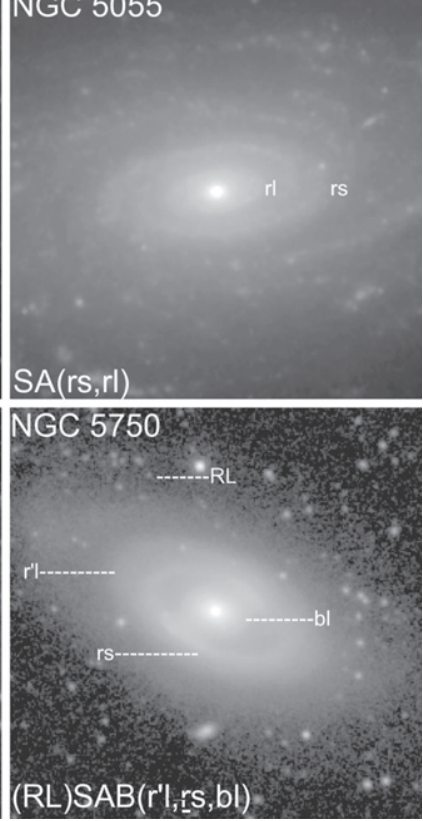

Figure 13. Six galaxies with two recognized inner features in the CVRHS classifications in Table 6. These features are labeled on each frame to show what the symbols are referring to. In two cases (NGC289 and 4698), an additional outer feature is outside the field of the image.

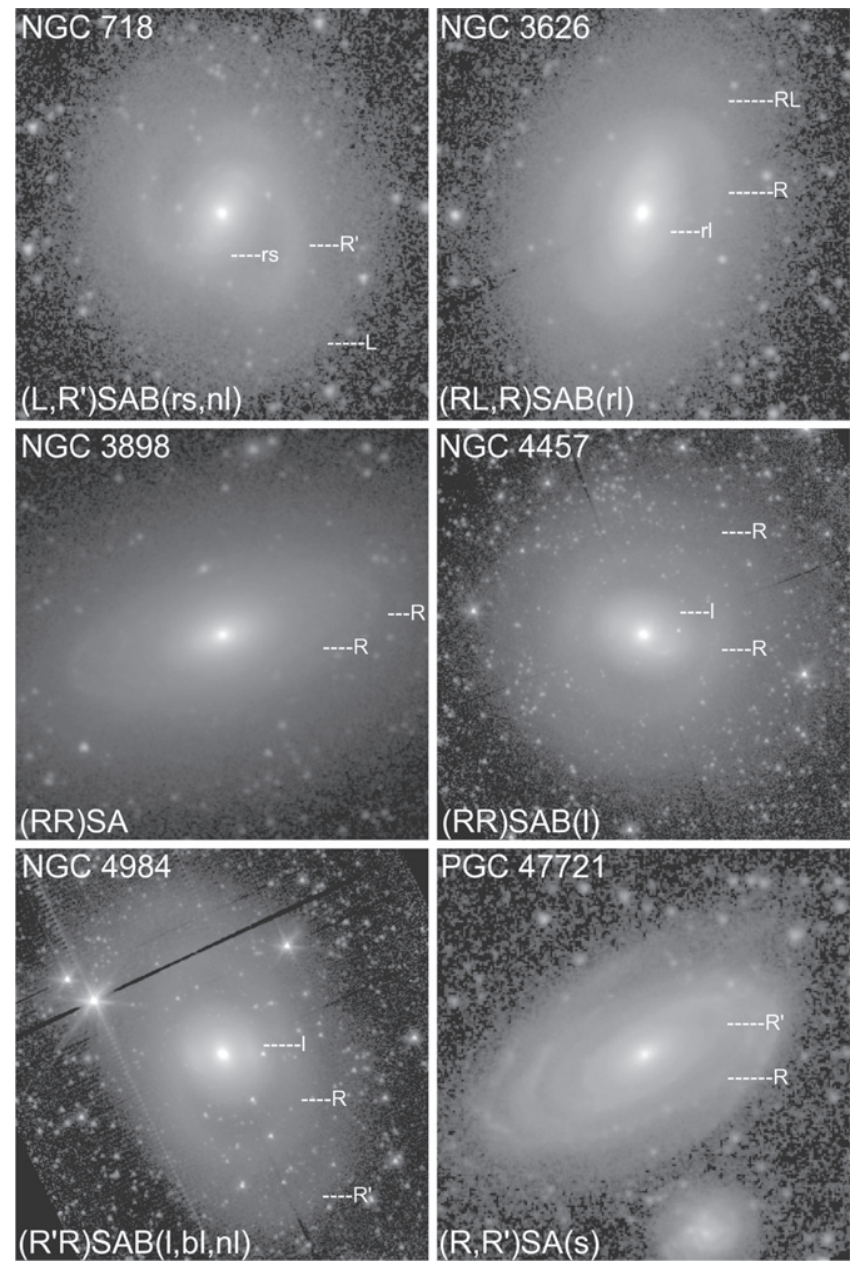

Figure 14. Six galaxies with two recognized outer features in the CVRHS classifications in Table 6. These features are labeled on each frame to show what the symbols are referring to. In some cases, an inner feature is also labeled. 
is the unrelated spiral pattern lying inside the inner ring. The two patterns together form the unusual variety $(\mathrm{r}, \mathrm{s}$ ) (as opposed to (s,rs) for NGC 986).

NGC 5750 - This early-type system shows a mostly closed inner ring enveloping a foreshortened bar. Close outside this ring is a second feature we classify as an $\left(\mathrm{r}^{\prime} 1\right)$, giving the galaxy a double variety character. A partial outer ring-lens is seen beyond these two features.

Comments on the six galaxies in Figure 14 are as follows: NGC 718-This galaxy has a well-defined outer pseudoring, bar, and inner pseudoring, but this whole pattern set is embedded in a relatively uniform background which we recognize as an outer lens $(\mathrm{L})$ that extends to more than twice the diameter of the $\mathrm{R}^{\prime}$.

NGC 3626-The (rl) and ( R) are normal features, but like NGC 718, these are embedded in a more extensive background including what we classify as an (RL).

NGC 3898 - an early-type nonbarred galaxy having two closely space large rings that we interpret as a pair of outer rings, (RR).

NGC 4457-The deep $3.6 \mu \mathrm{m}$ image reveals not only the previously known bright outer ring in this well-known Virgo Cluster galaxy (Sandage 1961), but also what appears to be a second outer ring at roughly twice the radius of the first feature. Both features are very nearly circular. The second ring is also detectable in an SDSS color image. An unsharp-masked nearIR image previously revealed the weak bar in the galaxy (Laurikainen et al. 2011).

NGC 4984-The inner regions consist of a normal lens and faint bar with ansae. Surrounding this zone is an outer ring, but most unusual is the second outer feature, an $\mathrm{R}^{\prime}$, at approximately twice the diameter of the first outer ring. This accounts for the outer variety $\left(\mathrm{R}^{\prime} \mathrm{R}\right)$.

$P G C$ 47721-An M81-like galaxy which has what appears to be a mostly closed ring within its main outer spiral pattern. Within this feature is a very large pseudoring. The two features account for the classification of $\left(\mathrm{R}, \mathrm{R}^{\prime}\right)$, although in the absence of a bar, these could also be interpreted as very large inner rings.

\subsection{The Mid-IR Morphology of Bars}

Bars are a logical focus for mid-IR morphological examination. It is well-known that some bars which appear weak or even absent in blue light imaging can be more prominent in IR bands (e.g., Eskridge et al. 2000). Heavy internal extinction (due, for example, to strong leading dust lanes or other factors) can account for some of these, while stellar populations can account for others in the sense that IR bands are more sensitive to the older stellar populations seen in many bars than is the $B$ band. In paper I, we argued that the "stronger bar effect" is apparent in $\mathrm{S}^{4} \mathrm{G}$ images, but that the rankings of bars (by actual strength) are not greatly changed. What we mean by this is that, while weaker bars can appear more conspicuous in the IR as compared to blue light, such that a $B$-band SAB-type bar can be classified as IR type $\mathrm{SB}$, a $B$-band SB-type bar has no stronger category to be placed in, yet it also looks stronger in the IR. The rankings of bars (strong versus weak) thus is not changed greatly from blue light to the mid-IR. This does not undermine the case for IR imaging: it is still best to judge the importance of any bar in an IR waveband.

Bars can also be reliably recognized in both face-on and edge-on galaxies, as well as across the entire CVRHS sequence from stage $\mathrm{S}^{-}$to $\mathrm{Im}$. No other aspect of galaxy morphology has this characteristic, which is considerably enhanced in the mid-IR. In this section, we look at the mid-IR morphology of bars with two goals: (1) to highlight the diversity of bar morphologies, especially among early-type galaxies, and (2) to clarify the meaning of family classifications between early- and late-type galaxies that impacts how we interpret the barred family fraction.

\subsubsection{Ansae Bars and Barlenses}

One of the interesting aspects of the galaxies in Figure 12 is how weak-looking most of the apparent bars are, even if previously classified as SB. For example, NGC 1291 is classified as (R)SB(s)0/a in RC3 and as SBa by Sandage \& Tammann (1981), and yet, viewed against the bright inner lens in a logarithmic, background-subtracted image, the bar classification that seems appropriate for NGC 1291 is SAB at best (see also the dVA). The same is true for NGC 2859 and others shown in the Figure. Only NGC 3637 and 4245 have bars classified as SB in Table 6.

Many of the apparent bars in the galaxies in Figure 12 are of the ansae ("handles") type, where the ends of the bar are defined by subtle brightness enhancements. Martinez-Valpuesta et al. (2007) made a statistical study of these features, and concluded that ansae are mainly a phenomenon of earlytype galaxies and are very rare at stages later than $\mathrm{Sb}$. Figure 15 shows a selection of 12 of the best-defined ansae bars in the $\mathrm{S}^{4} \mathrm{G}$ sample. Because these features are subtle, Figure 16 shows unsharp-masked images of the same 12 galaxies. These show how ansae come in different morphologies, ranging from curved or nearly linear arcs to rounder spots. Although no detailed quantitative measurements have yet been made, SDSS color images and dVA color index maps have indicated that ansae may be purely stellar in nature or include star formation. In Tables 2 and 6, ansae are recognized using the symbols $\mathrm{SB}_{a}$ and $\mathrm{SAB}_{a}$.

Although the detection of ansae does not necessarily require observing galaxies in the mid-IR, mid-IR imaging can still shed some light on the properties of the features, such as their vertical structure. NGC 4216 is the most highly inclined example shown in Figure 15, and in the optical shows strong planar dust. In the mid-IR, the galaxy shows an inner boxy structure and two intense brightness enhancements that, in Figure 16, appear at the ends of the major axis of a thin inner ring. These enhancements are likely to be ansae in the inner ring. Although we cannot rule out that these features are merely due to line-of-sight effects through the major axis points of an inner ring, other nearly edge-on galaxies with highly inclined rings (e.g., NGC 4594 in Figure 12) do not show conspicuous ansae.

The boxy inner zone of NGC 4216 shows a very faint Xpattern in the unsharp-masked image, indicating that the galaxy does indeed have a bar with vertical resonant structure. However, the ansae are clearly much flatter features. Thus, mid-IR imaging shows that a bar in a $B$-band intermediate-type barred spiral consists of a 3D inner section and much flatter ends, consistent with the general structure of bars noted by Athanassoula (2005).

In Figure 15, NGC 7424, a face-on SBcd galaxy, is much later than any ansae barred galaxies identified by MartinezValpuesta et al. (2007). Because bars in extreme late-type spirals can be linear chains of star forming regions, it is not 

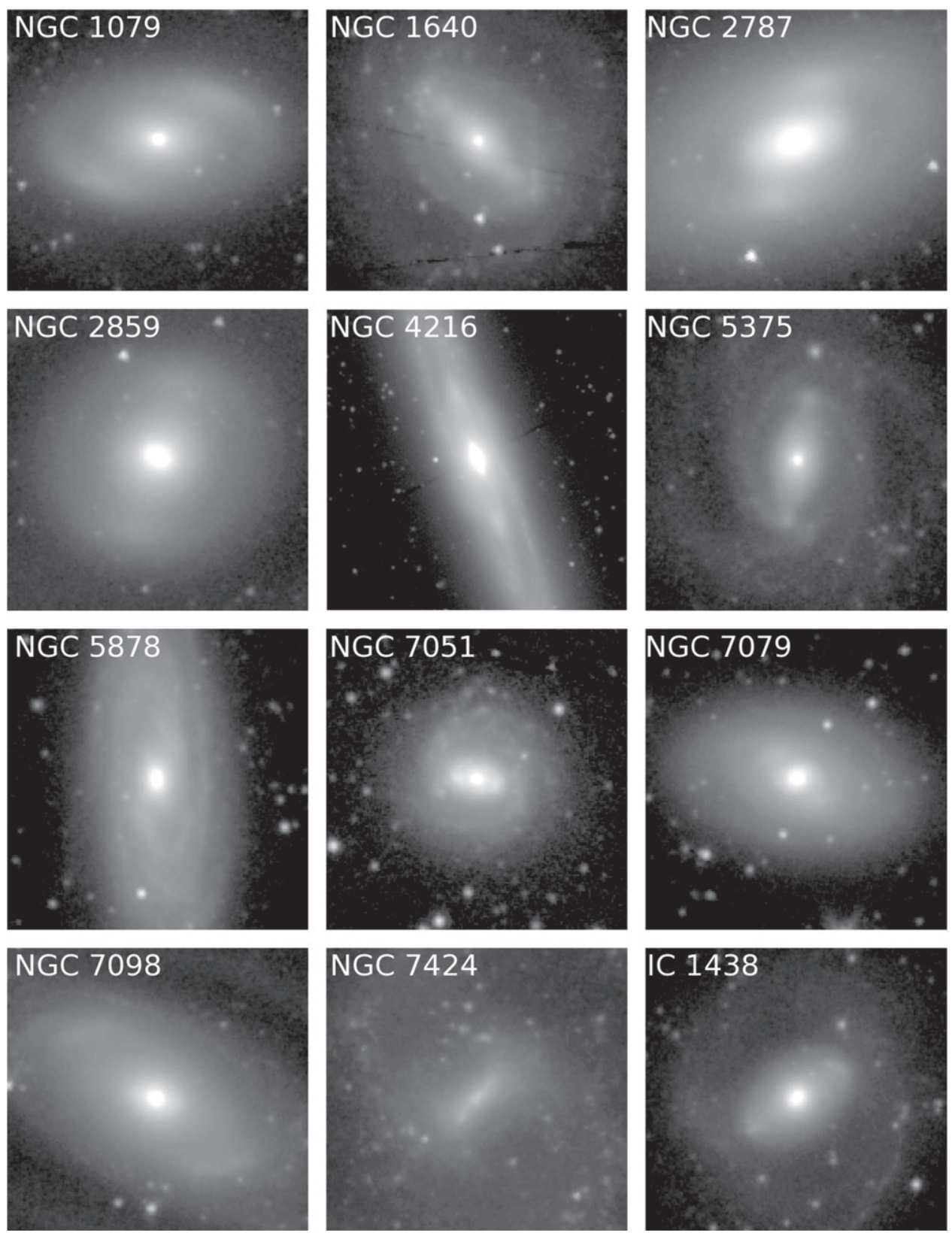

Figure 15. Montage showing the mid-IR morphology of ansae bars in $12 \mathrm{~S}^{4} \mathrm{G}$ galaxies.

clear that the apparent ansae in NGC 7424 are in any way dynamically related to those seen in the other galaxies in Figure 15. Late-type galaxy bars as compared to early-type ones are more carefully examined in Section 4.3.3.

Figure 15 shows an additional feature of barred galaxies only recently recognized. This is the "barlens," symbolized by (bl). A barlens refers to the inner part of a typical early-type galaxy bar. As a class of structures, barlenses were first recognized by Laurikainen et al. (2010), who used high-quality near-IR images and multi-component decompositions to establish the distinct nature of the features. Barlenses can be mistaken for a bulge because they generally have less elongated isophotes than the bar ends. They appear to be part of the bar.

Laurikainen et al. (2013) proposed that a barlens represents an evolution of the bar, and that inner lenses in nonbarred galaxies could be the former inner part of an evolved, disintegrated bar (see their Figure 11). The curved ansae in
NGC 1079 appear to be the bar ends dispersing into the inner ring-lens area. Athanassoula et al. (2014) and Laurikainen et al. (2014) use both numerical simulations and observations to show that barlenses are indeed likely to be the more face-on view of the $3 \mathrm{D}$ inner part of a bar, that when seen edge-on shows the familiar X or box/peanut shape. It is likely that NGC 4216, for example, in Figure 15 would show a barlens in the face-on view, with the barlens corresponding to the broad inner zone.

Five galaxies (NGC 1079, 2787, 2859, 5375, 7079) with a barlens are included in Figure 15, the best example being seen in NGC 2787. More than 70 examples are included in the whole catalog. A barlens and a regular inner lens differ in scale: in a barred galaxy with an (1), the bar typically fills the (1) in one dimension (Kormendy 1979), while the (bl) is smaller than the bar, as measured from the NIRSOS atlas (Laurikainen et al. 2011) and illustrated in Athanassoula et al. (2014). This 

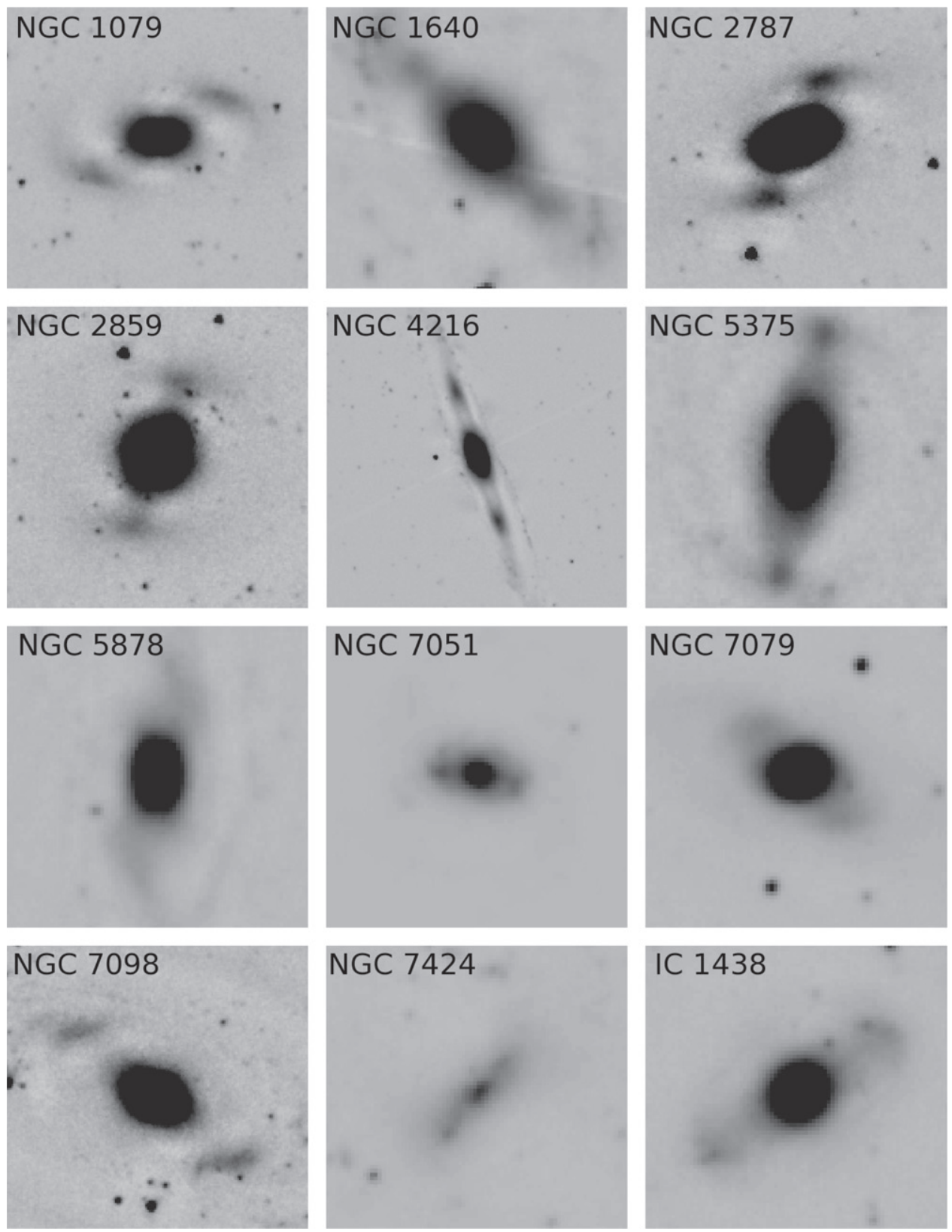

Figure 16. Same galaxies as in Figure 15, displayed using unsharp-masking (and on different scales in some cases) to make the ansae more easily visible. The $3.6 \mu \mathrm{m}$ images are in intensity units.

is in agreement with the theoretical predictions by Athanassoula (2005) about the relative extents of the thick and the thin part of the bar. A (bl) will also generally be larger than and distinct from a nuclear lens (nl). ${ }^{28}$ Laurikainen et al. (2013) showed that ansae occur even in $52 \%$ of galaxies having barlenses, compared to $24 \%$ when no barlens is present. However, multiple lenses are rare in such galaxies.

In spite of what ansae may imply about bar structure and evolution, the features are still poorly understood. No simulations have yet accounted for the diversity of the morphologies of ansae (round spots, arcs, linear shapes), or

\footnotetext{
${ }^{28}$ This is well-illustrated by Buta et al. (2001), who used Fourier analysis to remove the bar of NGC 1433 from a deprojected near-IR $H$-band image, leaving behind "a large round area surrounding the secondary lens and bar," a preliminary isolation of a barlens (see their Figure 5).
}

for their spread in color (young versus old stellar population ansae; e.g., Martinez-Valpuesta et al. 2007; Buta 2013).

\subsection{2. $X$-structures}

$X$-structures are strongly evident in some $\mathrm{S}^{4} \mathrm{G}$ galaxies, five of which are shown in Figure 17. Like the ansae in Figure 16, these unusual patterns can be made more obvious using unsharp-masking (upper right inserts in Figure 17). Detailed observations and numerical simulations (e.g., Athanassoula \& Bureau 1999; Bureau \& Freeman 1999; Bureau et al. 2004; Bureau et al. 2006) have definitively shown that X-structures are related to the edge-on view of a bar. The $\mathrm{X}$ is believed to show the vertical resonant structure of the bar. Often, the $\mathrm{X}$ pattern is a subtle aspect of what is commonly referred to as a "box/peanut bulge" (Luetticke et al. 2000a, 2000b). Here we 


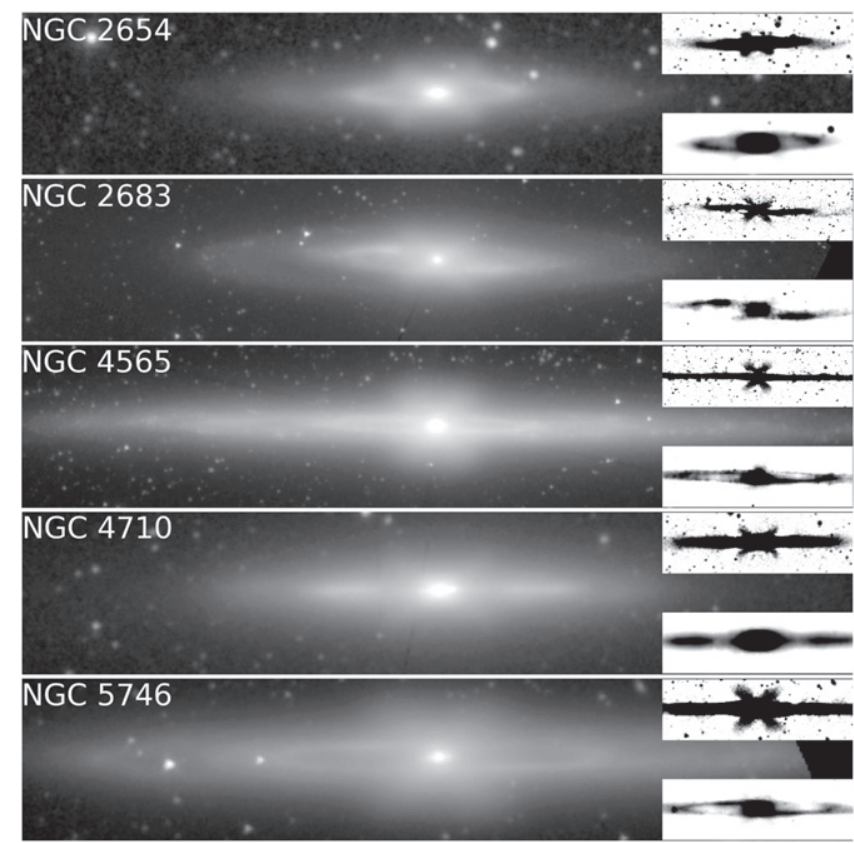

Figure 17. Montage showing the mid-IR morphology of five $S^{4} G$ galaxies showing strong inner X-patterns. The units of these $3.6 \mu \mathrm{m}$ images are mag $\operatorname{arcsec}^{-2}$ over the same ranges for the galaxies given in the captions to Figure 1. The upper right inserts show unsharped-masked versions of the intensity images after subtraction of a heavily median smoothed image. These make the $\mathrm{X}$-patterns more visible. The lower right inserts show the same unsharp-masked images of the inner disk regions to highlight inner rings and bar ansae. The scales of these inserts are twice that of the upper right inserts, except for NGC 5746 where the scale of the lower right insert is 1.25 times that of the upper right insert.

follow the de Vaucouleurs nomenclature and use the generic term X-structure for any box/peanut $/ \mathrm{X}(\mathrm{B} / \mathrm{P} / \mathrm{X})$ structure or bulge. In Table 6 , these are recognized using the symbols $\mathrm{SB}_{\mathrm{x}}$ and $\mathrm{SAB}_{\mathrm{x}}$ (Table 1). For edge-on galaxies, the $\mathrm{SB}$ in $\mathrm{SB}_{\mathrm{x}}$ is inferred if the $\mathrm{X}$ is especially strong, but if the $\mathrm{X}$ is fairly weak, then $\mathrm{SAB}_{\mathrm{x}}$ is used instead.

For all of the galaxies in Figure 17, internal dust complicates the view of the $\mathrm{X}$ in blue light but did not prevent the box/ peanut shape from being detected. All were recognized as peanut or boxy bulges by Luetticke et al. (2000a), based on inspection of Digitized Sky Survey images. Most interesting is how in four of these galaxies, the X-pattern is situated within a conspicuous inner ring or pseudoring whose projected shape indicates that the galaxies are not exactly edge-on. These rings were not necessarily obvious in blue light. The lower right inserts in Figure 17 show unsharp-masked images of the rings that reveal the presence of ansae in at least two cases (NGC 2654 and 2683). NGC 4710 appears exactly edge-on and only ansae are visible on each side of the center. Because inner rings and pseudorings commonly envelop bars in more face-on galaxies, the detection of strong $\mathrm{X}$ patterns situated within bright inner rings helps to clinch the argument that X-patterns are related to bars. The detected inner ring in NGC 4565 is also described by Kormendy (2012) and de Looze et al. (2012).

The five galaxies in Figure 17 also show that the width of the $\mathrm{X}$ pattern relative to its vertical height can vary. In NGC 4565 , for example, the nearly round pseudobulge shows a tighter $\mathrm{X}$ feature than is seen in NGC 2654, 2683, and 5746, suggesting that our perspective on the bar of NGC 4565 must be more endon than for these galaxies.
Table 6 includes more than 60 visually recognized box/ peanut/X-galaxies in the $S^{4} G$ sample. While $X$-patterns are often most easily recognized in nearly edge-on galaxies, they are also seen in much less inclined galaxies (e.g., the dVA; Erwin \& Debattista 2013). An example in the $S^{4} G$ sample is NGC 5377 (paper I). In such cases, the classifications $\mathrm{SB}_{\mathrm{x}}$ and $\mathrm{SAB}_{\mathrm{x}}$ are not inferences of a bar as they would be for edge-on galaxies.

Note that Erwin \& Debattista (2013) did not show or statistically examine X-shaped bars, only box/peanut features, or bars having spurs. They examined a few moderately inclined galaxies with X-shapes from the NIRS0S atlas assuming that physically they represent the same phenomenon. The inclination distribution of the galaxies with $\mathrm{X}$-shaped bars for the combined S4G+NIRS0S sample is given in Laurikainen et al. (2014 see their Figure 2). This study showed that X-shapes appear only in bright galaxies, and not in the latest Hubble types. The identifications of the X-shapes in Laurikainen et al. (2014) was based on unsharp masks made for the complete $\mathrm{S}^{4} \mathrm{G}$ and NIRSOS samples.

\subsubsection{Early-Versus Late-type Bars}

Elmegreen \& Elmegreen (1985) examined the photometric properties of bars over a range of types, and found a dichotomy: early-type galaxy bars are long, strong, and have a relatively flat luminosity profile, while late-type bars are short, relatively weak, and have an exponential luminosity profile. This dichotomy is evident also in $S^{4} \mathrm{G}$ images. By "strong bar," we will generally mean a bar having a maximum relative bar torque parameter $Q_{b} \geqslant 0.2$ (Buta et al. 2006).

CVRHS family classifications provide visual information on apparent bar strength in galaxies, but little insight on the actual diversity of bar morphologies. The recognition of ansae bars, $\mathrm{X}$-structures, and barlenses (through $\mathrm{SB}_{a}, \mathrm{SB}_{\mathrm{x}}$, etc, and (bl), respectively) provides extra information about a bar in a given galaxy, but fails to account for the significant differences between the bars seen in early- and late-type galaxies. In Section 3.5 it was shown that the distribution of family classifications in the $S^{4} G$ catalog depends on mid-IR stage, with SA classifications being most abundant in the type range S0/a to Sc, and SB classifications being most abundant in the Scd-Sm type range. The barred family classification fractions were found to be $55 \%$ in the range $\mathrm{S} 0 / \mathrm{a}-\mathrm{Sc}$ and $81 \%$ in the range $\mathrm{Scd}$ to $\mathrm{Sm}$.

At stage Scd, bars begin to show a distinct knotty morphology. Ansae, barlenses, and X-patterns seem to be no longer relevant. To see this, Figure 18 shows the bar regions of six relatively low-inclination $\mathrm{S}^{4} \mathrm{G}$ galaxies having mid-IR stages in the range $\mathrm{Sa}$ to Sc, while Figure 19 shows the bar regions of six galaxies in the type range Scd-Sdm. In each case, the IRAC $3.6 \mu \mathrm{m}$ image has been deprojected and rotated to make the bar horizontal. Foreground and background objects have also been removed.

In the Figure 18 montage, NGC 4314 has a prominent barlens, and indeed the feature was used as a prototypical example of a (bl) in the NIRSOS atlas (Laurikainen et al. 2011). Laurikainen et al. (2014) also show further evidence in favor of the barlens interpretation for this galaxy (see their Figure 1). NGC 5375 has two strong circular ansae in addition to a barlens (see also Figures 15 and 16), NGC 1300 and 1365 have very strong spirals breaking from the ends of their bars, while NGC 1042 and 5970 each have a bar with a 


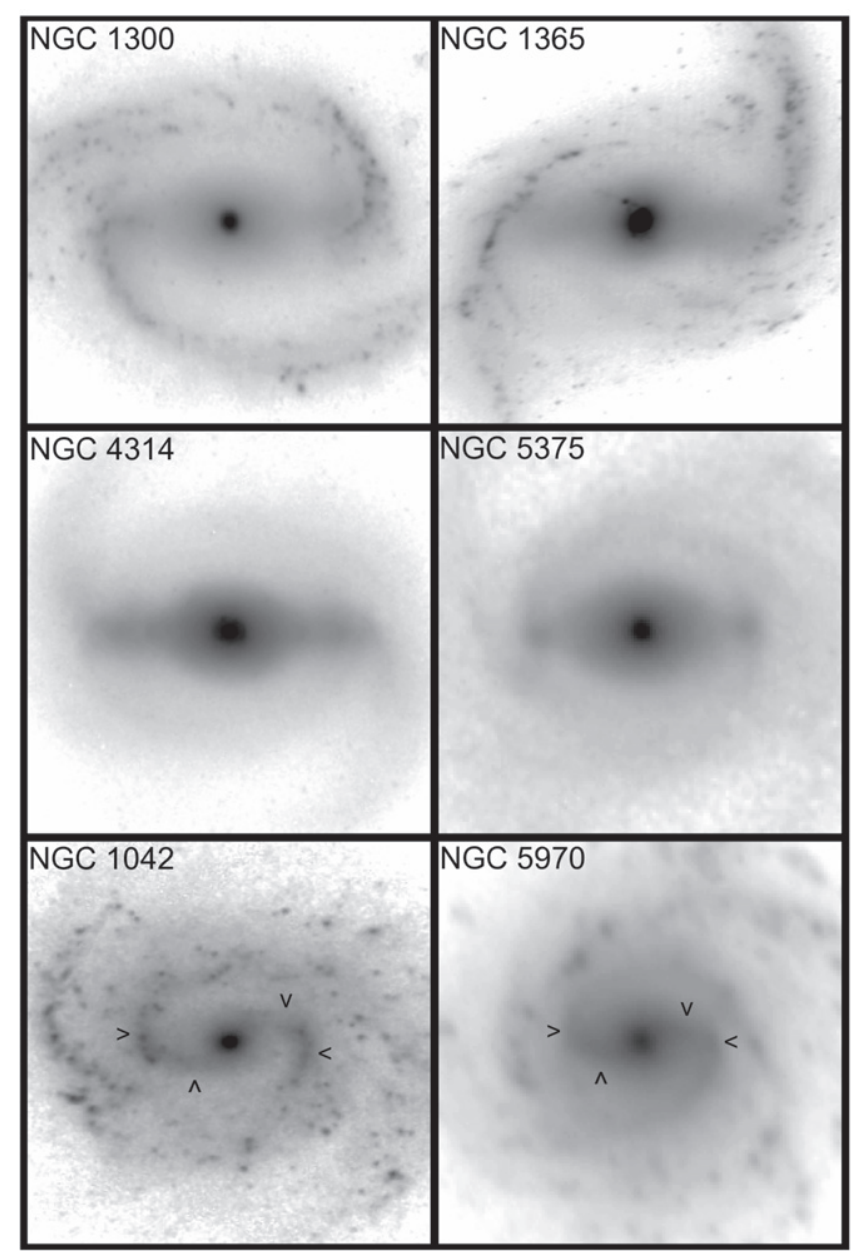

Figure 18. Deprojected $3.6 \mu \mathrm{m}$ images of 6 galaxies in the mid-IR type range $\mathrm{S} 0 / \mathrm{a}$ to Sc, rotated such that the bar is oriented horizontally. The bars in the lower two examples (NGC 1042 and 5970) show a spiral character that is outlined with the circumflexes. Units of the images are mag $\operatorname{arcsec}^{-2}$.

clear spiral character (highlighted by the circumflexes) that, at least in the case of NGC 1042, is not likely to be due to a projection effect between the 3D inner part of the bar and the flatter bar ends (e.g., Erwin \& Debattista 2013). The bars in all of these galaxies are also relatively smooth and, except for the sharp, right-angle-turning ansae of the bar in NGC 1042 (indicated by the $><$ symbols), are made largely of old stars.

The bars seen in Figure 18 can be contrasted with those shown in Figure 19. The top panels of Figure 19 show the deprojected $3.6 \mu \mathrm{m}$ images of NGC 600 and NGC 4731, types $\mathrm{SB}(\mathrm{rs})$ cd and $\mathrm{SB}(\mathrm{s}) \mathrm{d}$, respectively. In both cases, the bar is a line of star-forming knots (see also Martin et al. 1996). That such bars are likely to be highly flattened and confined to the thin disk is suggested by NGC 7090 and IC 1898 (Figure 19, middle), two nearly edge-on galaxies classified as type $\mathrm{SB}(\mathrm{s})$ dm $\mathrm{sp}$, where a distinct linear feature (whose extent is indicated by circumflexes) is seen in the inner regions. In both cases, subtle spiral structure (highlighted by horizontal dotted lines for IC 1898) breaks from near the ends of the linear feature, suggesting that the feature is a bar and not merely an inner disk.

The fact that these late-type galaxy bars show no inner 3D component and are defined to a signifcant extent by star formation indicates that the dichotomy in $f(F \geqslant 0.5)$ seen in

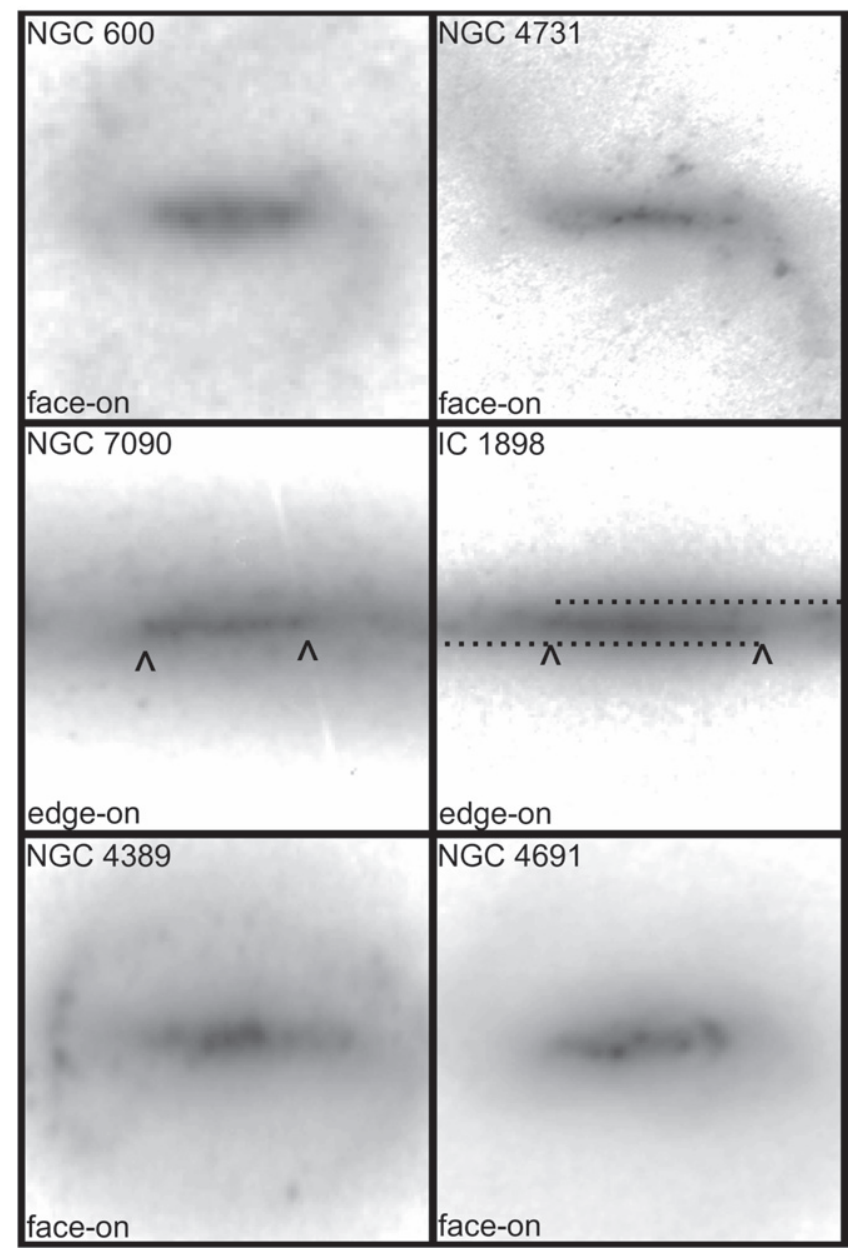

Figure 19. (Top row): deprojected $3.6 \mu \mathrm{m}$ images of 2 galaxies of mid-IR types Scd (NGC 600) and Sd (NGC 4731), rotated such that the bar is oriented horizontally. (Middle row): two edge-on galaxies showing a short, linear feature (indicated by the circumflexes) that is likely to be an edge-on view of the same kind of bar seen in NGC 600 and 4731. The dotted lines in the IC 1898 image highlight two spiral arms that break from the ends of the linear feature. The disk major axes for both galaxies are rotated to be horizontal. (Bottom row): deprojected images of two lower inclination early-type galaxies showing the same kind of bar seen in NGC 600 and 4731, rotated such that the bar is oriented horizontally. Units are mag $\operatorname{arcsec}^{-2}$.

Figure 6 reflects something more fundamental than a mere change in the bar fraction with galaxy types. The bars in Figure 18 are very different structures from those shown in Figure 19. While numerical simulations of the bar instability have had considerable success in explaining the structure of the types of bars seen in galaxies like NGC 1300 and 4314 (e.g., Athanassoula et al. 2014), the same is not true for the bars of NGC 600 and 4731 which have no 3D component, no X-pattern, and in general no ansae or obvious spiral character.

The lower two frames in Figure 19 show two Virgo Cluster galaxies whose stage is "early" but whose bar is clearly "late." NGC 4389 and NGC 4691 are classified in Table 6 as SB(rs)a [d] and $\left(\mathrm{R}^{\prime} \mathrm{L}, \mathrm{R}^{\prime}\right) \mathrm{SB}(\mathrm{s}) 0 / \mathrm{a}[\mathrm{dm}]$, respectively. The "B" in each case is a line of star-forming knots just as in NGC 600 and 4731. The disks, in contrast, are mostly devoid of star formation as would be found in S0/a or Sa galaxies.

The types of bars shown in Figure 18 are rarely, if ever, seen in galaxies of types Scd and later. Because these types of 
galaxies tend to have a lower average luminosity than S0/a to Sc types (e.g., dVA, figure 1.16), we may deduce that the fraction of Figure 18 bars drops precipitously for lower mass disk galaxies. We have excluded from the discussion the bars of Magellanic irregulars that have classifications such as IABm, $\mathrm{IB}(\mathrm{s}) \mathrm{m}$, etc. The reason is that the bars of such galaxies are often ill-defined, and the large spread in luminosity of Im galaxies means that many members of the class do not have the sophistication of structure needed to support the existence of a bar.

\subsubsection{Nuclear Rings, Lenses, and Secondary Bars}

Nuclear rings, lenses, and secondary bars are described in this section as features of the morphology of primary bars. All are dynamically important structures found in the centers of early-to-intermediate type barred galaxies (e.g., Buta \& Crocker 1993). Nuclear rings are especially heterogeneous in their morphological, metric, and star-forming characteristics. In some galaxies, a nuclear ring is the site of a starburst, and may be the only place in a galaxy where active star formation is occurring. In optical imaging, a nuclear lens may be interpreted as a quiescent nuclear ring in a non-star-forming phase, although not all nuclear lenses are necessarily dead nuclear rings.

Comerón et al. (2010) have provided the most extensive recent study of the morphology and metric characteristics of nuclear rings, and include not only star-forming and stellar features in the class, but also dust nuclear rings. The main requirement to be defined as a nuclear ring is proximity of the feature to the nucleus of a galaxy. Also, Comerón et al. (2010) required that the width of the ring is no more than half of its radius. As for inner and outer rings, there can be ambiguity in interpreting nuclear rings, even in some barred galaxies. These ambiguities are discussed in detail by Comerón et al. (2010).

Because the centers of intermediate-stage barred spirals can be affected by complex dust patterns, mid-IR imaging is especially useful for seeing the actual structure of nuclear rings, lenses, and secondary bars. Figure 20 shows the mid-IR morphology of the nuclear rings or lenses of $12 \mathrm{~S}^{4} \mathrm{G}$ galaxies. In spite of the emphasis on an older stellar population, the midIR morphology of nuclear rings can still display a knotty structure due to intense star formation.

In the CVRHS, nuclear rings are recognized with the symbol (nr). In some cases, an optical nuclear ring appears only as a subtle enhancement at the edge of a lens; these are recognized using the symbol (nrl). Nuclear lenses are recognized with the symbol (nl). Table 6 includes 38 galaxies classified as having an (nr), an (nrl), or a nuclear pseudoring $\mathrm{nr}^{\prime}$, and 33 galaxies as having an (nl). The metric characteristics of these features are provided in Comerón et al. (2010, 2014). Note that poor resolution can, in some cases, make a nuclear ring look more like a nuclear lens.

Most of the nuclear ring/lens features shown in Figure 20 are detectable as rings in blue light. Exceptions are the features seen in NGC 1365 and 5383, which appear as nuclear spirals carved by dust in blue light and more like rings in the mid-IR. Even the ring in NGC 1097 is much more spiral-like in blue light (dVA) compared to its very regular, almost circular shape in the mid-IR.

Secondary bars, also commonly known as nuclear bars, are well-known features of early-type barred galaxies (e.g., Laine et al. 2002; Erwin 2004). These small features often have a linear scale similar to nuclear rings. For example, NGC 5728 in Figure 20 shows a nuclear bar (oriented roughly horizontally) within its small nuclear ring (seen also in a NIRSOS $K_{s}$-image; Laurikainen et al. 2011). In the CVRHS, nuclear bars are recognized with the notation (nb). NGC 1291 (Figure 12) also shows a nuclear bar, but no nuclear ring surrounds this bar. The nuclear bar of NGC 1291 is actually a more prominent-looking feature than the galaxy's primary bar. Table 6 recognizes a definite or possible nuclear bar in 15 galaxies. There are undoubtedly many more present that would be detectable with better resolution.

\subsection{5. $X_{1}$ Rings}

$\mathrm{S}^{4} \mathrm{G}$ images provide an extinction-free view of a rare type of ring called an " $x_{1}$ ring." These are highly elongated rings which lie within and along a bar. The term $x_{1}$ refers to the main family of central orbits that support a bar (Contopoulos \& Grosbol 1989). Regan \& Teuben (2004) used numerical orbit calculations to show that inner rings in SB galaxies could be tied to looping 4:1 resonant orbits, and that if a bar is especially strong, shocks in the loops can collect gas into a highly elongated $x_{1}$ orbit within the bar. Star formation in this orbit would then form an " $x_{1}$ ring."

Only four possible examples of this type of feature are recognized in the $S^{4} G$ catalog (NGC 4569, 5334, 6012, and UGC 7848). The best example, NGC 6012, is shown in the left panel of Figure 21 (see also Regan \& Teuben 2004). The highly elongated, slightly miscentered feature extends to a little over half of the bar radius and is brighter at the ends. The galaxy also has a regular inner ring-lens $(\underline{r} \mathrm{l})$ and a very faint outer pseudoring.

NGC 5334 is an interesting late-type spiral that, in blue light, shows a bar that appears to have centered dust lanes (dVA). However, Figure 21 shows that the the split character of the bar is not due to dust, but to a highly elongated ring-like morphology. This is similar to NGC 6012 and we interpret the feature as another $x_{1}$ ring.

\subsection{The Mid-IR Morphology of Galactic Disks}

The considerable depth and dust penetration of $3.6 \mu \mathrm{m} \mathrm{S}{ }^{4} \mathrm{G}$ images provides excellent views of galaxy disks, especially thin disks which may be obscured by planar dust in optical images. In this section, we examine the mid-IR morphology of highly inclined and edge-on disks, focussing on warps, embedded disks in 3D systems, thick disks, and other special cases.

\subsubsection{Warped and Flared Disks}

Warped disks are easily recognizable in many $\mathrm{S}^{4} \mathrm{G}$ galaxies (see also Saha et al. 2009). Warping can appear as an edge-on disk twisted into an integral sign shape. The inner thin disk may be perfectly flat, while the outer parts of the disk bend in opposite senses from one end of the major axis to the other. Kinematically, a warp can be described in terms of circular orbits having a radius-dependent inclination and line of nodes position angle (e.g., Briggs 1990).

Since warps are most easily recognized in edge-on galaxies that would be classified as spindles ("sp"), the CVRHS recognizes warps using the notation "spw." Disk warping may be caused by a bending instability (Binney \& Tremaine 2008), among other possible explanations (e.g., Radburn-Smith et al. 

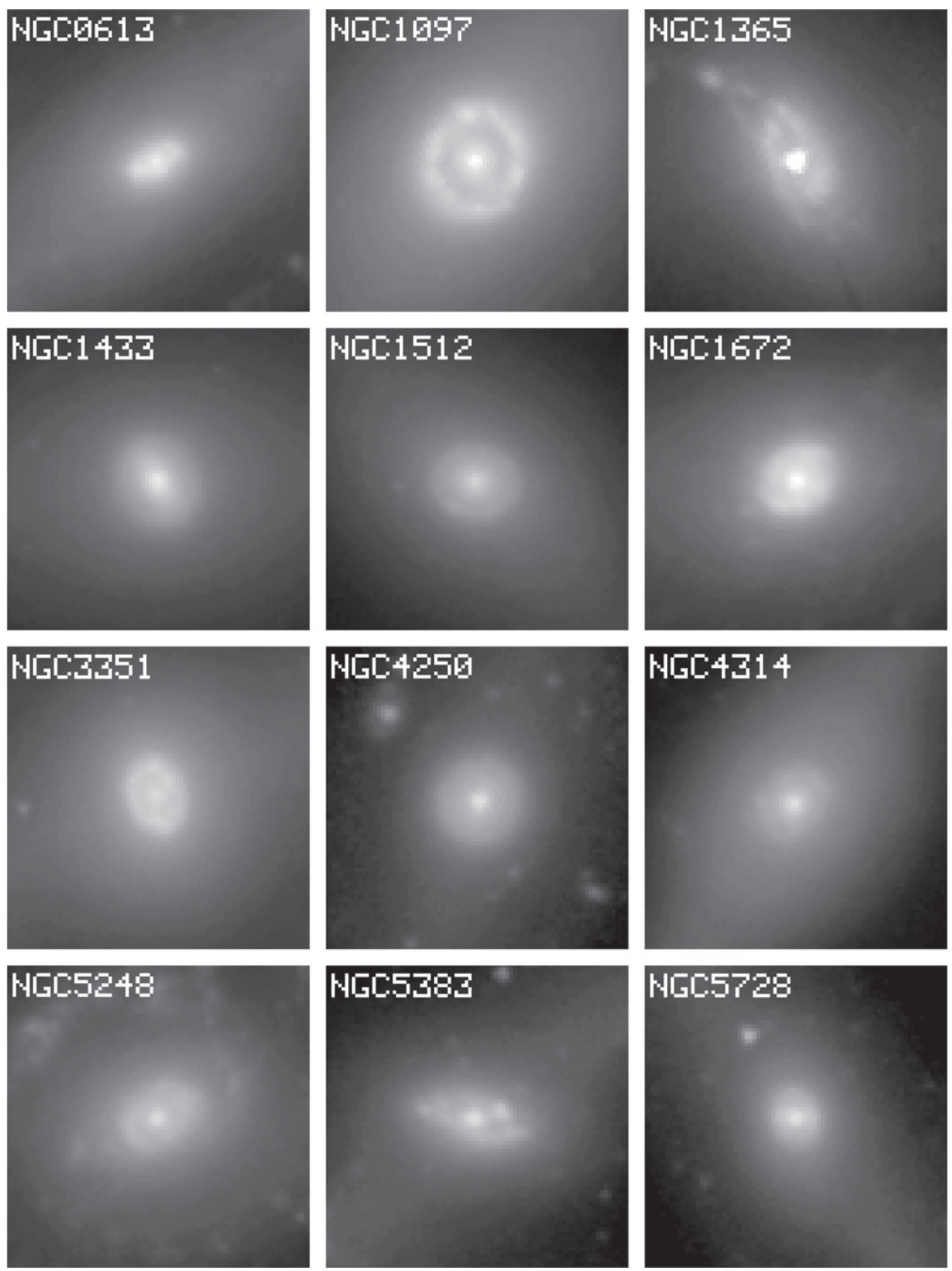

Figure 20. Montage showing the mid-IR morphology of the nuclear rings and lenses in $12 \mathrm{~S}^{4} \mathrm{G}$ galaxies.
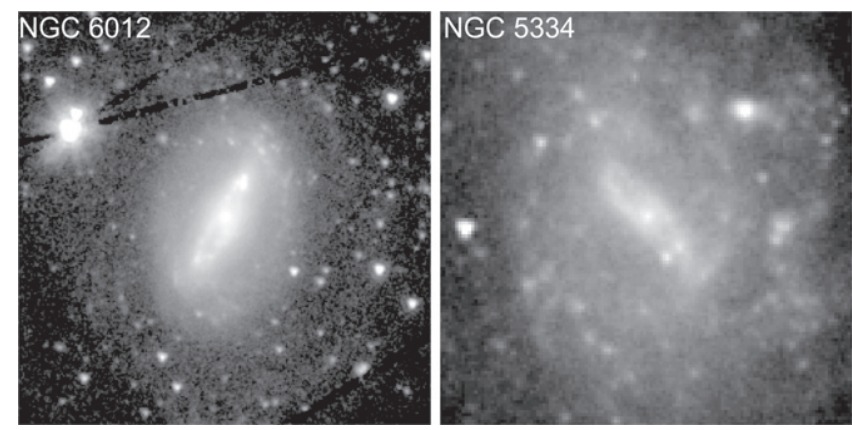

Figure 21. Two $S^{4} G$ galaxies showing $x_{1}$ rings, which are the highly elongated features in the bar.
2014). An up-to-date review of the theory of warps is presented by Sellwood (2013).

Four $\mathrm{S}^{4} \mathrm{G}$ galaxies having obvious warps are shown in Figure 22. These are selected merely as good examples that are well-resolved in $\mathrm{S}^{4} \mathrm{G}$ images. NGC 522 is an edge-on galaxy whose mid-IR thick disk structure has been studied by Comerón et al. (2011a). The galaxy is classified as type Sbc in RC3, but in the mid-IR it appears almost smooth enough to be classified as type S0. A subtle knottiness along the thin disk component favors a later type. The image shows strong warping and a subtle $\mathrm{X}$ in the central area that may signify the presence of a bar.

NGC 5084 shows a thin disk that flares within the prominent bulge region, and then bends farther out. No planar dust is 


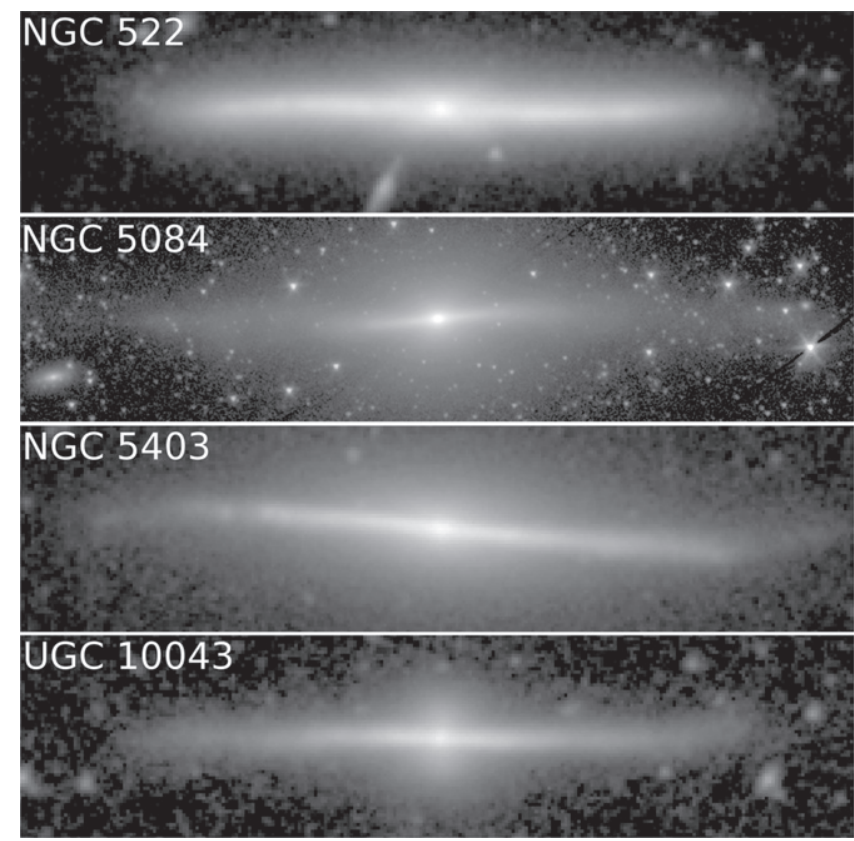

Figure 22. Montage showing the mid-IR morphology of four $S^{4} G$ galaxies having strong outer disk warping.

present in the thin disk as seen in an SDSS color image, and thus optical and mid-IR images provide similar views of the galaxy's structure.

NGC 5403 shows a bright thin disk embedded within a flattened spheroidal component. The thin disk bends and flares at large radii. An SDSS color image shows that the galaxy has a strong planar dust lane and is likely almost exactly edge-on.

UGC 10043 is an exceptional example with strong warping and a central bulge with an interesting peculiarity: inside the roundish region, the isophotes are elongated perpendicular to the inner disk. The appearance suggests that the inner part of the bulge has a prolate shape. This galaxy was recently studied in detail by Matthews \& de Grijs (2004), who concluded the galaxy may have experienced an accretion or merger event that can account for some of its unusual characteristics, such as the warped disk.

\subsubsection{Embedded Disks in Three-dimensional Early-type Galaxies}

The $\mathrm{S}^{4} \mathrm{G}$ sample includes numerous examples where a clear highly flattened disk-shaped system is embedded in a more three-dimensional early-type system. Such systems have been known for a long time, and highlight how some disks appear to be "living within an elliptical" (or how some bulges are "ellipticals living within a disk" e.g., Kormendy \& Kennicutt 2004). The mid-IR provides a dust-penetrated view of these interesting systems that highlights how thin disks can fill, overextend, or underextend the 3D systems they are embedded within.

Figure 23 shows a sampling of examples of these kinds of cases beginning with NGC 3377, a normal "disky elliptical" galaxy (type E(d)4, Kormendy \& Bender 1996). The disk in NGC 3377 is very subtle, featureless, purely stellar in nature, and possibly not exactly edge-on. The presence of the disk was quantitatively established by Jedrzejewski (1987), who showed that the $\cos 4 \theta$ relative Fourier deviation from perfect ellipses is positive across a wide range of radii in this galaxy.
NGC 681 is very similar to NGC 3377, except that the disk appears to be a nearly edge-on late-type spiral. The published classifications of NGC 681: SAB(s)ab sp (RC3) and Sab (Sandage \& Tammann 1981) seem inadequate to describe this galaxy. It is not clear that NGC 681 is merely an early-type spiral galaxy with a large bulge. The disk part of NGC 681 is a clear late-type spiral with a high degree of knottiness and star formation. The logarithmic, background-subtracted display shows that the embedded disk neither greatly overfills nor underfills the 3D component, but fades to a similar extent.

In Table 6, embedded disk systems such as NGC 681 are classified generally in a two-part manner. The 3D early-type component is usually denoted with " $E$ " because of appearance only, not because these components necessarily have $r^{\frac{1}{4}}$ luminosity profiles. For example, NGC 681 is classified in Table 6 as SA:(s:) $\underline{b}$ c sp/E(d)3, where the $E(d) 3$ refers to the 3D component as a "disky elliptical" (using Kormendy \& Bender 1996 notation). The adopted classification in Table 6 recognizes both the similarity to NGC 3377 and the greater significance of the embedded disk.

Very similar to NGC 681 is NGC 3683, whose disk is less edge-on and has a somewhat smaller bulge. It also is embedded in an E3 background. The Phase 1 and 2 classifications in Table 2 disagree on the presence of a bar in this galaxy; the mean classification of $\mathrm{SAB}(\mathrm{s}) \mathrm{b} \underline{\mathrm{c}} \mathrm{sp} / \mathrm{E} 3$ is an average of $\mathrm{SA}$ and SB. NGC 5078 is another example, but the embedded disk is more edge-on; its average type is $\mathrm{S}(\mathrm{r}:) \mathrm{b}$ sp/E5. The lack of a "(d)" in these two cases (as in E(d)5) only means that the outer isophotes of the E5 part are neither pointy nor boxy as judged visually in a color display, although the inner isophotes would be disky.

Related to these is the remarkable case of NGC 3675, where the embedded disk is far from edge-on and its structure is clearly visible. The Phase 1 classification recognizes two outer pseudorings, but only the inner one is well-defined and was also recognized in Phase 2. The final classification is $\left(\mathrm{R}^{\prime}, \mathrm{R}^{\prime}\right) \mathrm{SA}$ (ls)b/E4, recognizing an inner lens with a faint spiral pattern. The main outer pseudoring is about twice the diameter of the inner lens and bright spiral structure breaks from it to larger radii, forming another outer pseudoring. The diskiness of the E4 isophotes is not evident because the disk inclination is so low.

Like NGC 681, NGC 3683 and 5078 are cases of comparable extent embedded disks, where the disk appears to nearly fill the detectable outer isophotes of the 3D component. Also shown in Figure 23 are cases of what we will call "limited extent disks," where an edge-on disk appears to fade well within the bright bounds of the 3D component. An excellent example is NGC 4370, where the embedded disk is so small it was classified as a nuclear disk in Phase 2. Phase 1 also recognized boxy outer isophotes of the $3 \mathrm{D}$ component. The adopted average classification is $\mathrm{S}^{-10} \mathrm{sp} / \mathrm{E}(\mathrm{b}, \mathrm{nd}) 4$. An interesting aspect of NGC 4370 is the slight misalignment between the inner disk and the outer, boxy isophotes. A galaxy similar to NGC 4370 is described by Graham et al. (2012).

NGC 3115 is the well-known example originally classified by Hubble (1936) as type E7. It was reclassified as $\mathrm{SO}^{-} \mathrm{sp}$ in RC3 and as $\mathrm{S}_{1}(7) / \mathrm{a}$ by Sandage \& Bedke (1994). Although the morphology of NGC 3115 in the mid-IR hardly differs from its morphology in blue light, it is a good example to compare with the other embedded disks illustrated in Figure 23. The bright thin disk appears to fade well before it fills the major axis 

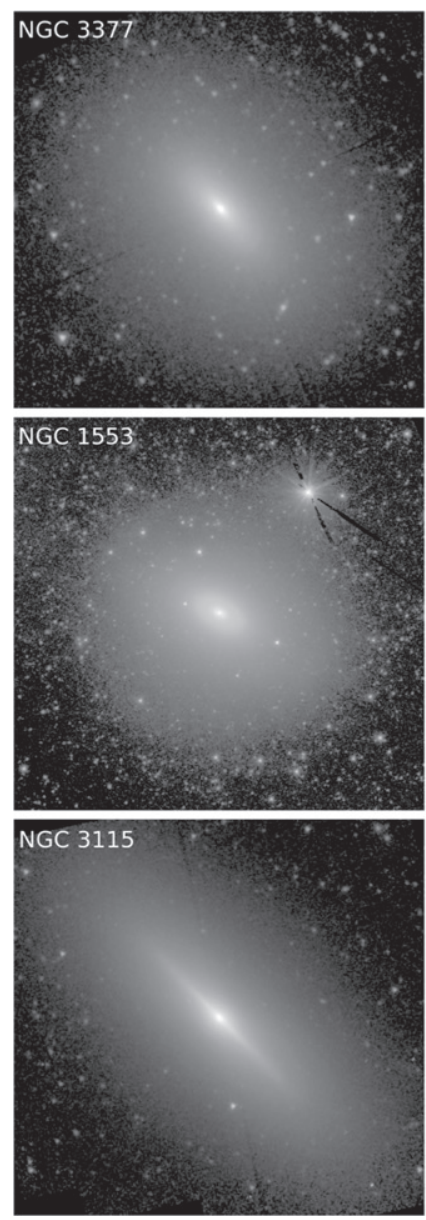
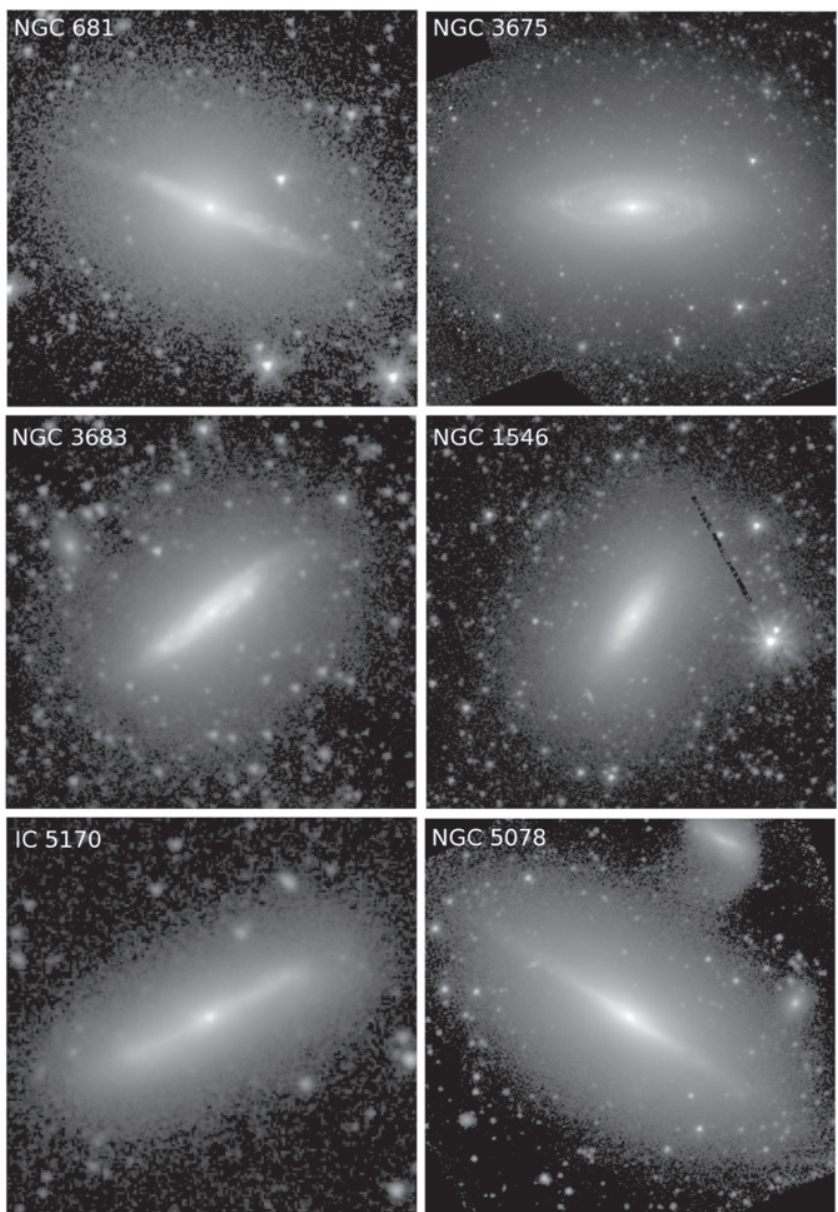
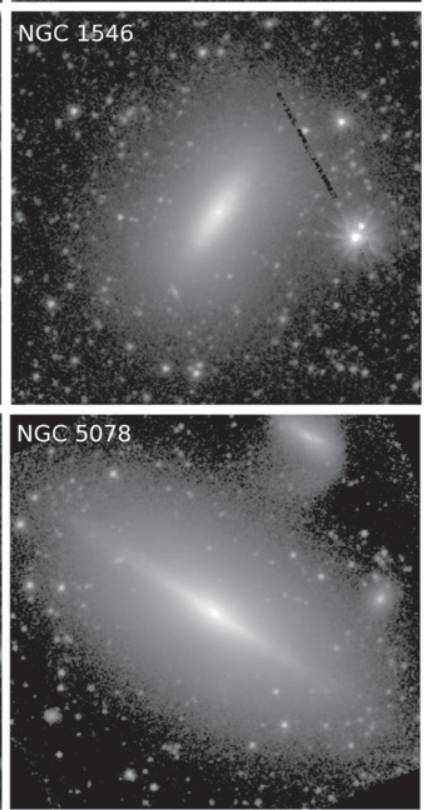

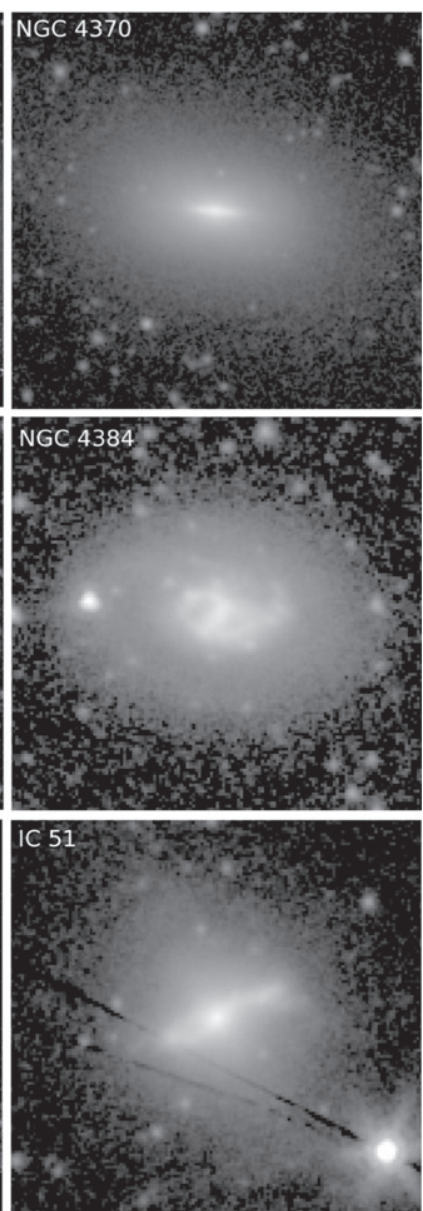

Figure 23. Montage showing galaxies having embedded disks in 3D early-type systems.

of the 3D component. In this case the outer isophotes of the 3D component are not obviously disky, and the classification adopted in Table 6 is $\mathrm{SO}^{-}$sp/E5-6.

NGC 1546 is an example where a non-edge-on embedded disk includes a lightly patchy pair of closely spaced rings. The outer isophotes of the 3D component are slightly boxy. This was recognized in both the phase 1 and 2 classifications, and the average classification is $\left(\mathrm{R}^{\prime}\right) \mathrm{SA}(\mathrm{r}) \mathrm{a} / \mathrm{E}(\mathrm{b}) 3-4$. We have already noted a similar embedded disk ring/lens in NGC 1553.

IC 5170 is a case where what appears to be an abruptly terminating edge-on disk is embedded within a boxy 3D component. The mean Phase 1 and 2 type is $\mathrm{S} 0 / \underline{\mathrm{a}}$ spw/E(b)5. NGC 4634 (Figure 24) shows a similar sharply ending limited extent thin disk embedded in a larger, boxy zone.

IC 51 is a very unusual case where the embedded disk is not settled within the projected major axis of the rounder background component. In this case, the background component could be a more face-on disk, and IC 51 would be an example of a polar ring galaxy where only the disrupted disk is seen edge-on. The Table 6 classification is $\mathrm{SA}(\mathrm{s}) \mathrm{b} \mathrm{sp} / \mathrm{SA} 0^{\circ}$ (PRG?). IC 51 is also listed as a "good candidate" for a polar ring galaxy by Whitmore et al. (1990).

The final example in Figure 23 is NGC 4384. The inner part of the galaxy is a clear $\mathrm{SB}(\mathrm{rs}) \mathrm{dm}$ type with virtually no bulge. This appears embedded in a smooth, relatively uniform background interpreted in both Phase 1 and Phase 2 as an outer lens $(\mathrm{L})$.

\subsubsection{Thick Disks}

Thick disks are an important morphological feature in many $S^{4} \mathrm{G}$ galaxies, especially among late-type systems with little bulge contribution. A thick disk is defined to be a highly flattened component with a vertical scale height a few times larger than that of the thin disk (Comerón et al. 2011a). Because of the considerable depth of $\mathrm{S}^{4} \mathrm{G}$ images and the almost negligible impact of extinction, thick disks are very prominent and are especially made visible with the logarithmic display that we use. $S^{4} \mathrm{G}$ images allow us to see the relation between thick and thin disks only minimally affected by extinction of the thin component.

Figure 24 shows five $\mathrm{S}^{4} \mathrm{G}$ galaxies having well-defined but typical thick disks. The features have a range of morphologies. For example, the thick disks of NGC 5470, NGC 4217, and UGC 7522 are pointed ovals at the faintest light levels detected, which may not be unusual except for the fact that some thick disks are either more elliptical than pointy at the ends (as in IC 2135 bottom frame, Figure 24) or are even boxy (as in NGC 4634 middle frame, Figure 24). Each galaxy in Figure 24 has a two part classification as described in Section 3.3. The thick disk is treated as a highly elongated disky, boxy, or plane elliptical feature using Kormendy \& Bender (1996) notation.

Notes on several of the galaxies in Figure 24:

NGC 4217 is classified as an edge-on Sb spiral in RC3, but the $3.6 \mu \mathrm{m}$ image shows only a small central concentration, more characteristic of $\mathrm{Sc}$ than $\mathrm{Sb}$. The star-forming disk is very 

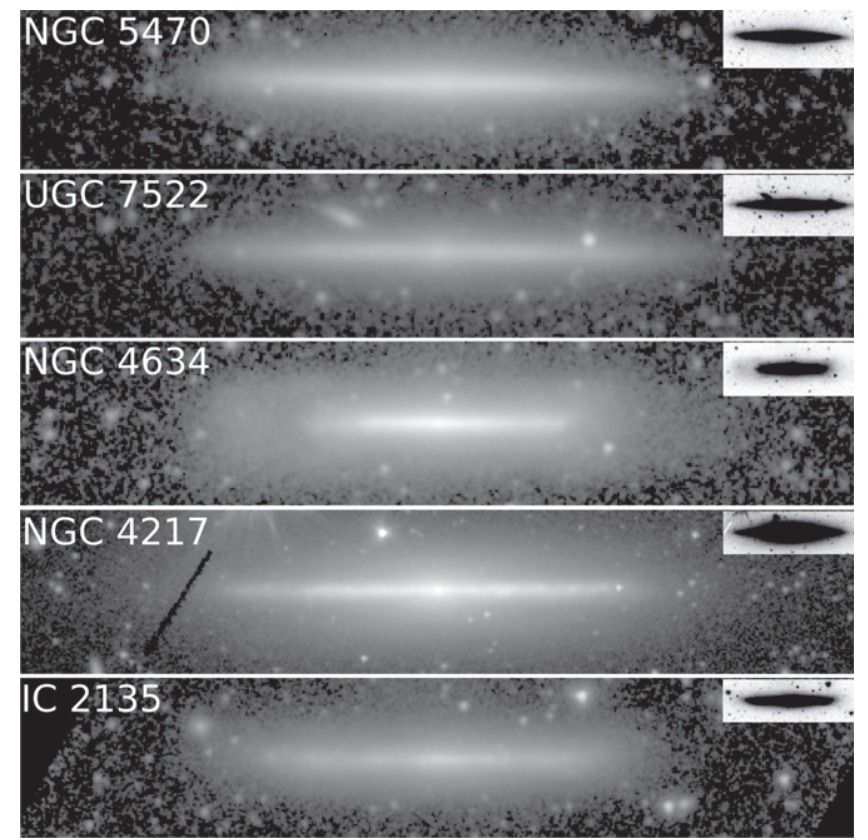

Figure 24. Montage showing five $\mathrm{S}^{4} \mathrm{G}$ galaxies having typical thick disks.

thin, and is embedded in a thick disk. The galaxy appears to be an Sc embedded in an E6-7 thick disk (Table 6 classification: Sc $\mathrm{sp} / \mathrm{E}(\mathrm{d}) 6-7)$, and is exactly edge-on. The inserts in Figure 24 for NGC 4217 show how the shape of the fainter isophotes changes with decreasing surface brightness.

NGC 5470 is an almost exactly edge-on disk galaxy also classified as type $\mathrm{Sb}$ in $\mathrm{RC} 3$. In the mid-IR, however, NGC 5470 is a completely bulgeless, pure disk galaxy that shows an S0-like appearance. The Table 6 classification, $\mathrm{S} 0^{\circ}[\mathrm{d}] \mathrm{sp} / \mathrm{E}(\mathrm{d}) 8$, alludes to the van den Bergh (1976) parallel sequence idea, although genuine $B$-band versions of such a galaxy type are not necessarily known. It is possible that NGC 5470 would be less S0-like in a higher resolution mid-IR image. The $E(d) 8$ part of the classification strictly recognizes the highly flattened thick disk with a strong pointed oval shape.

NGC 4634 is a member of the Virgo Cluster and shows a thin planar dust lane in blue light. The boxiness of its thick disk could be an environmentally driven product. Kormendy \& Bender (2012) have interpreted the broad boxy zone outside the edge-on S0 galaxy NGC 4638, also a Virgo Cluster member, as possibly being a thick disk environmentally flared by harassment from cluster encounters. NGC 4634 is classified in Table 6 as $\mathrm{Sd}$ sp/E(b)7-8. Like NGC 5470, the $3.6 \mu \mathrm{m}$ image of NGC 4634 shows little or no central concentration. Also, the thin disk in this case has limited extent.

\subsubsection{Extraplanar Disks}

An extraplanar disk in a galaxy is an acquired disk where the material lies in a different plane from the disk of the receiving galaxy. The best-known extrapalanar disks are polar rings, where a small gas-rich companion is disrupted into a polar orbit around a more massive S0 galaxy (Schweizer et al. 1983). An inclined ring is an extraplanar disk where a companion has been disrupted along a lower inclination orbit. Inclined and polar ring galaxies are most easily recognized when both the receiving disk and the extraplanar disk are nearly edge-on (Whitmore et al. 1990).
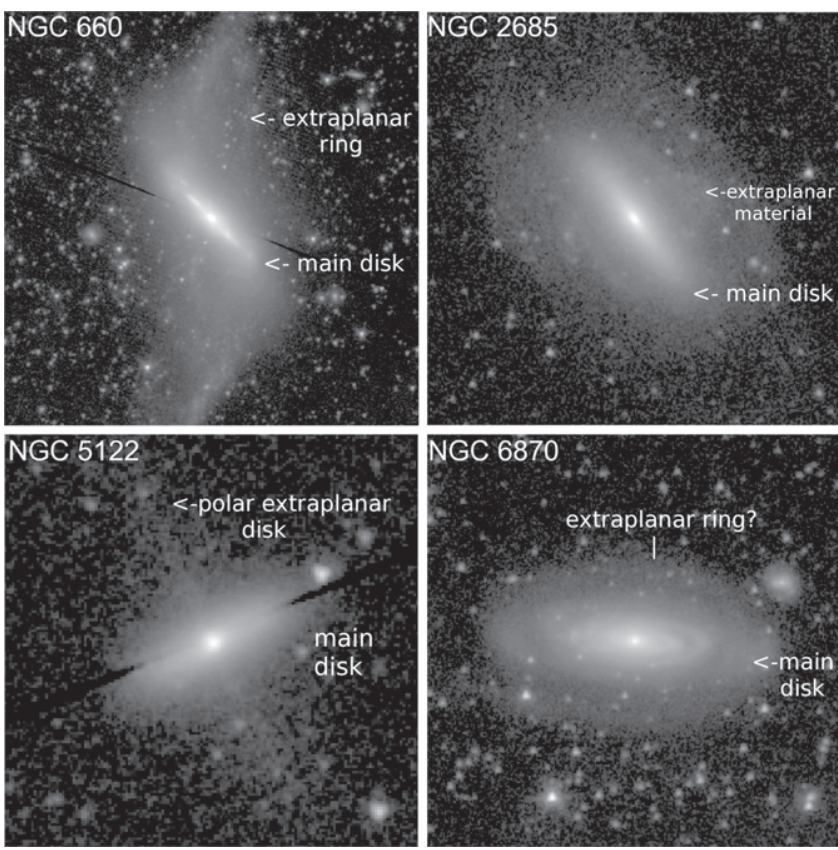

Figure 25. Montage showing four $S^{4} G$ galaxies having definite or possible extraplanar disk material. The main (receiving) disk and the extraplanar disk are indicated.

Galaxies showing extraplanar material in the form of an inclined disk or ring have been of great interest for studies of disruptive encounters, merger histories, and the shapes of dark matter halos (e.g., Casertano et al. 1991, and most recently Iodice \& Corsini 2013). Several $S^{4} G$ galaxies show definite or possible extraplanar disk material. Four examples are shown in Figure 25. Three of these (NGC 660, 2685, and 5122) were already known from optical observations, and we have already described IC 51. Whitmore et al. (1990) show how detection of polar ring galaxies, where the extraplanar disk is oriented at nearly $90^{\circ}$ to the main disk (typically an S0 galaxy), depends strongly on viewing geometry.

NGC 660 is interesting in that in blue light, the main disk shows an aligned dust lane that is at an angle to a second dust lane. It was classified as a polar-ring galaxy (PRG) in Phase 1 (paper I), but here we prefer the term "inclined ring galaxy" (IRG) since the extraplanar material in cases like NGC 660 is not necessarily oriented orthogonally to the main disk (van Driel et al. 1995). The $S^{4} G 3.6 \mu \mathrm{m}$ image of NGC 660 reveals an interesting characteristic: a broad $\mathrm{X}$ pattern flanked by two bright ansae. This indicates that the main component of NGC 660 has a bar (see also Luetticke et al. 2004).

The well-known extraplanar material in NGC 2685 is seen in the $3.6 \mu \mathrm{m}$ image as a small tipped partial ring of knots. This has been interpreted as part of a ring at a large angle to the main stellar object. Józsa et al. (2009) argue that there are indeed two disks of different orientations in NGC 2685, but the second disk (which has the bulk of the HI) is extremely warped in the inner regions and appears extraplanar as a result. NGC 2685 is likely not a classical polar ring galaxy.

NGC 5122 (lower left panel of Figure 25) shows a clear edge-on polar disk and is the best example of a polar ring galaxy in the catalog. Whitmore et al. (1990) argue that for every case like this in a sample, there should be a few where the orientation of the two disks is far enough from edge-on that the 


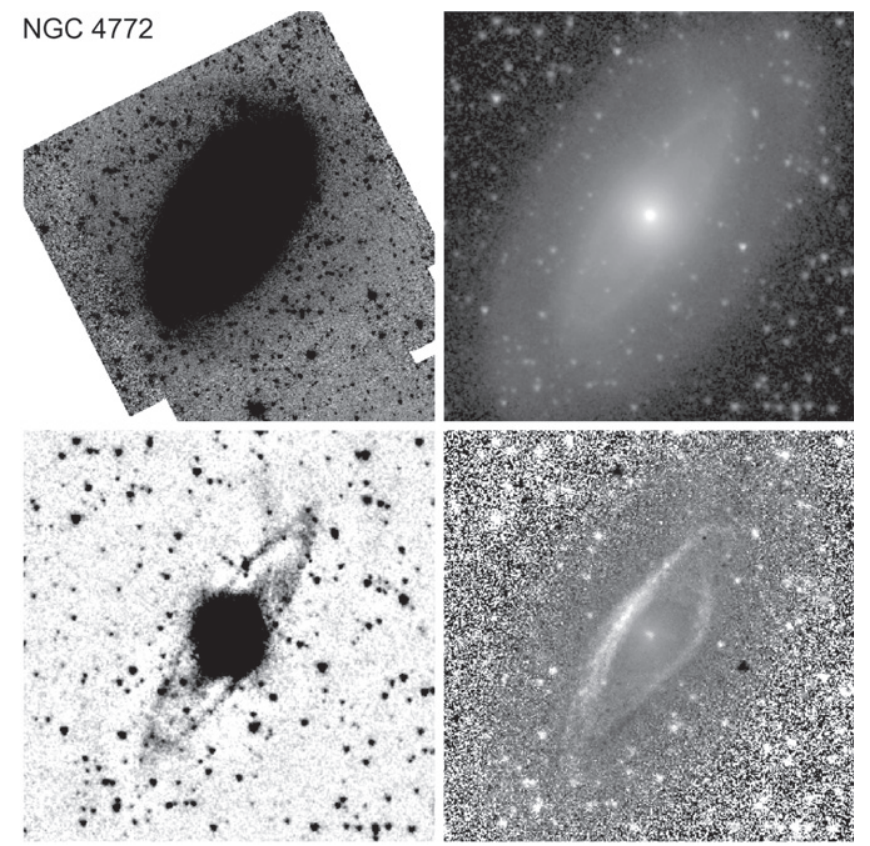

Figure 26. A look at $S^{4} \mathrm{G}$ galaxy NGC 4772, a candidate for a masquerading inclined ring galaxy: (Top left)—frame showing the very faint outer disk light that is round enough to suggest an inclination of less than $40^{\circ}$; (Upper right) the structure in the overexposed inner oval zone is shown at twice the scale this reveals the highly elongated inner ring. Lower left-this frame is an unsharp-masked version of the upper right frame showing how thin and welldefined the inner ring is; lower right - a $g-[3.6]$ color index map of NGC 4772 coded such that blue features are dark and red features are light. This shows the complex dust and near-side extinction in the region of the inner ring. The scale of the last three frames is twice that of the upper left frame. The lower left frame is in intensity units; the other frames are in units of mag $\operatorname{arcsec}^{-2}$.

object could masquerade as a relatively normal-looking system. NGC 6870, shown in Figure 25, could be such a case. The galaxy shows a bright inner, highly tilted (but not edge-on) disk that seems to have less inclined material crossing just inside the ends of the inner disk, giving the galaxy the subtle appearance of a hat.

NGC 4772 (Figure 26) is a galaxy that, in blue light, shows a bright bulge in the center of an odd ring with strong near-side extinction. (This can be seen in the lower right panel of Figure 26, which shows a $g-[3.6]$ color index map of the galaxy.) The extinction suggests a substantial inclination, but the faintest outer $3.6 \mu \mathrm{m}$ isophotes of the galaxy (shown in the upper left frame of Figure 26) are much rounder than the ring and imply an inclination of less than $40^{\circ}$. This outer light has the morphology of an $\mathrm{R}_{1}{ }^{\prime}$ outer pseudoring relative to a broad oval which is shown at twice the scale in the upper right panel of Figure 26. An unsharp-masked version of this image shows a very thin, regular ring with no hint of excess star formation around its major axis. If the ring were coplanar with the broad oval, it would likely show such an excess (e.g., Crocker et al. 1996). The implication is that the $\mathrm{R}_{1}{ }^{\prime}$ outer pseudoring and the larger oval are part of one disk, while the inner ring could be part of a second disk that is not coplanar with the first one. The bulge in NGC 4772 is significant and largely spherical, making it unlikely that the inner ring is intrinsically oval.

As noted by Whitmore et al. (1990), the only way to prove that a specific case is a genuine PRG or IRG is to make kinematic observations. Haynes et al. (2000) observed NGC
4772 in $\mathrm{HI}$ and found that the galaxy has two somewhat kinematically decoupled $\mathrm{HI}$ rings of different shapes and position angles (associated with the $\mathrm{R}_{1}{ }^{\prime}$ outer pseudoring and the inner ring). From the velocity field, these authors concluded that the galaxy has suffered a minor merger.

NGC 4772 demonstrates how comparisons between optical and mid-IR images can lead to candidates for extraplanar disks that we know have to exist, but which are overlooked due to unfavorable orientations of the disks.

\subsection{Late-type and Spheroidal Galaxies}

The Virgo Cluster is well-represented in the $S^{4} G$ database. Included in the sample are objects that were classified by Binggeli et al. (1985) as dwarf elliptical (dE) and dwarf S0 (dS0) galaxies. Kormendy (2012) reviews the evidence that $\mathrm{dE}$ and dS0 galaxies are environmentally modified, bulgeless extreme late-type galaxies whose existence favors reconsideration of the old van den Bergh (1976) "parallel sequence" idea (see also Cappellari et al. 2011; Laurikainen et al. 2011). For historical reasons, Kormendy collectively calls these objects "spheroidals" (Sph), although the term is not meant to imply anything about intrinsic shapes. This conclusion is based on effective parameter correlations (effective surface brightness $\mu_{e}$ and effective radius $r_{e}$ versus absolute $V$-band magnitude, for example) which revealed that $\mathrm{dE}$ and $\mathrm{dS} 0$ galaxies occupy areas where irregular galaxies and very late-type spirals are found. Genuine dwarf ellipticals, like M32, are much rarer than Sph galaxies, and the $S^{4} \mathrm{G}$ sample, in fact, includes no genuine dwarf elliptical galaxies.

The connection between irregulars and $\mathrm{Sph}$ galaxies seems evident in $\mathrm{S}^{4} \mathrm{G}$ images. Because the effects of star formation are reduced, a $B$-band irregular galaxy can look like a $\mathrm{dE}$ or $\mathrm{dS} 0$ galaxy at $3.6 \mu \mathrm{m}$. An example is NGC 3738, shown in Figure 27. In Table 6, the galaxy is classified as $\mathrm{dIm}(\mathrm{dE}) / \mathrm{Sph}$, indicating it has a bright background that resembles a $\mathrm{dE}$ galaxy. Such a galaxy, if environmentally modified, would probably look very much like a Virgo Cluster dE or dS0. We use "/Sph" to indicate a likely connection. Figure 27 shows several other examples. NGC 4328 shows a clear inner lens but no bar, while NGC 4506 shows a very faint, low luminosity spiral, embedded in both cases in an Sph background.

Kormendy (2012) also shows that higher-luminosity Sph galaxies are bulgeless S0s. Two galaxies likely to be related to these are NGC 4064 and 4312 (Figure 27). NGC 4312 is a definite member of the Virgo Cluster while NGC 4064 is a likely member. Both appear highly inclined and show a narrower elongation in the inner regions that could be interpreted as a bar. The appearance of these galaxies at $3.6 \mu \mathrm{m}$ favors an early CVRHS stage, yet both appear largely bulgeless. These could be environmentally modified late-type galaxies. This result is similar to what was found by Laurikainen et al. (2010), where some SOs from the NIRSOS were shown to have very small bulges and were presented as evidence in support of the parallel sequence classification.

Figure 28 shows four similar low-luminosity non-Virgo Cluster members, each of which has a similar projected outer isophotal shape and a narrower brightness enhancement along the major axis. In NGC 4248, the isophotal differences between the inner and outer regions seem obvious; the same is true for ESO 419-13. In both cases, the inner elongation is parallel to the background major axis. While the inner zones could be bars, another interpretation is that these galaxies are edge-on Im 

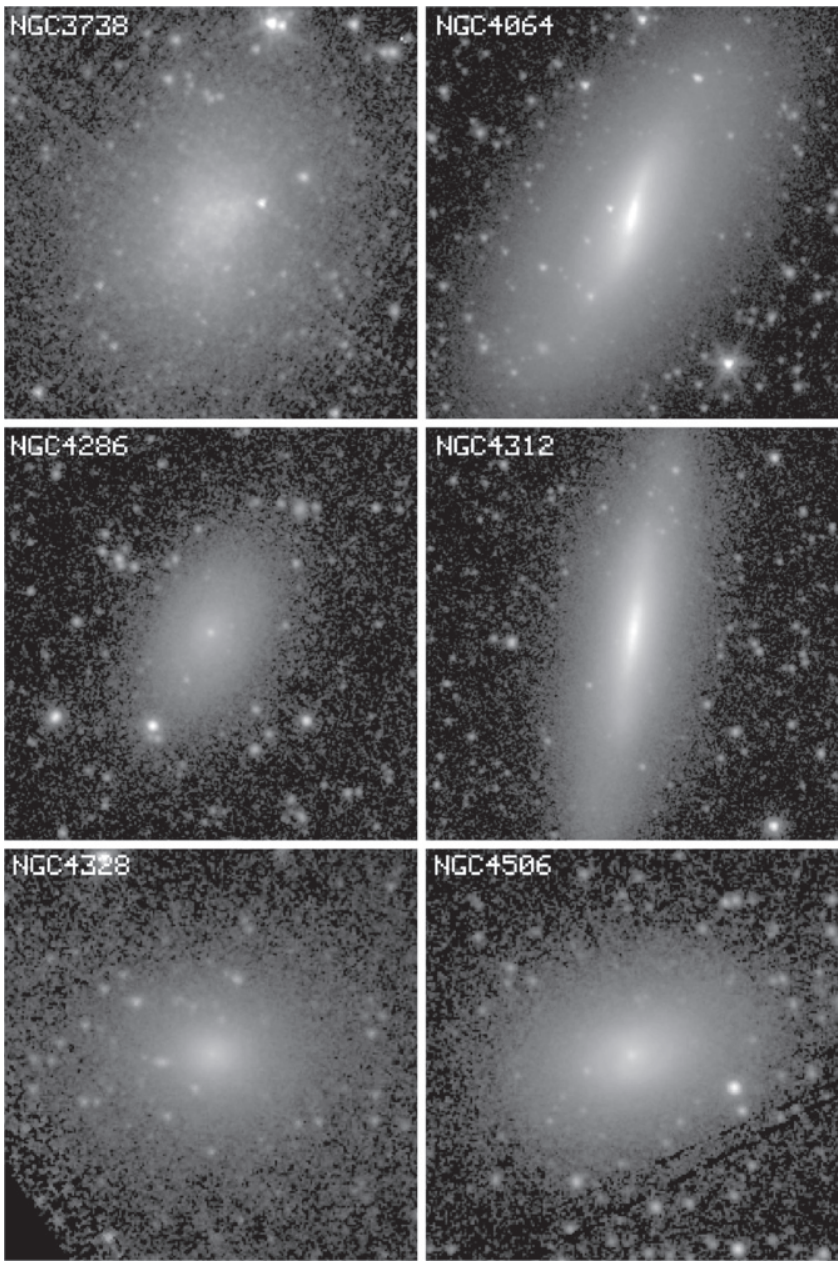

Figure 27. Six examples of Kormendy spheroidal galaxies, including two (NGC 4064 and 4312) that could be interpreted as bulgeless S0 galaxies in the mid-IR.

galaxies embedded in a 3D background Sph. ESO 357-25 and ESO 359-29 appear related to these two objects but the central "stripe" is less well-defined.

Kormendy's conclusion concerning dE and dS0 galaxies has been challenged by Graham (2013 and references therein) as being due to the misleading nature of nonlinear parameter correlations. Interpreted in a different way, regular ellipticals and $\mathrm{dE}$ and $\mathrm{dS} 0$ galaxies do not necessarily divert from a smooth connection to more luminous ellipticals.

\subsection{The Mid-IR Morphology of Spiral Arms}

The morphology of spiral structure in the mid-IR is of great interest for several reasons: (1) the mid-IR reveals the underlying stellar density enhancements associated with the arms, allowing a more reliable judgment of the significance of the spiral to the overall dynamics and evolution of a given disk galaxy; (2) the minimally extinguished view of spiral arms provided by mid-IR imaging allows not only the true character of the arms (such as pitch angle and relative amplitude) to be meaured, but also all star-forming regions associated with the arms will be seen, including those which may have been heavily obscured; and (3) like the classification of bars, the classification of spiral arms in the mid-IR should be more reliable than in blue light.

In this section, we examine the morphology of spiral arms using arm classifications that are independent of CVRHS classifications, and also look more closely at a few examples of extreme spiral structure in the $\mathrm{S}^{4} \mathrm{G}$.

\subsubsection{Arm Classifications}

Arm classifications for galaxies are based on the symmetry, continuity, and number of spiral arms. Arm Classes are distinct from the Hubble and de Vaucouleurs classifications in that they are independent of the pitch angle or bulge/disk ratio. Elmegreen (1981) introduced the term "flocculent" to describe galaxies with short spiral arm pieces, in contrast to the Lin \& Shu (1966) grand design spirals with long symmetric spiral arms. Multiple arm galaxies fall in between these extremes but are more similar to grand design galaxies, with inner two-arm symmetry branching to many long arms. Elmegreen \& Elmegreen (1982) devised a 12-point classification system that examined nuances of these structures. Arm classes from $B$ band images were determined for over 700 galaxies based on the Palomar Observatory Sky Survey and high resolution atlas images (Elmegreen \& Elmegreen 1987).

The physical nature of flocculent, multiple arm, and grand design galaxies is revealed through a comparison of blue and near-IR (I-band) spiral arm strengths, in which the contrast between arm and interarm regions is essentially the same in the $B$ - and $I$-bands for grand design and multiple arm galaxies but not for flocculent galaxies. Also, the arm amplitude is stronger in grand design galaxies (Elmegreen \& Elmegreen 1984). The underlying mechanism that explains global symmetry in the grand design and multiple arm galaxies is a density wave, which flocculent galaxies appear to lack in blue light.

Previous studies of $2 \mu \mathrm{m}$ images, which like IRAC images are less affected by dust extinction than optical images, showed that sometimes an "optically flocculent" spiral pattern looked grand design in the near-IR. Four examples of this, including NGC 5055, were studied by Thornley (1996), and NGC 253 and 7217 are two more examples described in the dVA. Paper I compared $B$-band and $3.6 \mu \mathrm{m}$ images of the well-known optically flocculent spiral NGC 2841, showing that it also has an underlying stellar grand design spiral.

To help elucidate whether underlying disks might contain an obscured density wave pattern, arm classifications were extended to $3.6 \mu \mathrm{m}$ by Elmegreen et al. (2011) in $46 \mathrm{~S}^{4} \mathrm{G}$ galaxies; most did not change their Arm Class going from $B$ band to $3.6 \mu \mathrm{m}$. Spiral arm properties were studied through measurements of symmetric components, arm-interarm amplitudes, and Fourier transforms. As for the $B$-band images, the $3.6 \mu \mathrm{m}$ images showed stronger Fourier components and stronger arm amplitudes in grand design and multiple arm galaxies than in flocculent galaxies.

Arm classifications are now extended to the full sample of $\mathrm{S}^{4} \mathrm{G}$ galaxies in Table 9, where flocculent, multi-arm, and grand design morphologies are symbolized using F, M, and G, respectively. These classifications are given only for $1114 \mathrm{~S}^{4} \mathrm{G}$ spiral galaxies that are not too inclined to make the arm class indistinguishable, and are listed also in the notes to Table 6. The classifiable subsample contains $50 \%$ flocculent, $32 \%$ multi-arm, and $18 \%$ grand design cases. While flocculent galaxies tend to be late-type, the arm classes span the range of CVRHS spiral stages. Of these galaxies, 317 are in common 

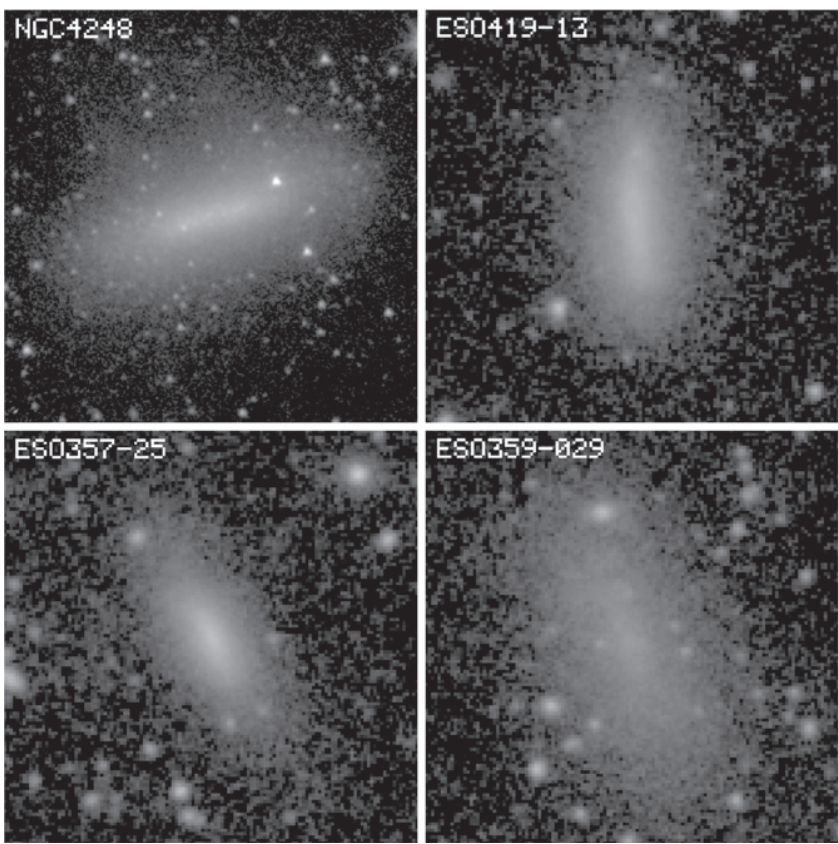

Figure 28. Four low-luminosity, likely Sph galaxies showing an inner zone that is more elongated than the outer isophotes parallel to the major axis. None is a member of the Virgo Cluster.

with galaxies for which a $B$-band arm class was previously published. A comparison of the classifications showed that $60 \%$ of the galaxies have the same Arm Class going from $B$ band to $3.6 \mu \mathrm{m}, 28 \%$ changed from flocculent to multi-arm or multi-arm to grand design, and $9 \%$ changed from grand design to multi-arm or multi-arm to flocculent. Only two galaxies (NGC 3381 and NGC 5383) changed from grand design to flocculent going to $3.6 \mu \mathrm{m}$; seven galaxies changed from flocculent to grand design (NGC 1068, 3310, 3982, 4413, $4750,7714,7727)$. In all cases, the IR images have deeper exposures and better resolution (and less dust extinction) than the $B$-band images, so more structure is revealed.

\subsubsection{Extreme Spirals}

Arm classifications are a useful way of distinguishing different classes of spiral patterns, but like any aspect of galaxy structure, there are extremes even among these classes. In a large survey of galaxy images, there is a high probability of finding unusually strong examples of some aspects of galaxy morphology. One is the extremely oval inner rings recognized by Buta (2014) in the EFIGI SDSS optical galaxy sample (Baillard et al. 2011). These are SB rings which have an intrinsic minor-to-major axis ratio of $\leqslant 0.5$ compared to the average of $0.81 \pm 0.06$ (Buta 1995). Here we bring attention to "extreme spirals."

An "extreme spiral" is a grand design spiral having apparently large amplitude spiral arms of high pitch angle, and often showing so little background disk light that it is difficult to determine the orientation parameters of the system. Given the depth of $\mathrm{S}^{4} \mathrm{G} 3.6 \mu \mathrm{m}$ images, such systems are worthy of further investigation to determine how they form and how their properties differ from more normal spirals; most may be tidal in origin. We do not have a quantitative formal definition of such galaxies. They are merely recognized as being unusually strong and open compared to more average grand-design spirals.

Figure 29 shows six prominent examples from the $S^{4} G$ database:

NGC 3187-This galaxy is a member of a small group including NGC 3185, 3190, and 3193. One arm remains perpendicular to the apparent bar to a very large radius. The arms could be driven by an interaction, or, more likely, are due to the bar but perturbed by an interaction.

NGC 4488-This Virgo Cluster galaxy has a strong and highly unusual boxy bar-like zone from which two smooth and open arms emerge. It is not clear whether the arms and the apparent bar are coplanar features. Graham et al. (2012) have also noted a "bow-tie" aspect of the boxy zone of NGC 4488, and suggest the apparent arms may be due to an interaction.

$N G C$ 4572-This galaxy is a strong candidate for an extremely warped disk with considerable extraplanar material. The galaxy has a small nearby companion, and also has a similar redshift to that of a bright nearby elliptical galaxy, NGC 4589. Alternatively, it could be simply a strong tidal spiral, due to its possible local interaction. The Table 6 classification, Scd sp-superw?, brings attention to the warped disk possibility.

$N G C 4731$ - This appears to be an extreme late-type barred spiral. The bar is thin and likely is also flat (see also Figure 19). The spiral arms, however, are very open and show a strong right angle turn at large radius. The behavior is similar to NGC 4572, although we are not suggesting that NGC 4731 is another case of extreme warping.

IC 167-An exceptional case with a short bar and very strong spiral structure. At the NED distance of $41.6 \mathrm{Mpc}$, the diameter of the galaxy is $\approx 38 \mathrm{kpc}$.

$U G C$ 9057-An extreme late-type spiral with very open spiral arms, but not an extreme case of size.

\subsection{Recognizing Edge-on Magellanic Barred Spirals and the Special Case of NGC 4631}

The classification of NGC 4631 (de Vaucouleurs \& de Vaucouleurs 1964) sparked an interesting historical disagreement between Gerard de Vaucouleurs and Geoffrey Burbidge. De Vaucouleurs was well-known for classifying some edge-on galaxies with what appeared to be overly detailed types given what could actually be discerned in an image. The appearance of NGC 4631 led de Vaucouleurs to classify it as type SB(s)d, but the "B" and the "(s)" were nothing more than inferences based on the asymmetric shape. Burbidge et al. (1964) argued that no more than a type of Sc could be justified. Now, however, as we show in Figure 30, there is very likely a bar in NGC 4631 that is vertically thin. The feature was not clearly distinguishable in blue light. In Table 6, the adopted mid-IR classification is $\mathrm{SB}(\mathrm{s}) \mathrm{d}$ sp pec, confirming de Vaucouleurs's original interpretation.

The morphological aspects de Vaucouleurs used to assess the type of NGC 4631 can be used for other galaxies. For example, NGC 55 (Figure 30, top frame) is an almost edge-on late-type spiral classified as type $\mathrm{SB}(\mathrm{s}) \mathrm{m}$ sp in $\mathrm{RC} 3$. The galaxy is almost exactly edge-on, yet there is little doubt as to its classification. The brightness enhancement on the west side is likely to be a bar, and the asymmetric extension from this area is likely to be the single main spiral arm that is characteristic of type SB(s)m (de Vaucouleurs \& Freeman 1972). (The mean mid-IR classification for the galaxy in Table 6 is $\operatorname{SAB}(s) m$ sp.) In Figure 30, PGC 43970 is another 
Table 9

Arm Classifications for $1114 \mathrm{~S}^{4} \mathrm{G}$ Galaxies ${ }^{\mathrm{a}}$

\begin{tabular}{|c|c|c|c|c|c|c|c|c|c|}
\hline Galaxy & $\mathrm{AC}$ & Galaxy & $\mathrm{AC}$ & Galaxy & $\mathrm{AC}$ & Galaxy & $\mathrm{AC}$ & Galaxy & $\mathrm{AC}$ \\
\hline 1 & 2 & 3 & 4 & 5 & 6 & 7 & 8 & 9 & 10 \\
\hline NGC 45 & $\mathrm{~F}$ & NGC 899 & $\mathrm{~F}$ & NGC 1365 & $\mathrm{G}$ & NGC 2684 & $\mathrm{~F}$ & NGC 3185 & $\bar{G}$ \\
\hline NGC 63 & M & NGC 907 & $\mathrm{~F}$ & NGC 1367 & G & NGC 2701 & $\mathrm{~F}$ & NGC 3198 & M \\
\hline NGC 115 & $\mathrm{~F}$ & NGC 908 & M & NGC 1385 & $\mathrm{~F}$ & NGC 2710 & $\mathrm{G}$ & NGC 3206 & $\mathrm{~F}$ \\
\hline NGC 131 & $\mathrm{~F}$ & NGC 918 & M & NGC 1398 & M & NGC 2712 & M & NGC 3213 & F \\
\hline NGC 134 & M & NGC 941 & $\mathrm{~F}$ & NGC 1406 & G & NGC 2715 & $\mathrm{~F}$ & NGC 3225 & $\mathrm{~F}$ \\
\hline NGC 150 & G & NGC 986 & G & NGC 1415 & G & NGC 2731 & $\mathrm{~F}$ & NGC 3227 & G \\
\hline NGC 157 & $\mathrm{G}$ & NGC 988 & $\mathrm{~F}$ & NGC 1421 & $\mathrm{G}$ & NGC 2735 & G & NGC 3246 & $\mathrm{~F}$ \\
\hline NGC 178 & $\mathrm{~F}$ & NGC 991 & $\mathrm{~F}$ & NGC 1425 & M & NGC 2742 & M & NGC 3254 & $\mathrm{M}$ \\
\hline NGC 210 & $\mathrm{G}$ & NGC 1022 & G & NGC 1433 & G & NGC 2743 & $\mathrm{~F}$ & NGC 3259 & $\mathrm{M}$ \\
\hline NGC 247 & $\mathrm{~F}$ & NGC 1035 & $\mathrm{~F}$ & NGC 1436 & $\mathrm{G}$ & NGC 2748 & M & NGC 3264 & $\mathrm{~F}$ \\
\hline NGC 253 & G & NGC 1036 & $\mathrm{~F}$ & NGC 1438 & G & NGC 2750 & M & NGC 3274 & $\mathrm{~F}$ \\
\hline NGC 255 & $\mathrm{~F}$ & NGC 1042 & M & NGC 1452 & $\mathrm{G}$ & NGC 2770 & M & NGC 3287 & $\mathrm{~F}$ \\
\hline NGC 275 & $\mathrm{~F}$ & NGC 1051 & $\mathrm{~F}$ & NGC 1473 & $\mathrm{~F}$ & NGC 2776 & M & NGC 3294 & M \\
\hline NGC 289 & M & NGC 1068 & $\mathrm{G}$ & NGC 1483 & $\mathrm{~F}$ & NGC 2780 & G & NGC 3299 & $\mathrm{~F}$ \\
\hline NGC 298 & $\mathrm{~F}$ & NGC 1073 & M & NGC 1493 & $\mathrm{~F}$ & NGC 2805 & M & NGC 3310 & $\mathrm{G}$ \\
\hline NGC 300 & M & NGC 1076 & $\mathrm{~F}$ & NGC 1494 & $\mathrm{~F}$ & NGC 2841 & M & NGC 3319 & $\mathrm{G}$ \\
\hline NGC 337 & $\mathrm{~F}$ & NGC 1084 & $\mathrm{~F}$ & NGC 1512 & G & NGC 2854 & G & NGC 3320 & $\mathrm{M}$ \\
\hline NGC 337 A & $\mathrm{F}$ & NGC 1087 & $\mathrm{~F}$ & NGC 1515 & G & NGC 2856 & G & NGC 3321 & $\mathrm{M}$ \\
\hline NGC 360 & M & NGC 1090 & M & NGC 1518 & $\mathrm{~F}$ & NGC 2882 & M & NGC 3338 & M \\
\hline NGC 406 & $\mathrm{~F}$ & NGC 1097 & G & NGC 1519 & $\mathrm{~F}$ & NGC 2903 & M & NGC 3344 & $\mathrm{M}$ \\
\hline NGC 428 & $\mathrm{~F}$ & NGC 1179 & $\mathrm{~F}$ & NGC 1532 & $\mathrm{G}$ & NGC 2906 & M & NGC 3346 & $\mathrm{~F}$ \\
\hline NGC 450 & $\mathrm{~F}$ & NGC 1187 & M & NGC 1546 & G & NGC 2919 & G & NGC 3351 & $\mathrm{G}$ \\
\hline NGC 470 & M & NGC 1232 & M & NGC 1559 & $\mathrm{~F}$ & NGC 2938 & $\mathrm{~F}$ & NGC 3359 & M \\
\hline NGC 485 & G & NGC 1249 & $\mathrm{~F}$ & NGC 1566 & G & NGC 2964 & G & NGC 3361 & M \\
\hline NGC 488 & M & NGC 1253 & M & NGC 1637 & M & NGC 2964 & M & NGC 3364 & $\mathrm{~F}$ \\
\hline NGC 493 & M & NGC 1255 & $\mathrm{~F}$ & NGC 1640 & M & NGC 2967 & M & NGC 3368 & G \\
\hline NGC 514 & M & NGC 1258 & $\mathrm{~F}$ & NGC 1672 & $\mathrm{G}$ & NGC 2978 & G & NGC 3370 & M \\
\hline NGC 578 & M & NGC 1292 & $\mathrm{~F}$ & NGC 1679 & $\mathrm{~F}$ & NGC 2985 & M & NGC 3381 & $\mathrm{~F}$ \\
\hline NGC 600 & M & NGC 1299 & $\mathrm{~F}$ & NGC 1688 & M & NGC 3020 & $\mathrm{~F}$ & NGC 3389 & $\mathrm{~F}$ \\
\hline NGC 613 & M & NGC 1300 & $\mathrm{G}$ & NGC 1703 & M & NGC 3021 & M & NGC 3403 & M \\
\hline NGC 615 & M & NGC 1306 & $\mathrm{~F}$ & NGC 1792 & M & NGC 3023 & $\mathrm{~F}$ & NGC 3423 & $\mathrm{~F}$ \\
\hline NGC 628 & M & NGC 1309 & $\mathrm{~F}$ & NGC 1808 & G & NGC 3031 & G & NGC 3430 & M \\
\hline NGC 658 & M & NGC 1310 & M & NGC 1879 & $\mathrm{~F}$ & NGC 3041 & M & NGC 3433 & G \\
\hline NGC 672 & $\mathrm{~F}$ & NGC 1313 & $\mathrm{~F}$ & NGC 2104 & $\mathrm{~F}$ & NGC 3049 & G & NGC 3437 & M \\
\hline NGC 685 & $\mathrm{~F}$ & NGC 1325 & M & NGC 2460 & M & NGC 3055 & M & NGC 3445 & $\mathrm{~F}$ \\
\hline NGC 691 & M & NGC $1325 \mathrm{~A}$ & $\mathrm{~F}$ & NGC 2500 & $\mathrm{~F}$ & NGC 3057 & $\mathrm{~F}$ & NGC 3455 & $\mathrm{~F}$ \\
\hline NGC 701 & M & NGC 1337 & $\mathrm{~F}$ & NGC 2537 & $\mathrm{~F}$ & NGC 3061 & $\mathrm{~F}$ & NGC 3485 & M \\
\hline NGC 718 & G & NGC 1338 & M & NGC 2541 & $\mathrm{~F}$ & NGC 3066 & G & NGC 3486 & M \\
\hline NGC 723 & $\mathrm{~F}$ & NGC 1341 & $\mathrm{~F}$ & NGC 2543 & $\mathrm{G}$ & NGC 3147 & M & NGC 3488 & $\mathrm{~F}$ \\
\hline NGC 755 & $\mathrm{~F}$ & NGC 1345 & $\mathrm{~F}$ & NGC 2552 & $\mathrm{~F}$ & NGC 3153 & $\mathrm{~F}$ & NGC 3495 & M \\
\hline NGC 770 & M & NGC 1347 & $\mathrm{~F}$ & NGC 2604 & M & NGC 3155 & G & NGC 3504 & G \\
\hline NGC 772 & M & NGC 1350 & $\mathrm{G}$ & NGC 2608 & M & NGC 3162 & $\mathrm{M}$ & NGC 3507 & $\mathrm{G}$ \\
\hline NGC 803 & M & NGC 1353 & M & NGC 2633 & G & NGC 3169 & $\mathrm{~F}$ & NGC 3511 & M \\
\hline NGC 864 & M & NGC 1357 & M & NGC 2648 & G & NGC 3177 & G & NGC 3512 & M \\
\hline NGC 895 & M & NGC 1359 & $\mathrm{~F}$ & NGC 2681 & G & NGC 3184 & M & NGC 3513 & G \\
\hline NGC 3521 & M & NGC 3810 & M & NGC 4102 & M & NGC 4321 & G & NGC 4559 & M \\
\hline NGC 3547 & M & NGC 3813 & M & NGC 4106 & $\mathrm{G}$ & NGC 4348 & $\mathrm{G}$ & NGC 4561 & $\mathrm{~F}$ \\
\hline NGC 3549 & G & NGC 3846 A & $\mathrm{F}$ & NGC 4108 & $\mathrm{~F}$ & NGC 4351 & $\mathrm{~F}$ & NGC 4567 & M \\
\hline NGC 3583 & G & NGC 3850 & $\mathrm{~F}$ & NGC $4108 \mathrm{~B}$ & $\mathrm{~F}$ & NGC 4353 & $\mathrm{~F}$ & NGC 4568 & M \\
\hline NGC 3589 & $\mathrm{~F}$ & NGC 3876 & $\mathrm{~F}$ & NGC 4116 & $\mathrm{~F}$ & NGC 4376 & $\mathrm{~F}$ & NGC 4569 & G \\
\hline NGC 3596 & M & NGC 3887 & M & NGC 4120 & $\mathrm{~F}$ & NGC 4378 & $\mathrm{G}$ & NGC 4571 & $\mathrm{~F}$ \\
\hline NGC 3614 & M & NGC 3888 & M & NGC 4123 & M & NGC 4380 & M & NGC 4572 & $\mathrm{G}$ \\
\hline NGC 3622 & $\mathrm{~F}$ & NGC 3893 & M & NGC 4127 & M & NGC 4384 & $\mathrm{~F}$ & NGC 4579 & G \\
\hline NGC 3623 & G & NGC 3896 & $\mathrm{~F}$ & NGC 4133 & M & NGC 4385 & $\mathrm{~F}$ & NGC 4580 & M \\
\hline NGC 3625 & G & NGC 3898 & M & NGC 4136 & $\mathrm{~F}$ & NGC 4390 & $\mathrm{~F}$ & NGC 4591 & M \\
\hline NGC 3626 & G & NGC 3901 & $\mathrm{~F}$ & NGC 4141 & $\mathrm{~F}$ & NGC 4395 & $\mathrm{~F}$ & NGC 4592 & $\mathrm{~F}$ \\
\hline NGC 3627 & $\mathrm{G}$ & NGC 3906 & $\mathrm{~F}$ & NGC 4142 & $\mathrm{~F}$ & NGC 4396 & $\mathrm{~F}$ & NGC 4593 & $\mathrm{G}$ \\
\hline NGC 3629 & $\mathrm{~F}$ & NGC 3912 & $\mathrm{~F}$ & NGC 4145 & M & NGC 4409 & $\mathrm{~F}$ & NGC 4595 & $\mathrm{~F}$ \\
\hline NGC 3631 & M & NGC 3913 & $\mathrm{~F}$ & NGC 4152 & M & NGC 4411 A & M & NGC 4597 & $\mathrm{~F}$ \\
\hline NGC 3652 & G & NGC 3930 & $\mathrm{~F}$ & NGC 4157 & M & NGC $4411 \mathrm{~B}$ & M & NGC 4602 & M \\
\hline NGC 3654 & $\mathrm{~F}$ & NGC 3938 & M & NGC 4158 & M & NGC 4412 & G & NGC 4618 & $\mathrm{~F}$ \\
\hline NGC 3655 & M & NGC 3949 & $\mathrm{~F}$ & NGC 4159 & $\mathrm{~F}$ & NGC 4413 & G & NGC 4625 & $\mathrm{~F}$ \\
\hline NGC 3659 & $\mathrm{~F}$ & NGC 3953 & M & NGC 4162 & M & NGC 4414 & $\mathrm{~F}$ & NGC 4628 & $\mathrm{G}$ \\
\hline
\end{tabular}


Table 9

(Continued)

\begin{tabular}{|c|c|c|c|c|c|c|c|c|c|}
\hline Galaxy & $\mathrm{AC}$ & Galaxy & AC & Galaxy & $\mathrm{AC}$ & Galaxy & $\mathrm{AC}$ & Galaxy & $\mathrm{AC}$ \\
\hline 1 & 2 & 3 & 4 & 5 & 6 & 7 & 8 & 9 & 10 \\
\hline NGC 3664 & $\mathrm{~F}$ & NGC 3955 & $\mathrm{G}$ & NGC 4165 & $\mathrm{G}$ & NGC 4416 & $\mathrm{~F}$ & NGC 4630 & $\mathrm{~F}$ \\
\hline NGC 3672 & M & NGC 3956 & $\mathrm{~F}$ & NGC 4178 & $\mathrm{~F}$ & NGC 4428 & M & NGC 4632 & $\mathrm{~F}$ \\
\hline NGC 3673 & M & NGC 3972 & G & NGC 4180 & $\mathrm{G}$ & NGC 4430 & $\mathrm{~F}$ & NGC 4633 & $\mathrm{~F}$ \\
\hline NGC 3675 & M & NGC 3976 & M & NGC 4189 & $\mathrm{M}$ & NGC 4448 & M & NGC 4635 & $\mathrm{~F}$ \\
\hline NGC 3681 & M & NGC 3981 & $\mathrm{G}$ & NGC 4192 & $\mathrm{G}$ & NGC 4450 & $\mathrm{G}$ & NGC 4639 & M \\
\hline NGC $3683 \mathrm{~A}$ & M & NGC 3982 & $\mathrm{G}$ & NGC 4193 & M & NGC 4462 & G & NGC 4642 & M \\
\hline NGC 3684 & M & NGC 3985 & $\mathrm{~F}$ & NGC 4204 & $\mathrm{~F}$ & NGC 4470 & $\mathrm{~F}$ & NGC 4647 & $\mathrm{~F}$ \\
\hline NGC 3686 & M & NGC 3992 & M & NGC 4210 & $\mathrm{M}$ & NGC 4480 & M & NGC 4651 & M \\
\hline NGC 3687 & M & NGC 4020 & $\mathrm{~F}$ & NGC 4212 & $\mathrm{M}$ & NGC 4487 & $\mathrm{~F}$ & NGC 4653 & M \\
\hline NGC 3689 & $\mathrm{G}$ & NGC 4027 & $\mathrm{~F}$ & NGC 4216 & G & NGC 4490 & $\mathrm{~F}$ & NGC 4654 & M \\
\hline NGC 3691 & $\mathrm{~F}$ & NGC 4030 & M & NGC 4234 & $\mathrm{~F}$ & NGC 4496 A & $\mathrm{F}$ & NGC 4658 & $\mathrm{~F}$ \\
\hline NGC 3701 & M & NGC 4032 & $\mathrm{~F}$ & NGC 4237 & M & NGC 4498 & $\mathrm{~F}$ & NGC 4668 & $\mathrm{~F}$ \\
\hline NGC 3705 & M & NGC 4035 & $\mathrm{~F}$ & NGC 4238 & $\mathrm{~F}$ & NGC 4501 & M & NGC 4680 & G \\
\hline NGC 3715 & M & NGC 4037 & $\mathrm{~F}$ & NGC 4254 & M & NGC 4502 & $\mathrm{~F}$ & NGC 4682 & M \\
\hline NGC 3718 & G & NGC 4041 & M & NGC 4258 & $\mathrm{G}$ & NGC 4504 & M & NGC 4688 & $\mathrm{~F}$ \\
\hline NGC 3726 & M & NGC 4045 & M & NGC 4260 & $\mathrm{G}$ & NGC $4517 \mathrm{~A}$ & $\mathrm{~F}$ & NGC 4689 & M \\
\hline NGC 3730 & M & NGC 4049 & $\mathrm{~F}$ & NGC 4273 & M & NGC 4519 & $\mathrm{~F}$ & NGC 4701 & M \\
\hline NGC 3752 & $\mathrm{~F}$ & NGC 4050 & $\mathrm{G}$ & NGC 4276 & $\mathrm{~F}$ & NGC 4523 & $\mathrm{~F}$ & NGC 4713 & $\mathrm{~F}$ \\
\hline NGC 3755 & $\mathrm{~F}$ & NGC 4051 & M & NGC 4288 & $\mathrm{~F}$ & NGC 4525 & $\mathrm{~F}$ & NGC 4722 & M \\
\hline NGC 3756 & M & NGC 4062 & M & NGC 4294 & $\mathrm{~F}$ & NGC 4527 & $\mathrm{G}$ & NGC 4725 & $\mathrm{G}$ \\
\hline NGC 3769 & G & NGC 4067 & M & NGC 4298 & $\mathrm{~F}$ & NGC 4531 & G & NGC 4731 & $\mathrm{G}$ \\
\hline NGC 3780 & M & NGC 4080 & $\mathrm{~F}$ & NGC 4299 & $\mathrm{~F}$ & NGC 4534 & $\mathrm{~F}$ & NGC 4750 & $\mathrm{G}$ \\
\hline NGC 3782 & $\mathrm{~F}$ & NGC 4085 & M & NGC 4303 & M & NGC 4535 & M & NGC 4765 & $\mathrm{~F}$ \\
\hline NGC 3786 & G & NGC 4088 & M & NGC $4303 \mathrm{~A}$ & $\mathrm{~F}$ & NGC 4536 & M & NGC 4771 & M \\
\hline NGC 3788 & $\mathrm{G}$ & NGC 4094 & M & NGC 4313 & $\mathrm{G}$ & NGC 4540 & $\mathrm{~F}$ & NGC 4775 & $\mathrm{~F}$ \\
\hline NGC 3794 & $\mathrm{~F}$ & NGC 4096 & M & NGC 4314 & $\mathrm{G}$ & NGC 4545 & M & NGC 4779 & M \\
\hline NGC $3795 \mathrm{~A}$ & $\mathrm{~F}$ & NGC 4100 & $\mathrm{G}$ & NGC 4319 & G & NGC 4548 & $\mathrm{G}$ & NGC 4781 & $\mathrm{~F}$ \\
\hline NGC 4790 & $\mathrm{~F}$ & NGC 5236 & M & NGC 5597 & M & NGC 5954 & M & NGC 7163 & $\mathrm{G}$ \\
\hline NGC 4793 & M & NGC 5240 & M & NGC 5600 & $\mathrm{~F}$ & NGC 5956 & M & NGC 7167 & G \\
\hline NGC 4795 & G & NGC 5247 & $\mathrm{G}$ & NGC 5604 & M & NGC 5957 & $\mathrm{G}$ & NGC 7171 & G \\
\hline NGC 4800 & M & NGC 5248 & $\mathrm{G}$ & NGC 5624 & $\mathrm{~F}$ & NGC 5958 & $\mathrm{~F}$ & NGC 7184 & M \\
\hline NGC 4806 & M & NGC 5254 & M & NGC 5630 & $\mathrm{~F}$ & NGC 5962 & $\mathrm{~F}$ & NGC 7188 & G \\
\hline NGC 4808 & M & NGC 5289 & $\mathrm{G}$ & NGC 5633 & M & NGC 5963 & M & NGC 7191 & $\mathrm{G}$ \\
\hline NGC 4814 & M & NGC 5297 & M & NGC 5645 & $\mathrm{~F}$ & NGC 5964 & M & NGC 7205 & M \\
\hline NGC 4826 & M & NGC 5300 & $\mathrm{~F}$ & NGC 5660 & M & NGC 5970 & M & NGC 7218 & $\mathrm{~F}$ \\
\hline NGC 4897 & M & NGC 5313 & M & NGC 5661 & $\mathrm{G}$ & NGC 5985 & M & NGC 7247 & G \\
\hline NGC 4899 & M & NGC 5320 & M & NGC 5665 & $\mathrm{~F}$ & NGC 6015 & $\mathrm{~F}$ & NGC 7254 & G \\
\hline NGC 4900 & $\mathrm{~F}$ & NGC 5334 & $\mathrm{~F}$ & NGC 5667 & $\mathrm{~F}$ & NGC 6063 & M & NGC 7290 & M \\
\hline NGC 4902 & M & NGC 5336 & M & NGC 5668 & $\mathrm{~F}$ & NGC 6070 & M & NGC 7307 & $\mathrm{~F}$ \\
\hline NGC 4904 & $\mathrm{~F}$ & NGC 5339 & $\mathrm{G}$ & NGC 5669 & $\mathrm{~F}$ & NGC 6106 & $\mathrm{~F}$ & NGC 7314 & M \\
\hline NGC 4928 & $\mathrm{~F}$ & NGC 5346 & $\mathrm{~F}$ & NGC 5676 & M & NGC 6118 & M & NGC 7328 & $\mathrm{G}$ \\
\hline NGC 4942 & $\mathrm{~F}$ & NGC 5347 & G & NGC 5678 & $\mathrm{~F}$ & NGC 6140 & $\mathrm{~F}$ & NGC 7371 & M \\
\hline NGC 4948 A & $\mathrm{F}$ & NGC 5350 & $\mathrm{G}$ & NGC 5691 & $\mathrm{~F}$ & NGC 6155 & $\mathrm{~F}$ & NGC 7412 & M \\
\hline NGC 4951 & M & NGC 5362 & M & NGC 5693 & $\mathrm{~F}$ & NGC 6181 & $\mathrm{G}$ & NGC 7416 & $\mathrm{G}$ \\
\hline NGC 4961 & $\mathrm{~F}$ & NGC 5364 & M & NGC 5708 & $\mathrm{~F}$ & NGC 6207 & $\mathrm{~F}$ & NGC 7418 & M \\
\hline NGC 4965 & $\mathrm{~F}$ & NGC 5371 & $\mathrm{G}$ & NGC 5713 & $\mathrm{~F}$ & NGC 6217 & M & NGC $7418 \mathrm{~A}$ & $\mathrm{~F}$ \\
\hline NGC 4980 & $\mathrm{~F}$ & NGC 5375 & M & NGC 5740 & M & NGC 6236 & $\mathrm{~F}$ & NGC 7421 & M \\
\hline NGC 4981 & M & NGC 5376 & M & NGC 5744 & $\mathrm{G}$ & NGC 6237 & $\mathrm{~F}$ & NGC 7424 & M \\
\hline NGC 4995 & M & NGC 5383 & $\mathrm{~F}$ & NGC 5756 & $\mathrm{G}$ & NGC 6239 & $\mathrm{~F}$ & NGC 7437 & $\mathrm{~F}$ \\
\hline NGC 5002 & $\mathrm{~F}$ & NGC 5426 & M & NGC 5757 & M & NGC 6255 & $\mathrm{~F}$ & NGC 7448 & M \\
\hline NGC 5005 & M & NGC 5427 & G & NGC 5768 & M & NGC 6267 & $\mathrm{~F}$ & NGC 7456 & $\mathrm{~F}$ \\
\hline NGC 5012 & M & NGC 5430 & M & NGC 5774 & $\mathrm{~F}$ & NGC 6339 & M & NGC 7463 & $\mathrm{G}$ \\
\hline NGC 5016 & M & NGC 5443 & $\mathrm{G}$ & NGC 5783 & M & NGC 6395 & $\mathrm{~F}$ & NGC 7479 & G \\
\hline NGC 5033 & M & NGC 5448 & $\mathrm{G}$ & NGC 5789 & $\mathrm{~F}$ & NGC 6412 & M & NGC 7496 & $\mathrm{G}$ \\
\hline NGC 5042 & $\mathrm{~F}$ & NGC 5452 & $\mathrm{G}$ & NGC 5798 & $\mathrm{~F}$ & NGC 6434 & M & NGC 7497 & $\mathrm{~F}$ \\
\hline NGC 5054 & M & NGC 5457 & M & NGC 5806 & M & NGC 6870 & M & NGC 7513 & $\mathrm{G}$ \\
\hline NGC 5055 & M & NGC 5464 & $\mathrm{~F}$ & NGC 5832 & $\mathrm{~F}$ & NGC 6887 & M & NGC 7531 & M \\
\hline NGC 5068 & $\mathrm{~F}$ & NGC 5468 & M & NGC 5850 & G & NGC 6889 & $\mathrm{~F}$ & NGC 7537 & M \\
\hline NGC 5079 & $\mathrm{~F}$ & NGC 5474 & $\mathrm{~F}$ & NGC 5861 & M & NGC 6902 & M & NGC 7541 & M \\
\hline NGC 5085 & M & NGC 5476 & $\mathrm{~F}$ & NGC 5878 & M & NGC 6902 B & $\mathrm{F}$ & NGC 7552 & $\mathrm{G}$ \\
\hline NGC 5088 & $\mathrm{~F}$ & NGC 5480 & M & NGC 5879 & M & NGC 6923 & M & NGC 7582 & $\mathrm{G}$ \\
\hline NGC 5105 & $\mathrm{~F}$ & NGC 5486 & $\mathrm{~F}$ & NGC 5885 & M & NGC 6925 & M & NGC 7590 & M \\
\hline NGC 5109 & $\mathrm{~F}$ & NGC 5520 & M & NGC 5892 & M & NGC 7051 & M & NGC 7599 & M \\
\hline
\end{tabular}


Table 9

(Continued)

\begin{tabular}{|c|c|c|c|c|c|c|c|c|c|}
\hline Galaxy & $\mathrm{AC}$ & Galaxy & $\mathrm{AC}$ & Galaxy & $\mathrm{AC}$ & Galaxy & $\mathrm{AC}$ & Galaxy & $\mathrm{AC}$ \\
\hline 1 & 2 & 3 & 4 & 5 & 6 & 7 & 8 & 9 & 10 \\
\hline NGC 5112 & M & NGC 5534 & $\mathrm{G}$ & NGC 5894 & M & NGC 7059 & $\mathrm{~F}$ & NGC 7606 & M \\
\hline NGC 5116 & G & NGC 5560 & $\mathrm{G}$ & NGC 5899 & $\mathrm{G}$ & NGC 7070 & F & NGC 7625 & $\mathrm{~F}$ \\
\hline NGC 5117 & $\mathrm{~F}$ & NGC 5566 & G & NGC 5915 & $\mathrm{~F}$ & NGC 7091 & F & NGC 7661 & G \\
\hline NGC 5147 & $\mathrm{~F}$ & NGC 5569 & $\mathrm{~F}$ & NGC 5916 & G & NGC 7107 & F & NGC 7689 & M \\
\hline NGC 5169 & M & NGC 5577 & $\mathrm{~F}$ & NGC 5921 & M & NGC 7140 & M & NGC 7713 & $\mathrm{~F}$ \\
\hline NGC 5194 & $\mathrm{G}$ & NGC 5584 & M & NGC 5937 & M & NGC 7151 & $\mathrm{~F}$ & NGC 7714 & G \\
\hline NGC 5204 & $\mathrm{~F}$ & NGC 5585 & $\mathrm{~F}$ & NGC 5949 & $\mathrm{~F}$ & NGC 7154 & $\mathrm{~F}$ & NGC 7716 & M \\
\hline NGC 5205 & $\mathrm{G}$ & NGC 5587 & G & NGC 5950 & G & NGC 7162 & M & NGC 7721 & M \\
\hline NGC 5218 & M & NGC 5595 & M & NGC 5951 & $\mathrm{~F}$ & NGC $7162 \mathrm{~A}$ & $\mathrm{~F}$ & NGC 7723 & M \\
\hline NGC 7724 & G & IC 2007 & $\mathrm{~F}$ & UGC 1195 & $\mathrm{~F}$ & UGC 5897 & M & UGC 7943 & $\mathrm{~F}$ \\
\hline NGC 7727 & G & IC 2051 & M & UGC 1547 & $\mathrm{~F}$ & UGC 5922 & M & UGC 7950 & $\mathrm{~F}$ \\
\hline NGC 7741 & $\mathrm{~F}$ & IC 2056 & M & UGC 1551 & F & UGC 5934 & $\mathrm{~F}$ & UGC 8041 & F \\
\hline NGC 7743 & G & IC 2361 & $\mathrm{G}$ & UGC 1670 & F & UGC 5976 & F & UGC 8042 & F \\
\hline NGC 7750 & G & IC 2604 & $\mathrm{~F}$ & UGC 1753 & $\mathrm{~F}$ & UGC 5989 & $\mathrm{~F}$ & UGC 8052 & G \\
\hline NGC 7755 & M & IC 2627 & G & UGC 1862 & F & UGC 6023 & M & UGC 8053 & F \\
\hline NGC 7757 & M & IC 2969 & $\mathrm{~F}$ & UGC 2081 & $\mathrm{~F}$ & UGC 6104 & M & UGC 8056 & $\mathrm{~F}$ \\
\hline NGC 7764 & $\mathrm{~F}$ & IC 2995 & M & UGC 2443 & G & UGC 6157 & $\mathrm{~F}$ & UGC 8067 & $\mathrm{M}$ \\
\hline NGC 7793 & $\mathrm{~F}$ & IC 3102 & G & UGC 3070 & $\mathrm{~F}$ & UGC 6162 & $\mathrm{~F}$ & UGC 8084 & $\mathrm{~F}$ \\
\hline NGC 7798 & G & IC 3115 & G & UGC 4151 & M & UGC 6169 & G & UGC 8085 & $\mathrm{M}$ \\
\hline NGC 7817 & G & IC 3259 & $\mathrm{~F}$ & UGC 4169 & $\mathrm{~F}$ & UGC 6194 & G & UGC 8153 & $\mathrm{~F}$ \\
\hline IC 163 & $\mathrm{~F}$ & IC 3391 & $\mathrm{~F}$ & UGC 4238 & $\mathrm{~F}$ & UGC 6249 & $\mathrm{~F}$ & UGC 8282 & F \\
\hline IC 167 & $\mathrm{G}$ & IC 3392 & $\mathrm{G}$ & UGC 4390 & $\mathrm{~F}$ & UGC 6296 & $\mathrm{G}$ & UGC 8385 & F \\
\hline IC 529 & M & IC 3476 & $\mathrm{~F}$ & UGC 4499 & $\mathrm{~F}$ & UGC 6309 & G & UGC 8449 & F \\
\hline IC 600 & $\mathrm{~F}$ & IC 3517 & $\mathrm{~F}$ & UGC 4543 & $\mathrm{~F}$ & UGC 6320 & $\mathrm{~F}$ & UGC 8489 & F \\
\hline IC 718 & F & IC 3742 & $\mathrm{~F}$ & UGC 4549 & M & UGC 6335 & $\mathrm{G}$ & UGC 8516 & F \\
\hline IC 749 & F & IC 4216 & M & UGC 4621 & $\mathrm{G}$ & UGC 6345 & $\mathrm{~F}$ & UGC 8588 & $\mathrm{~F}$ \\
\hline IC 750 & G & IC 4221 & M & UGC 4623 & M & UGC 6517 & M & UGC 8597 & F \\
\hline IC 758 & $\mathrm{~F}$ & IC 4237 & M & UGC 4701 & $\mathrm{G}$ & UGC 6534 & $\mathrm{~F}$ & UGC 8658 & G \\
\hline IC 764 & $\mathrm{G}$ & IC 4351 & M & UGC 4714 & $\mathrm{~F}$ & UGC 6575 & $\mathrm{~F}$ & UGC 8733 & F \\
\hline IC 769 & G & IC 4407 & $\mathrm{~F}$ & UGC 4787 & $\mathrm{~F}$ & UGC 6670 & $\mathrm{~F}$ & UGC 8795 & G \\
\hline IC 776 & $\mathrm{~F}$ & IC 4468 & $\mathrm{G}$ & UGC 4824 & M & UGC 6713 & F & UGC 8851 & F \\
\hline IC 797 & F & IC 4536 & $\mathrm{~F}$ & UGC 4841 & M & UGC 6773 & F & UGC 8877 & F \\
\hline IC 800 & F & IC 4901 & M & UGC 4845 & G & UGC 6816 & F & UGC 8892 & F \\
\hline IC 851 & $\mathrm{~F}$ & IC 4986 & $\mathrm{~F}$ & UGC 4867 & $\mathrm{G}$ & UGC 6818 & F & UGC 8909 & F \\
\hline IC 863 & M & IC 5007 & $\mathrm{~F}$ & UGC 4871 & $\mathrm{~F}$ & UGC 6849 & F & UGC 8995 & $\mathrm{~F}$ \\
\hline IC 1014 & $\mathrm{~F}$ & IC 5039 & M & UGC 4922 & M & UGC 6879 & $\mathrm{~F}$ & UGC 9126 & $\mathrm{~F}$ \\
\hline IC 1055 & M & IC 5069 & M & UGC 4982 & M & UGC 6900 & $\mathrm{~F}$ & UGC 9215 & $\mathrm{~F}$ \\
\hline IC 1066 & $\mathrm{~F}$ & IC 5078 & M & UGC 5114 & $\mathrm{~F}$ & UGC 6903 & G & UGC 9274 & F \\
\hline IC 1067 & M & IC 5156 & M & UGC 5172 & $\mathrm{~F}$ & UGC 6917 & M & UGC 9291 & M \\
\hline IC 1125 & $\mathrm{~F}$ & IC 5201 & M & UGC 5228 & M & UGC 6930 & $\mathrm{~F}$ & UGC 9299 & $\mathrm{~F}$ \\
\hline IC 1151 & $\mathrm{G}$ & IC 5267 & M & UGC 5238 & $\mathrm{~F}$ & UGC 6983 & M & UGC 9310 & F \\
\hline IC 1158 & M & IC $5269 \mathrm{~A}$ & $\mathrm{~F}$ & UGC 5358 & G & UGC 7009 & $\mathrm{~F}$ & UGC 9569 & F \\
\hline IC 1251 & $\mathrm{~F}$ & IC $5269 \mathrm{~B}$ & M & UGC 5391 & $\mathrm{~F}$ & UGC 7133 & $\mathrm{~F}$ & UGC 9601 & F \\
\hline IC 1447 & M & IC $5269 \mathrm{C}$ & $\mathrm{F}$ & UGC 5478 & $\mathrm{~F}$ & UGC 7143 & M & UGC 9661 & F \\
\hline IC 1532 & $\mathrm{~F}$ & IC 5270 & $\mathrm{~F}$ & UGC 5522 & $\mathrm{~F}$ & UGC 7184 & M & UGC 9663 & F \\
\hline IC 1870 & F & IC 5271 & M & UGC 5612 & $\mathrm{~F}$ & UGC 7189 & $\mathrm{~F}$ & UGC 9730 & G \\
\hline IC 1892 & $\mathrm{~F}$ & IC 5273 & M & UGC 5646 & M & UGC 7239 & F & UGC 9746 & G \\
\hline IC 1914 & F & IC 5321 & $\mathrm{~F}$ & UGC 5676 & $\mathrm{~F}$ & UGC 7271 & F & UGC 9837 & M \\
\hline IC 1933 & F & IC 5325 & M & UGC 5688 & $\mathrm{~F}$ & UGC 7590 & $\mathrm{~F}$ & UGC 9858 & G \\
\hline IC 1952 & G & IC 5332 & M & UGC 5695 & $\mathrm{~F}$ & UGC 7690 & $\mathrm{~F}$ & UGC 9875 & F \\
\hline IC 1953 & $\mathrm{~F}$ & UGC 99 & $\mathrm{~F}$ & UGC 5707 & $\mathrm{~F}$ & UGC 7699 & $\mathrm{~F}$ & UGC 9936 & F \\
\hline IC 1954 & M & UGC 313 & $\mathrm{G}$ & UGC 5740 & $\mathrm{~F}$ & UGC 7700 & $\mathrm{~F}$ & UGC 9951 & F \\
\hline IC 1986 & $\mathrm{~F}$ & UGC 891 & $\mathrm{~F}$ & UGC 5832 & $\mathrm{~F}$ & UGC 7848 & M & UGC 10020 & F \\
\hline IC 1993 & M & UGC 1014 & $\mathrm{~F}$ & UGC 5841 & M & UGC 7911 & $\mathrm{~F}$ & UGC 10041 & F \\
\hline UGC 10054 & $\mathrm{~F}$ & ESO 287-37 & $\mathrm{F}$ & ESO 441-17 & $\mathrm{F}$ & ESO 550-24 & $\mathrm{F}$ & PGC 31979 & $\mathrm{~F}$ \\
\hline UGC 10290 & F & ESO 288-13 & $\mathrm{F}$ & ESO 442-13 & G & ESO 551-31 & $\mathrm{F}$ & PGC 32091 & M \\
\hline UGC 10310 & $\mathrm{~F}$ & ESO 289-26 & $\mathrm{G}$ & ESO 443-69 & M & ESO 572-18 & $\mathrm{F}$ & PGC 35705 & $\mathrm{~F}$ \\
\hline UGC 10413 & $\mathrm{G}$ & ESO 298-15 & $\mathrm{F}$ & ESO 443-80 & $\mathrm{F}$ & ESO 572-30 & $\mathrm{F}$ & PGC 36217 & F \\
\hline UGC 10437 & $\mathrm{~F}$ & ESO 298-23 & F & ESO 443-85 & M & ESO 576-1 & $\mathrm{G}$ & PGC 36274 & F \\
\hline UGC 10445 & $\mathrm{~F}$ & ESO 300-14 & $\mathrm{F}$ & ESO 445-89 & $\mathrm{F}$ & ESO 576-3 & M & PGC 36551 & $\mathrm{~F}$ \\
\hline UGC 10721 & M & ESO 305-9 & $\mathrm{F}$ & ESO 479-4 & $\mathrm{F}$ & ESO 576-17 & $\mathrm{F}$ & PGC 38250 & $\mathrm{~F}$ \\
\hline UGC 10736 & $\mathrm{~F}$ & ESO $340-42$ & $\mathrm{~F}$ & ESO 480-25 & $\mathrm{F}$ & ESO 576-32 & $\mathrm{F}$ & PGC 41965 & F \\
\hline UGC 10791 & $\mathrm{~F}$ & ESO $341-32$ & $\mathrm{~F}$ & ESO $481-18$ & M & ESO 576-50 & M & PGC 42868 & $\mathrm{~F}$ \\
\hline
\end{tabular}


Table 9

(Continued)

\begin{tabular}{|c|c|c|c|c|c|c|c|c|c|}
\hline Galaxy & $\mathrm{AC}$ & Galaxy & $\mathrm{AC}$ & Galaxy & $\mathrm{AC}$ & Galaxy & $\mathrm{AC}$ & Galaxy & $\mathrm{AC}$ \\
\hline 1 & 2 & 3 & 4 & 5 & 6 & 7 & 8 & 9 & 10 \\
\hline UGC 10803 & M & ESO $342-13$ & $\mathrm{~F}$ & ESO 482-35 & M & ESO 576-59 & $\mathrm{F}$ & PGC 43020 & $\mathrm{~F}$ \\
\hline UGC 10806 & $\mathrm{~F}$ & ESO $342-50$ & M & ESO 485-21 & G & ESO 580-22 & $\mathrm{F}$ & PGC 43345 & M \\
\hline UGC 12151 & $\mathrm{~F}$ & ESO $347-8$ & $\mathrm{~F}$ & ESO 502-20 & $\mathrm{F}$ & ESO 580-41 & $\mathrm{F}$ & PGC 44735 & $\mathrm{~F}$ \\
\hline UGC 12178 & $\mathrm{~F}$ & ESO 355-26 & M & ESO 503-22 & $\mathrm{F}$ & ESO 601-31 & $\mathrm{F}$ & PGC 44952 & $\mathrm{~F}$ \\
\hline UGC 12681 & F & ESO $357-12$ & $\mathrm{~F}$ & ESO 504-10 & F & ESO 602-30 & F & PGC 44954 & G \\
\hline UGC 12709 & F & ESO $358-20$ & $\mathrm{~F}$ & ESO 506-29 & F & PGC 6228 & M & PGC 45650 & M \\
\hline UGC 12732 & F & ESO $358-54$ & $\mathrm{~F}$ & ESO 507-65 & F & PGC 6244 & $\mathrm{~F}$ & PGC 45824 & $\mathrm{~F}$ \\
\hline UGC 12846 & $\mathrm{~F}$ & ESO $362-9$ & $\mathrm{~F}$ & ESO 508-7 & $\mathrm{F}$ & PGC 6626 & $\mathrm{~F}$ & PGC 45877 & M \\
\hline UGC 12856 & $\mathrm{~F}$ & ESO 400-25 & $\mathrm{F}$ & ESO 508-11 & $\mathrm{F}$ & PGC 6667 & $\mathrm{~F}$ & PGC 45958 & M \\
\hline ESO 12-10 & F & ESO 402-26 & G & ESO 508-19 & $\mathrm{F}$ & PGC 6667 & F & PGC 47721 & M \\
\hline ESO 13-16 & $\mathrm{F}$ & ESO 403-24 & F & ESO 508-24 & G & PGC 6898 & $\mathrm{~F}$ & PGC 48087 & M \\
\hline ESO 79-5 & F & ESO 407-9 & M & ESO 510-59 & $\mathrm{G}$ & PGC 11367 & M & PGC 52853 & $\mathrm{~F}$ \\
\hline ESO 79-7 & F & ESO 407-14 & M & ESO 532-22 & $\mathrm{F}$ & PGC 11744 & $\mathrm{~F}$ & PGC 53093 & M \\
\hline ESO $85-14$ & $\mathrm{~F}$ & ESO 408-12 & $\mathrm{F}$ & ESO 533-28 & M & PGC 12068 & F & PGC 53134 & $\mathrm{~F}$ \\
\hline ESO 85-30 & F & ESO 410-18 & $\mathrm{F}$ & ESO 539-7 & $\mathrm{F}$ & PGC 12608 & $\mathrm{~F}$ & PGC 53568 & $\mathrm{~F}$ \\
\hline ESO 116-12 & $\mathrm{F}$ & ESO 411-26 & $\mathrm{F}$ & ESO 541-4 & M & PGC 12633 & M & PGC 53779 & $\mathrm{~F}$ \\
\hline ESO 145-25 & F & ESO 418-8 & $\mathrm{F}$ & ESO 541-5 & $\mathrm{F}$ & PGC 12664 & $\mathrm{~F}$ & PGC 53796 & G \\
\hline ESO 150-5 & F & ESO 418-9 & F & ESO 544-30 & $\mathrm{F}$ & PGC 12981 & $\mathrm{~F}$ & PGC 54944 & $\mathrm{~F}$ \\
\hline ESO 187-35 & F & ESO 420-9 & F & ESO 545-2 & F & PGC 13684 & $\mathrm{~F}$ & PGC 66242 & $\mathrm{~F}$ \\
\hline ESO 187-51 & F & ESO 421-19 & F & ESO 545-16 & F & PGC 13716 & M & PGC 66559 & $\mathrm{~F}$ \\
\hline ESO 202-41 & $\mathrm{F}$ & ESO 422-5 & $\mathrm{F}$ & ESO 547-5 & $\mathrm{F}$ & PGC 14487 & $\mathrm{~F}$ & PGC 68061 & $\mathrm{~F}$ \\
\hline ESO 234-43 & F & ESO $422-41$ & F & ESO 548-5 & F & PGC 15869 & F & PGC 68771 & $\mathrm{~F}$ \\
\hline ESO 234-49 & F & ESO 423-2 & $\mathrm{F}$ & ESO 548-25 & G & PGC 16784 & F & PGC 69293 & $\mathrm{~F}$ \\
\hline ESO 237-52 & F & ESO 438-17 & M & ESO 548-32 & $\mathrm{F}$ & PGC 27616 & $\mathrm{~F}$ & PGC 69448 & M \\
\hline
\end{tabular}

\footnotetext{
${ }^{a}$ Arm classes: $\mathrm{F}$-flocculent, $\mathrm{M}-$ multi-armed, $\mathrm{G}$-grand design; all classifications are due to D. Elmegreen.
}

example of an edge-on galaxy that is likely to be a highly inclined $\mathrm{SB}(\mathrm{s}) \mathrm{m}$ Magellanic spiral. Many $\mathrm{SAB} / \mathrm{SBdm}$ and $\mathrm{SAB} / \mathrm{SBm} \mathrm{S}{ }^{4} \mathrm{G}$ galaxies have been recognized in this manner. When recognized in the edge-on view, there is an indication that $\mathrm{Sm}, \mathrm{Im}$ galaxies may be less flattened than, for example, $\mathrm{Sd}$ galaxies. The $S^{4} G$ provides an excellent database for reinvestigating the intrinsic flattenings of galaxies of different types.

\section{SUMMARY}

The $S^{4} G$ has provided a remarkable and valuable view of galaxy morphology in the mid-IR. Our main results from this study are as follows.

1. Using the precepts of CVRHS morphology, we give detailed classifications for more than 2400 nearby galaxies in the mid-IR. Together with paper I, this provides the first such examination of its kind in this wavelength domain. The classifications are based on two independent examinations of logarithmic, sky-subtracted images in units of mag $\operatorname{arcsec}^{-2}$. These are numerically combined to provide final mean classifications. Together with the paper I study, we also provide an atlas of images of all of the galaxies classified.

2. The dust-penetrated nature of mid-IR morphology allows us to see the full complement of stellar structures in galaxies, as per the original goal of the survey (Sheth et al. 2010). The main structures of CVRHS morphology are summarized in Table 1, and many are illustrated either in the montages or the atlas images accompanying this article. Other structures are described in the host of papers referenced in Section 1.

3. Figures 2-4 show that that CVRHS classifications of $S^{4} G$ galaxies have both good internal and external consistency. This consistency, however, does not preclude the existence of resolution and especially inclination biases in the catalog, independent of the sample bias against gas-poor galaxies. We have shown that the high-inclination half of the sample has higher frequencies of later-type and barred galaxies than does the lower-inclination half. For histograms and estimates of fractions of types, families, and varieties, we have adopted an inclination limit of $\approx 60^{\circ}$, based on the measured $3.6 \mu \mathrm{m}$ isophotal axis ratio at $\mu_{A B}$ $=25.5 \mathrm{mag} \operatorname{arcsec}^{-2}$. 


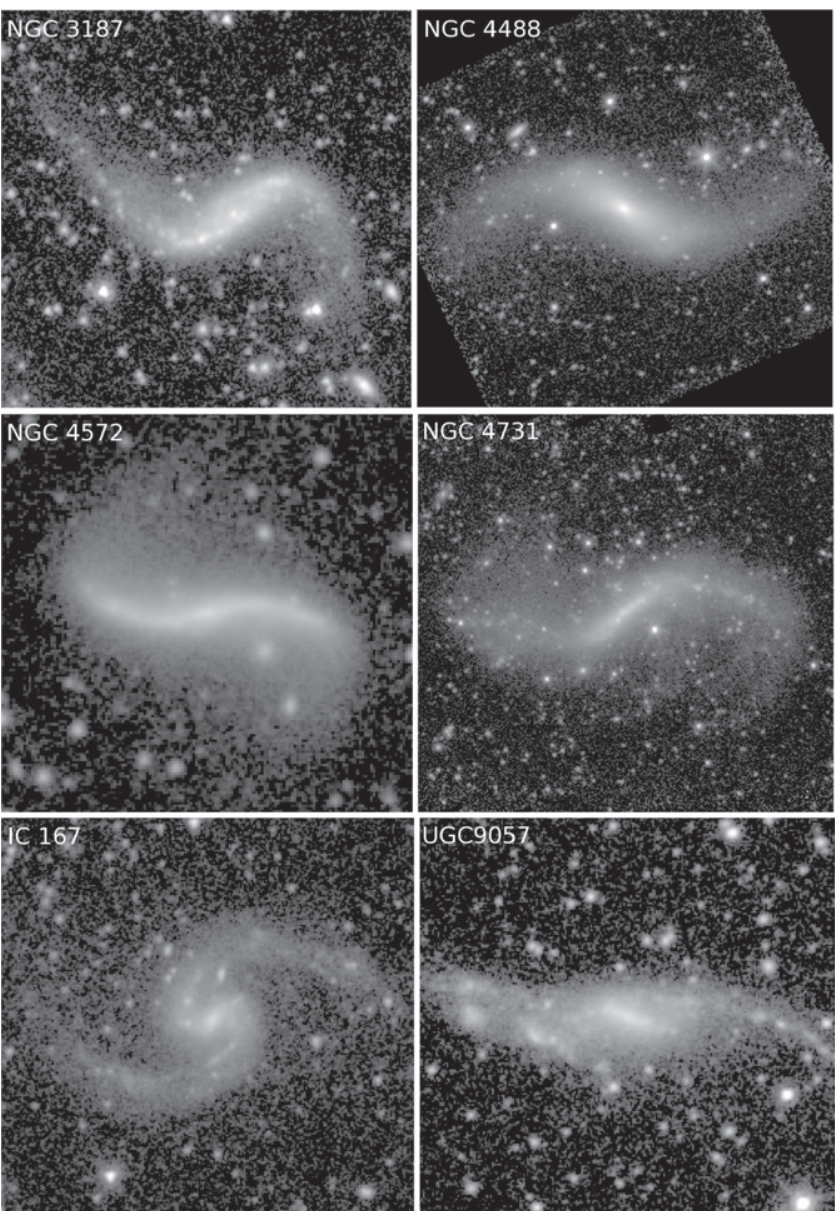

Figure 29. Six galaxies having what appears to be extremely open and extensive spiral structure. At least one (NGC 4572) could be a case of extreme warping (super-spw in Table 1).

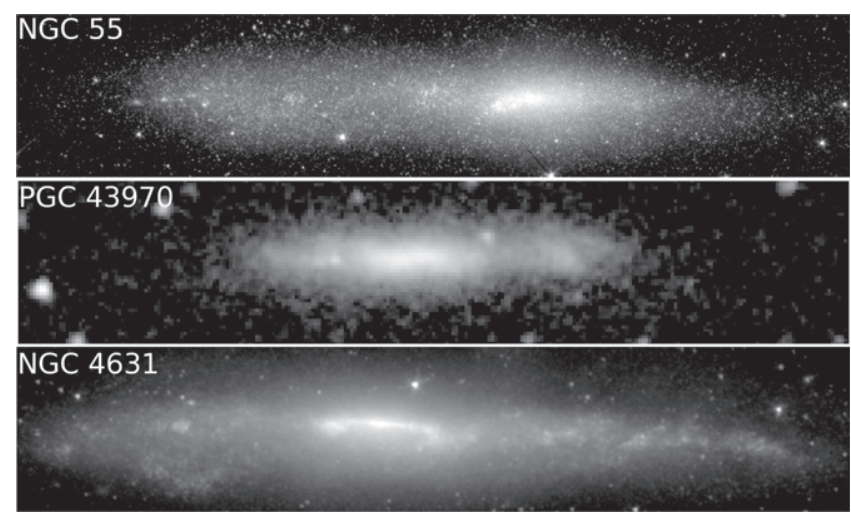

Figure 30. (Top two panels) two highly inclined late-type barred spirals showing the characteristic asymmetry of stage Sm (Magellanic). Bottom de Vaucouleurs used the appearance of NGC 4631 in blue light to deduce that it was a spiral of type $\mathrm{SB}(\mathrm{s}) \mathrm{d}$, even though the bar was not detectable in that band. At $3.6 \mu \mathrm{m}$, the bar is prominent.

4. Within this inclination limit, $48.5 \pm 1.4 \%$ of the galaxies in the $S^{4} G$ sample are what we call "extreme late-type galaxies," meaning galaxies in the type range Scd to Im. Scd-Sdm galaxies are essentially pure disk galaxies while some Sm and Im galaxies are also likely flat. The emphasis on these galaxies stems partly from the volume-limited
Table 10

Phase 3 CVRHS Classifications for $241 \mathrm{~S}^{4} \mathrm{G}$ Galaxies ${ }^{\mathrm{a}}$

\begin{tabular}{|c|c|c|c|}
\hline Galaxy & Type & Galaxy & Type \\
\hline 1 & 2 & 3 & 4 \\
\hline NGC 14 & $(\mathrm{~L}) \operatorname{IAB}(\mathrm{s}) \mathrm{m}$ & UGC 3070 & $\mathrm{~S} \underline{\mathrm{AB}}(\mathrm{s}) \mathrm{dm}$ \\
\hline UGC 156 & $\mathrm{SAB}(\mathrm{s}) \mathrm{m}$ & PGC 15869 & $\mathrm{SB}\left(\mathrm{R}^{\prime} 1, \mathrm{~s}\right) \mathrm{d}$ \\
\hline NGC 131 & $\mathrm{SAB}(\mathrm{r} \underline{\mathrm{r}}) \mathrm{dm}$ & NGC 1703 & $\mathrm{SA}(\mathrm{s}, \mathrm{nl}) \mathrm{c}$ \\
\hline NGC 157 & $\mathrm{SA}(\mathrm{s}) \mathrm{bc}$ & ESO 486-21 & IAm/Sph \\
\hline PGC 2689 & Im & ESO 422-41 & $\mathrm{S} \underline{\mathrm{A} B}(1) \mathrm{d}$ \\
\hline ESO 79-7 & $\mathrm{SB}(\mathrm{rs}) \mathrm{d}$ & IC 2135 & Scd sp/E8 \\
\hline ESO 541-5 & $\mathrm{SAB}(\mathrm{s}) \mathrm{m}$ & UGC 4148 & $\mathrm{SAB}(\mathrm{s}) \mathrm{m}$ \\
\hline UGC 711 & Scd $\mathrm{sp} / \mathrm{E}(\mathrm{d}) 9$ & UGC 4306 & $\mathrm{SA}(\mathrm{r}) 0^{+}$ \\
\hline UGC 891 & $\mathrm{SAB}(\mathrm{s}) \mathrm{m}$ & NGC 2604 & $\mathrm{SB}(\mathrm{rs}) \mathrm{cd}$ \\
\hline UGC 964 & $\mathrm{SAB}(\mathrm{s}) \mathrm{d} \mathrm{sp} / \mathrm{E}(\mathrm{d}) 6$ & UGC 4551 & $\mathrm{SA}(\mathrm{r}) 0^{+}$ \\
\hline UGC 1020 & $\mathrm{SAB}_{a} 0^{\circ}:$ & NGC 2683 & $(\mathrm{RL}) \mathrm{SA} \underline{B}_{a x}(\underline{\mathrm{r} s}) \mathrm{a}$ \\
\hline UGC 1133 & $\mathrm{dIm}$ & NGC 2712 & $\left(\mathrm{R}^{\prime}\right) \mathrm{SAB}_{a}(\mathrm{~s}, \mathrm{nl}) \mathrm{b}$ \\
\hline NGC 658 & $\mathrm{SA}(\mathrm{rs}) \mathrm{b}$ & NGC 2726 & $(\mathrm{RL}) \mathrm{SA}(\mathrm{rs}, \mathrm{rs}) \mathrm{a}$ \\
\hline NGC 672 & $\left(\mathrm{R}^{\prime}\right) \mathrm{SB}(\mathrm{s}) \mathrm{d}$ & NGC 2775 & $\mathrm{SA}(1, \underline{\mathrm{r}} \mathrm{s}) 0 / \mathrm{a}$ \\
\hline NGC 680 & $\mathrm{SA}(1) 0^{\circ}$ pec & UGC 4871 & $\mathrm{SAB}(\mathrm{s}) \mathrm{dm}$ \\
\hline NGC 723 & $\mathrm{SA}(\mathrm{s}) \mathrm{c}$ & NGC 2787 & $\mathrm{SAB}_{a}(\underline{\mathrm{r} s}, \mathrm{bl}) 0^{+}$ \\
\hline PGC 7682 & $\mathrm{Sdm} \mathrm{sp} / \mathrm{E}(\mathrm{d}) 6$ & UGC 4988 & $\mathrm{SAB}(\mathrm{s}) \mathrm{m}$ \\
\hline UGC 1670 & $\mathrm{~S} \underline{\mathrm{AB}}(\mathrm{s}) \mathrm{m}$ & NGC 2894 & $\mathrm{SA}(\mathrm{s}:) 0 / \mathrm{a}$ \\
\hline ESO 545-3 & Sc sp & UGC 5139 & $\mathrm{dIm}$ \\
\hline UGC 1862 & $\mathrm{SA}(\mathrm{s}) \mathrm{d}$ & UGC 5179 & $\mathrm{SA} 0^{\circ}$ \\
\hline UGC 1981 & $\mathrm{dIm}$ & NGC 2976 & $\mathrm{SA}(\mathrm{s}) \mathrm{d} / \mathrm{Sph}$ \\
\hline UGC 2082 & Scd $\mathrm{sp} / \mathrm{E}(\mathrm{d}) 8$ & NGC 3024 & $\mathrm{SB}(\mathrm{s}) \mathrm{m} \mathrm{sp}$ \\
\hline NGC 1051 & $\mathrm{SA} B(\mathrm{~s}) \mathrm{dm}$ & NGC 3027 & $\mathrm{SB}(\mathrm{s}) \mathrm{dm}$ \\
\hline NGC 1097 & $\begin{array}{l}\left(\mathrm{R}^{\prime}\right) \mathrm{SB}(\underline{\mathrm{r}} \mathrm{s}, \mathrm{nr}) \\
\quad \mathrm{ab} \text { pec }\end{array}$ & UGC 5354 & $\left(\mathrm{R}^{\prime}\right) \mathrm{SAB}(\mathrm{s}) \mathrm{d}$ \\
\hline ESO 356-18 & $\mathrm{SAB}(\mathrm{s}) \mathrm{m} \mathrm{sp}$ & UGC 5401 & $\mathrm{SAB}(\mathrm{s}) \mathrm{m}$ \\
\hline NGC 1187 & $\mathrm{SA} \underline{B}(\mathrm{rs}) \mathrm{bc}$ & NGC 3057 & $\mathrm{SB}(\mathrm{rs}) \mathrm{d}$ \\
\hline ESO 300-14 & $\mathrm{S} \underline{\mathrm{AB}}(\mathrm{rs}) \mathrm{cd}$ & UGC 5478 & $\mathrm{SAB}(\mathrm{s}) \mathrm{dm}$ \\
\hline NGC 1258 & $\begin{array}{l}\left(\mathrm{R}^{\prime} \mathrm{R}^{\prime}\right) \mathrm{SAB}(\mathrm{s}) \\
\quad \text { b pec }\end{array}$ & NGC 3177 & $\mathrm{SA}(\mathrm{rs}, \mathrm{rs}) \mathrm{b}$ \\
\hline PGC 12439 & Sc $\mathrm{sp} / \mathrm{E}(\mathrm{d}) 8$ & NGC 3182 & $\mathrm{SA}(\mathrm{r}) 0^{+}$ \\
\hline PGC 12608 & $\mathrm{SAB}(\mathrm{s}) \mathrm{dm}$ & UGC 5612 & $\left(\mathrm{R}^{\prime}\right) \mathrm{SB}(\mathrm{r} \underline{\mathrm{s}}) \mathrm{m}$ \\
\hline NGC $1316 \mathrm{C}$ & $\operatorname{SAB}_{a}(\mathrm{~s}) 0^{+}$ & NGC 3248 & $(\mathrm{RL}) \mathrm{SA}(1) 0^{\circ}$ \\
\hline NGC 1341 & (L)SB(rs)dm/Sph & UGC 5708 & $\mathrm{SB}(\mathrm{s}) \mathrm{d} \mathrm{sp}$ \\
\hline NGC 1347 & $\mathrm{SAB}_{a}(\mathrm{rs}) \mathrm{d}$ & NGC 3252 & Sc sp/E7 \\
\hline NGC 1357 & $\left(\mathrm{R}^{\prime} \mathrm{L}\right) \mathrm{SA}\left(\mathrm{r}^{\prime} 1, \underline{\mathrm{r}} \mathrm{s}\right) 0 / \mathrm{a}$ & NGC 3321 & $\mathrm{SA} \underline{B}(\mathrm{rs}) \mathrm{cd}$ \\
\hline IC 1962 & $\mathrm{SAB}(\mathrm{s}) \mathrm{m} \mathrm{sp}$ & UGC 5832 & $\left(\mathrm{R}^{\prime}\right) \mathrm{SB}(\mathrm{s}) \mathrm{m}$ \\
\hline NGC 1398 & $\left(\mathrm{R}^{\prime} \mathrm{R}\right) \mathrm{SB}(\underline{\mathrm{r}} \mathrm{s}, \mathrm{bl}) \mathrm{a}$ & UGC 5841 & $\mathrm{SB}(\mathrm{s}) \mathrm{ab}$ \\
\hline NGC 1433 & $\begin{array}{l}\left(\mathrm{R}_{1}{ }^{\prime}\right) \mathrm{SB}(\mathrm{p}, \mathrm{r}, \mathrm{bl}, \mathrm{nr} \\
\mathrm{nb}) \mathrm{a}\end{array}$ & UGC 5898 & $\mathrm{SAB}(\mathrm{s}) \mathrm{dm} \mathrm{sp}$ \\
\hline ESO 15-1 & $\mathrm{SB}(\mathrm{s}) \mathrm{m}$ & NGC 3395 & $\mathrm{SAB}(\mathrm{s}) \mathrm{c}$ pec \\
\hline PGC 90694 & $\mathrm{SBd} \mathrm{sp} / \mathrm{E} 8$ & NGC 3424 & $\operatorname{SAB}_{x}(1) \mathrm{a}$ \\
\hline IC 2051 & $\mathrm{SAB}(\underline{\mathrm{r} s}, \mathrm{nd}) \mathrm{b}$ & NGC 3447 & $\mathrm{SAB}(\mathrm{s}) \mathrm{dm}$ pec \\
\hline ESO 549-35 & $\mathrm{SAB}(\mathrm{s}) \mathrm{d}$ & NGC 3468 & $\mathrm{SA}(1) 0^{\circ}$ pec \\
\hline NGC $1511 \mathrm{~A}$ & $\mathrm{SAc} s p / \mathrm{E}(\mathrm{d}) 6-7$ & UGC 6112 & $\mathrm{~S} \underline{\mathrm{A} B}(\mathrm{~s}) \mathrm{d}$ \\
\hline NGC 1518 & $\mathrm{SB}(\mathrm{s}) \mathrm{dm}$ & ESO 502-16 & $\mathrm{SAB}(\mathrm{s}) \mathrm{m}$ \\
\hline PGC 14626 & $\mathrm{SB}(\mathrm{s}) \mathrm{dm}$ & ESO 502-20 & $\mathrm{S} \underline{\mathrm{AB}}(\mathrm{s}) \mathrm{cd}$ \\
\hline IC 2056 & $\mathrm{SA}(\mathrm{s}) \mathrm{b}$ & UGC 6271 & $\mathrm{SA}(1) 0^{+}[\mathrm{c}]$ \\
\hline UGC 6309 & $\left(\mathrm{R}_{12}^{\prime}\right) \mathrm{SB}(\mathrm{s}) \mathrm{bc}$ & NGC $4411 \mathrm{~B}$ & $\mathrm{SA}(\mathrm{s}) \mathrm{c}$ \\
\hline UGC 6345 & $\mathrm{SB}(\mathrm{s}) \mathrm{dm}$ & UGC 7557 & $\mathrm{SAB}(\mathrm{rs}) \mathrm{d}$ \\
\hline UGC 6390 & $\mathrm{Sd} \mathrm{sp}$ & UGC 7579 & $\mathrm{SB}(\mathrm{s}) \mathrm{m} \mathrm{sp} / \mathrm{Sph}$ \\
\hline NGC 3666 & $\mathrm{SA}(\mathrm{s}) \mathrm{bc}$ & UGC 7596 & $\mathrm{dIm}(\mathrm{dE}) / \mathrm{Sph}$ \\
\hline IC 2764 & $\mathrm{SA}(\mathrm{r}, \mathrm{nl}) 0^{\circ}$ & NGC 4461 & $\left(\mathrm{R}^{\prime} \mathrm{L}\right) \mathrm{SA}(\underline{\mathrm{r}} \mathrm{s}, 1) 0^{+}$ \\
\hline NGC 3701 & $\mathrm{SA}(\underline{\mathrm{r} s}) \mathrm{bc}$ & NGC 4489 & $\mathrm{SA}(\mathrm{rl}, \mathrm{nl}) 0^{\circ}$ \\
\hline UGC 6534 & $\mathrm{SA}(\mathrm{s}) \mathrm{cd}$ & NGC 4502 & $\mathrm{SAB}_{a}(\mathrm{~s}) \mathrm{cd}$ \\
\hline UGC 6570 & $\begin{array}{l}\mathrm{SA}(1) 0^{-}+ \\
\quad \mathrm{PR} \text { disk? }\end{array}$ & IC 3474 & Sdm sp \\
\hline NGC 3773 & $\mathrm{SA} 0^{-}$pec & NGC 4523 & $\mathrm{SAB}(\mathrm{s}) \mathrm{m}$ \\
\hline NGC 3795 & $\mathrm{SA}(\mathrm{s}) \mathrm{cd} \mathrm{sp}$ & NGC 4535 & $\mathrm{SAB}(\mathrm{s}) \mathrm{c}$ \\
\hline UGC 6682 & $\mathrm{SAB}(\mathrm{s}) \mathrm{m}$ & NGC 4548 & $\left(\mathrm{R}^{\prime}\right) \mathrm{SB}(\mathrm{rs}, \mathrm{bl}) \mathrm{a}$ \\
\hline NGC 3870 & $\mathrm{SB}_{a}\left(\mathrm{r}^{\prime} 1\right) 0^{\circ}[\mathrm{cd}]$ & UGC 7774 & $\mathrm{SB}(\mathrm{s}) \mathrm{dm} \mathrm{sp}$ \\
\hline
\end{tabular}


Table 10

(Continued)

\begin{tabular}{|c|c|c|c|}
\hline $\begin{array}{l}\text { Galaxy } \\
1\end{array}$ & $\begin{array}{l}\text { Type } \\
2\end{array}$ & $\begin{array}{c}\text { Galaxy } \\
3\end{array}$ & $\begin{array}{c}\text { Type } \\
4\end{array}$ \\
\hline NGC 3893 & $\mathrm{SA}(\mathrm{s}) \mathrm{c}$ & ESO 442-13 & $\mathrm{SAB}(\mathrm{s}) \mathrm{cd}$ \\
\hline IC 2963 & $\mathrm{Sdm}$ sp/E(d)7 & ESO 506-29 & $\mathrm{SB}(\mathrm{s}) \mathrm{dm}$ \\
\hline NGC 3930 & $\mathrm{SAB}(\mathrm{r} \underline{\mathrm{s}}) \mathrm{cd}$ & UGC 7848 & $\mathrm{SAB}_{a}(\mathrm{rs}) \mathrm{cd}$ \\
\hline ESO 440-27 & $\mathrm{Sc}$ sp/E(d)7-8 & NGC 4625 & $\mathrm{SAB}(\mathrm{r} \underline{\mathrm{r}}) \mathrm{m}$ \\
\hline PGC 37373 & $\mathrm{SAB}(\mathrm{s}) \mathrm{cd}$ & NGC 4636 & $\mathrm{E} 2$ \\
\hline NGC 3981 & $\mathrm{SAB}(\mathrm{s}) \mathrm{bc}$ pec & NGC 4654 & $\mathrm{SB}(\mathrm{rs}) \mathrm{cd}$ \\
\hline UGC 6956 & $\mathrm{SA} \underline{\mathrm{B}}(\mathrm{s}) \mathrm{m}$ & IC 3742 & $\mathrm{SB}(\mathrm{s}) \mathrm{m}$ \\
\hline UGC 6978 & $\mathrm{SB}(\mathrm{s}) \mathrm{m}$ & NGC 4684 & $\mathrm{SA}(1) 0^{-} \mathrm{sp}$ \\
\hline NGC 4037 & $\mathrm{SAB}(\mathrm{rs}) \mathrm{b}$ & NGC 4700 & $\mathrm{SB}(\mathrm{s}) \mathrm{m} \mathrm{sp}$ \\
\hline NGC 4051 & $\mathrm{SAB}(\mathrm{rs}, \mathrm{AGN}) \mathrm{bc}$ & UGC 7991 & Sd spw/E(d)8 \\
\hline NGC 4067 & $\mathrm{SB}(\mathrm{rs}) \mathrm{b}$ & NGC 4753 & ETG pec $/ \mathrm{S} 0^{\circ}$ pec \\
\hline IC 2996 & $\mathrm{SA}(\mathrm{rs}) \mathrm{bc}$ & NGC 4779 & $\mathrm{SA} \underline{\mathrm{B}}(\mathrm{rs}) \mathrm{b}$ \\
\hline NGC 4108 & $\left(\mathrm{R}^{\prime}\right) \mathrm{S} \underline{\mathrm{AB}}(\mathrm{rs}) \mathrm{bc}$ & NGC 4810 & $\mathrm{IBm}$ \\
\hline UGC 7125 & $\mathrm{SA} \underline{\mathrm{B}}(\mathrm{s}) \mathrm{m} \mathrm{sp}$ & NGC 4808 & $\mathrm{SAB}(\mathrm{rs}) \mathrm{cd}$ \\
\hline ESO 505-23 & $\mathrm{SB}(\mathrm{s}) \mathrm{d} \mathrm{sp}$ & PGC 44358 & $\mathrm{Sd} \mathrm{sp}$ \\
\hline UGC 7170 & $\mathrm{Sd} \mathrm{spw} / \mathrm{E}(\mathrm{d}) 8-9$ & PGC 44735 & $\mathrm{SAB}(\mathrm{rs}) \mathrm{dm}$ \\
\hline UGC 7184 & $\mathrm{SB}(\mathrm{rs}) \mathrm{dm}$ & PGC 44952 & $\mathrm{SA}(\mathrm{s}) \mathrm{cd}$ \\
\hline IC 769 & $\left(\mathrm{R}^{\prime}\right) \mathrm{SAB}(\mathrm{s}) \mathrm{bc}$ & NGC 4941 & $(\mathrm{RL}) \mathrm{SA}(\underline{\mathrm{r}} \mathrm{s}) \mathrm{a}$ \\
\hline UGC 7242 & $\mathrm{dIm}$ & IC 4182 & $\mathrm{SA}(\mathrm{s}) \mathrm{m}$ \\
\hline IC 3062 & $\mathrm{SA}(\mathrm{s}) \mathrm{bc}$ & NGC 4984 & $\left(\mathrm{R}^{\prime}, \mathrm{R}\right) \mathrm{SAB}_{a}(1, \mathrm{bl}, \mathrm{nl})$ \\
\hline NGC 4216 & $\begin{array}{l}\left(\mathrm{R}_{2}{ }^{\prime}\right) \mathrm{SAB}_{x a}(\mathrm{r}, \mathrm{nl}) \\
\quad \mathrm{ab} \mathrm{sp}\end{array}$ & ESO 576-3 & $\mathrm{SB}(\mathrm{rs}) \mathrm{dm}$ \\
\hline NGC 4235 & $\mathrm{SAB}_{\mathrm{x}}(0 / \mathrm{a} \mathrm{sp}$ & NGC 5016 & $\mathrm{~S} \underline{\mathrm{A}} \mathrm{B}(\underline{\mathrm{r}} \mathrm{s}) \mathrm{c}$ \\
\hline NGC 4248 & $\mathrm{dIm} \mathrm{sp} / \mathrm{Sph} \mathrm{pec}$ & PGC 45958 & $\mathrm{SA}(\mathrm{s}) \mathrm{c}$ \\
\hline NGC 4266 & $\mathrm{~S}^{\circ} \mathrm{sp} / \mathrm{E} 6$ & NGC 5054 & $\left(\mathrm{R}^{\prime}\right) \mathrm{SA}(\mathrm{s}, \mathrm{nr}) \mathrm{bc}$ \\
\hline NGC 4276 & $\mathrm{SA} \underline{\mathrm{B}}(\mathrm{r} \underline{\mathrm{r}}) \mathrm{d}$ & NGC 5073 & $\mathrm{SB}_{\mathrm{x}}(\mathrm{s}) 0 / \mathrm{a} \mathrm{sp}$ \\
\hline NGC 4286 & $\mathrm{dSA}(1) 0^{\circ}, N / \mathrm{Sph}$ & NGC 5088 & $\left(\mathrm{R}^{\prime}\right) \mathrm{SA}(\mathrm{s}) \mathrm{cd}$ \\
\hline NGC 4303 & $\mathrm{SAB}(\mathrm{rs}, \mathrm{nl}) \mathrm{bc}$ & NGC 5117 & $\mathrm{SA} \underline{\mathrm{B}}(\mathrm{s}) \mathrm{cd}$ \\
\hline NGC 4316 & $\mathrm{~S}(\mathrm{r}:) \mathrm{b}: \mathrm{sp} / \mathrm{E}(\mathrm{d}) 7$ & NGC 5147 & $\mathrm{SAB}(\mathrm{s}) \mathrm{c}$ \\
\hline NGC 4344 & $\mathrm{SA}(\mathrm{r}) 0^{+}[\mathrm{c}]$ & NGC 5170 & $\begin{array}{l}\left(\mathrm{R}^{\prime}\right) \mathrm{SAB}_{\mathrm{x}}(1) \text { a sp/E } \\
\text { (d) } 8-9\end{array}$ \\
\hline UGC 7490 & $\mathrm{SA}(\mathrm{s}) \mathrm{dm}$ & PGC 47721 & $\left(\mathrm{R}^{\prime}, \mathrm{R}\right) \mathrm{SA}(\mathrm{rs}) \mathrm{ab}$ \\
\hline IC 3298 & $\mathrm{SB}(\mathrm{s}) \mathrm{m} \mathrm{sp}$ & ESO 444-78 & $\mathrm{dIm}$ \\
\hline NGC 4389 & $\mathrm{SB}(\mathrm{rs}) \mathrm{ab}[\mathrm{d}]$ & UGC 8642 & $\mathrm{SB}(\mathrm{s}) \mathrm{m} \mathrm{sp}$ \\
\hline NGC 4396 & $\mathrm{SA}(\mathrm{s}:) \mathrm{c} \mathrm{sp}$ & UGC 8688 & $\operatorname{Im}$ \\
\hline ESO 577-38 & $\mathrm{dIm} \mathrm{sp}$ & NGC 6181 & $\mathrm{SA} B(\mathrm{rs}) \mathrm{bc}$ \\
\hline NGC 5337 & (L) $\mathrm{SB}(\mathrm{rs}) 0 / \mathrm{a}$ & NGC 6267 & $\mathrm{SB}(\mathrm{rs}) \mathrm{b}$ \\
\hline NGC 5339 & $\mathrm{SAB}(\mathrm{rs}, \mathrm{bl}) \mathrm{ab}$ & NGC 6339 & $\mathrm{SB}(\mathrm{s}) \mathrm{cd}$ \\
\hline NGC 5360 & Im sp/E6/Sph & IC 4901 & $\left(\mathrm{R}^{\prime}, \mathrm{R}^{\prime}\right) \mathrm{SB}(\underline{\mathrm{r}} \mathrm{s}) \mathrm{bc}$ \\
\hline ESO 510-26 & $\mathrm{SAB}(\mathrm{s}) \mathrm{m}$ & ESO $340-17$ & $\mathrm{SB}(\mathrm{s}) \mathrm{d}[0 / \mathrm{a}]$ pec \\
\hline NGC 5443 & $\left(\mathrm{R}^{\prime}\right) \mathrm{SA} \underline{B}_{\mathrm{x}}(\underline{\mathrm{r}} \mathrm{s}) \mathrm{a}$ & ESO 234-49 & $\mathrm{SA}(\mathrm{r}) \mathrm{cd}$ \\
\hline NGC 5474 & $\mathrm{SA}(\mathrm{s}) \mathrm{m}$ & ESO $341-32$ & $\mathrm{SA} \underline{\mathrm{B}}(\mathrm{rs}) \mathrm{dm}$ \\
\hline NGC 5476 & $\mathrm{~S} \underline{\mathrm{A}} \mathrm{B}(\mathrm{s}) \mathrm{c}$ & ESO 402-26 & $\left(\mathrm{R}_{1}^{\prime} \mathrm{R}_{2}^{\prime}\right) \mathrm{S} \underline{\mathrm{A}} \mathrm{B}(\underline{\mathrm{r}} \mathrm{s}, \mathrm{nl}) \mathrm{b}$ \\
\hline NGC 5526 & Sc sp/E7-8 & ESO 287-37 & $\mathrm{SAB}_{a}(\mathrm{~s}) \mathrm{dm}$ \\
\hline NGC 5560 & $\mathrm{SB}(\mathrm{s}) \mathrm{d}$ spw & ESO 288-13 & $\mathrm{SA} \underline{\mathrm{B}}(\mathrm{s}) \mathrm{dm}$ \\
\hline UGC 9206 & Im sp/Sph & NGC 7162 & $\mathrm{~S} \underline{\mathrm{A} B}(\underline{\mathrm{r}} \mathrm{s}) \mathrm{b}$ \\
\hline UGC 9245 & $\mathrm{SB}(\mathrm{r} \underline{\mathrm{r}}) \mathrm{dm}$ & ESO 601-7 & $\mathrm{SB}(\mathrm{s}) \mathrm{d} \mathrm{sp}$ \\
\hline UGC 9291 & $\mathrm{SAB}(\mathrm{rs}) \mathrm{c}$ & PGC 68061 & $\mathrm{Sb}$ sp pec/E7: \\
\hline NGC 5673 & $\mathrm{SB}(\mathrm{s}) \mathrm{d} \mathrm{sp}$ & ESO 532-32 & $\mathrm{dIm} \mathrm{sp}$ \\
\hline NGC 5668 & $\mathrm{~S} \underline{\mathrm{AB}} \mathrm{B}(\mathrm{rs}) \mathrm{cd}$ & ESO 602-3 & $\mathrm{SB}(\mathrm{s}) \mathrm{m} \mathrm{sp}$ \\
\hline NGC 5691 & $\mathrm{SB}(\mathrm{s}) \mathrm{dm}[0 / \mathrm{a}]$ & PGC 68771 & $\mathrm{SB}(\mathrm{s}) \mathrm{dm}$ \\
\hline NGC 5719 & $\left(\mathrm{R}^{\prime}\right) \mathrm{SA}(1) 0 / \mathrm{a} / \mathrm{E} 5-6$ & NGC 7314 & $\mathrm{SAB}(\mathrm{rs}) \mathrm{bc}$ \\
\hline ESO 580-22 & $\mathrm{SB}(\mathrm{s}) \mathrm{dm}$ & UGC 12151 & $\mathrm{SB}(\mathrm{rs}) \mathrm{d}$ \\
\hline PGC 52853 & $\mathrm{SA}(\mathrm{s}) \mathrm{cd}$ & ESO 346-1 & $\mathrm{Sd} \mathrm{sp} / \mathrm{E}(\mathrm{d}) 8$ \\
\hline IC 1066 & $\mathrm{SA}(\mathrm{s}) \mathrm{b}$ & IC 5267 & $\left(\mathrm{R}^{\prime}\right) \mathrm{SA}\left(\underline{\mathrm{r}} \mathrm{s}, \mathrm{r}^{\prime} \mathrm{l}\right) 0 / \mathrm{a}$ \\
\hline NGC 5798 & $\mathrm{SA}(\mathrm{s}) \mathrm{d}$ & IC $5269 \mathrm{C}$ & $\mathrm{SAB}(\mathrm{s}) \mathrm{dm}$ pec \\
\hline UGC 9661 & $\mathrm{SB}(\mathrm{s}) \mathrm{dm}$ & ESO 469-8 & $\mathrm{SAB}(\mathrm{s}) \mathrm{m} \mathrm{sp}$ \\
\hline PGC 91413 & $\mathrm{SB}(\mathrm{s}) \mathrm{dm} \mathrm{sp}$ & NGC 7537 & $\mathrm{SA}(\underline{\mathrm{r}} \mathrm{s}) \mathrm{b}$ \\
\hline NGC 5866 B & $\mathrm{dSA}(\mathrm{s}) 0 / \mathrm{a}[\mathrm{d}] / \mathrm{Sph}$ & NGC 7625 & $(\mathrm{~L}) \operatorname{SAB}(\mathrm{s}) 0 / \mathrm{a}[\mathrm{dm}]$ \\
\hline UGC 9815 & $\mathrm{SB}(\mathrm{s}) \mathrm{dm} \mathrm{sp}$ & ESO 240-4 & $\mathrm{SB}(\mathrm{s}) \mathrm{d} \mathrm{sp}$ \\
\hline
\end{tabular}

Table 10

(Continued)

\begin{tabular}{|c|c|c|c|}
\hline $\begin{array}{l}\text { Galaxy } \\
1\end{array}$ & $\begin{array}{c}\text { Type } \\
2\end{array}$ & $\begin{array}{c}\text { Galaxy } \\
3\end{array}$ & $\begin{array}{c}\text { Type } \\
4\end{array}$ \\
\hline NGC 5929 & E0 pec & IC 5334 & $\begin{array}{l}\left(\mathrm{R}^{\prime}\right) \mathrm{SA}(1) 0 / \mathrm{a} / \mathrm{E}(\mathrm{d}) \\
\quad 6-7\end{array}$ \\
\hline NGC 5963 & $\begin{array}{c}\mathrm{SAB}\left[\mathrm{rs}=\left(\mathrm{R}_{2}^{\prime}\right)\right. \\
\mathrm{SAB}(\mathrm{s}) \mathrm{c}] \mathrm{b}\end{array}$ & ESO 605-15 & $\mathrm{dIm} \mathrm{sp}$ \\
\hline NGC 5981 & $\mathrm{~S}^{+}{ }^{+} \mathrm{spw} / \mathrm{E}(\mathrm{d}) 7$ & NGC 7731 & $\begin{array}{l}\left(\mathrm{R}_{1} \mathrm{~L}, \mathrm{R}_{2}^{\prime}\right) \underline{S A B}_{a}(\mathrm{r}, \\
\mathrm{nl}) \mathrm{a}\end{array}$ \\
\hline UGC 9991 & $\mathrm{Sdm}$ & NGC 7755 & $\left(\mathrm{R}^{\prime}\right) \mathrm{SA} \underline{\mathrm{B}}(\mathrm{rs}, \mathrm{nr}) \mathrm{bc}$ \\
\hline NGC 6010 & $\mathrm{~S}^{-} \mathrm{sp} / \mathrm{E}(\mathrm{d}) 7$ & NGC 7793 & $\mathrm{SA}(\mathrm{s}) \mathrm{c}$ \\
\hline UGC 10290 & $\mathrm{SB}(\mathrm{s}) \mathrm{dm}$ & ............ & $\ldots$ \\
\hline
\end{tabular}

${ }^{\text {a }}$ Subsample is selected as every 10th galaxy from Table 6 .

nature of the survey, and partly from the fact that an $\mathrm{HI}$ radial velocity was used as a selection criterion.

5. Also within the adopted inclination limit, the barred CVRHS classification fraction, $f(F \geqslant 0.5)$, in the $\mathrm{S}^{4} \mathrm{G}$ depends on mid-IR type. For spiral galaxies in the mid-IR type range Scd-Sm, the fraction is $f(F \geqslant 0.5)=81.0 \pm$ $2.0 \%$, while over the type range $\mathrm{S} 0 / \mathrm{a}$ to $\mathrm{Sc}$, the fraction is $f(F \geqslant 0.5)=55.0 \pm 2.2 \%$ When examined further on a type by type basis, it appears that much of the drop for the S0/a to Sc subsample occurs for stages S $\underline{b c-S c}$. This drop does not appear to be present when $B$-band RC3 classifications are used for the same galaxies, and may be linked in part to the "earlier effect" in mid-IR classification where S0/a to Sc galaxies in blue light shift by $\approx$ one stage interval on average when classified in the $\mathrm{IR}$, while later $B$-band types do not.

The bars in mid-IR S0/a to Sc galaxies are typically not the same kinds of features as those in Scd-Sm galaxies. It is mainly the earlier-type galaxies that show bars having 3D box/peanut/X patterns, stellar ansae, or "barlenses." The bars in the later-type spirals are often linear chains of star-forming regions that are both azimuthally and vertically thin. This dichotomy in bar character has been known for a long time (e.g., Elmegreen \& Elmegreen 1985), and the distinction could be important for estimates of the cosmologically significant bar fraction (Sheth et al. 2008, 2014).

6. (s)-variety spirals average at a much later mid-IR stage (Scd) than do ring-lens variety spirals $\left(\mathrm{SO}^{+}\right.$to $\left.\mathrm{Sa}\right)$. Outer pseudorings are seen on average in $\mathrm{Sb}-\mathrm{Sbc}$ galaxies compared to $\mathrm{S}^{+}$to $\mathrm{S} 0 / \mathrm{a}$ for outer rings and lenses. The inner ring-lens fraction drops rapidly with advancing midIR stage.

7. Sph galaxies are well-represented in the $S^{4} \mathrm{G}$, although not all may have been recognized as such because these galaxies are best-defined using parameter correlations. Dwarf irregulars can show a regular elliptical background at $3.6 \mu \mathrm{m}$, and some early-type disk galaxies are actually bulgeless. These galaxies have been found by Kormendy \& Bender (2012) to have a natural "home" in a parallel sequence among extreme late-type galaxies. $\mathrm{S}^{4} \mathrm{G}$ morphology supports this interpretation. 

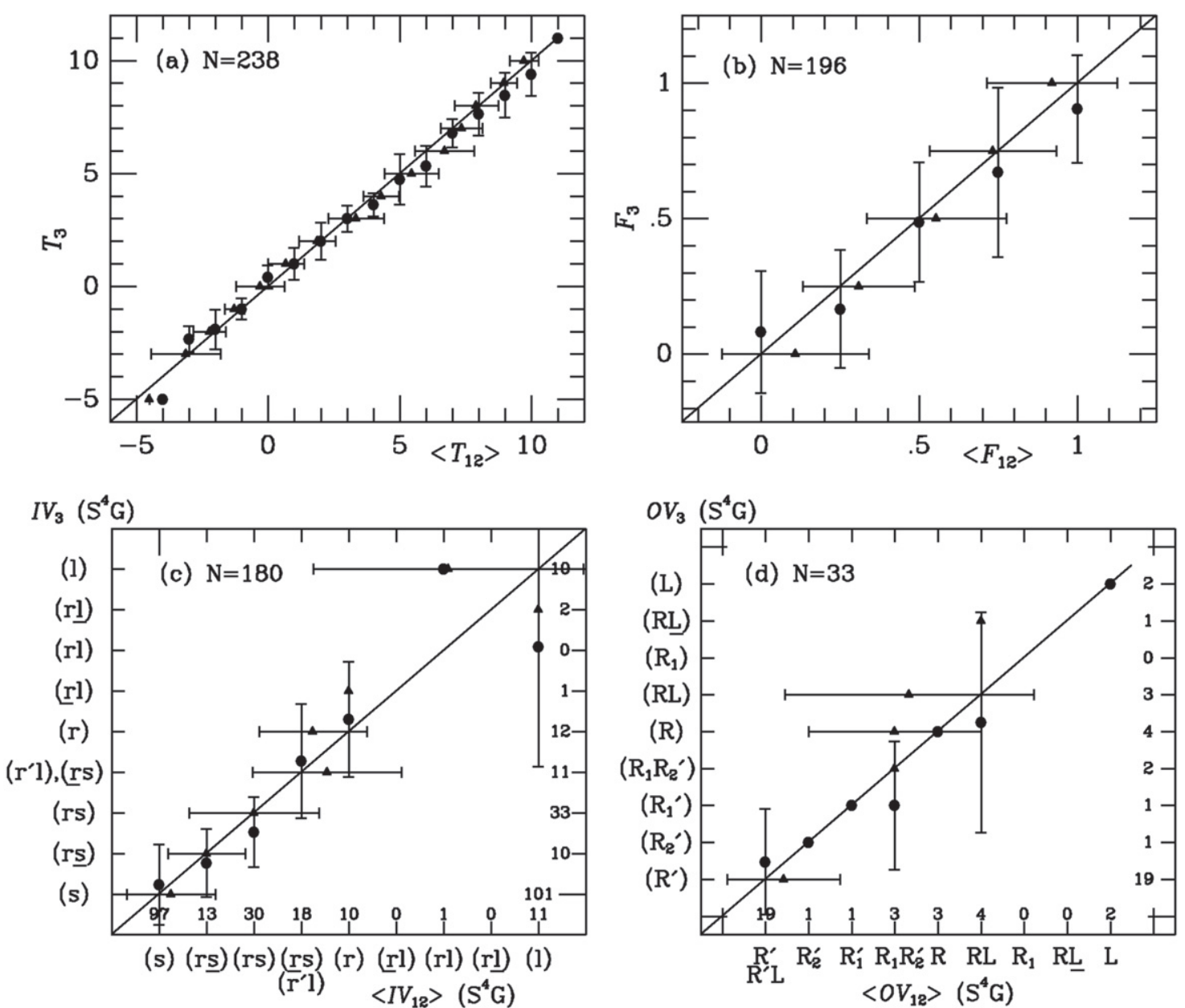

Figure 31. Comparisons between Phase 3 classifications (Table 10) and the mean classifications from Phases 1 and 2 given in Table 6. In (a) and (b), the comparisons use the numerical codes listed in Table 3. In (c) and (d), the comparisons use the letter classifications instead. Error bars are standard deviations in the system of the Table 3 codes in each case. The number $N$ in each panel is the number of features and includes multiple features in some cases. The number in each category is also given.

8. Grand design patterns are found in less than $20 \%$ of the classifiable spiral galaxies in the $\mathrm{S}^{4} \mathrm{G}$ catalog. The most common arm type is flocculent, owing to the sample emphasis on Scd-Sm stages.

9. The $S^{4} G$ sample includes many galaxies and morphological features worthy of follow-up studies. By "follow-up studies," we mean more detailed investigations of structure, such as using mass maps to approximate gravitational potentials, obtaining kinematic data, and correlating mid-IR structure with galaxy structure in optical and ultraviolet wavebands. Many follow-up studies have been made by the $S^{4} G$ team as listed in Section 1 . We have highlighted special cases of interest, and have in particular emphasized the numerous examples of apparently embedded disks in 3D early-type systems.

We thank the anonymous referee for many helpful comments that greatly improved this paper. R.B. acknowledges the support of a grant from the Research Grants Committee of the University of Alabama. K.S. acknowledges the support of the National Radio Astronomy Observatory. The National Radio Astronomy Observatory is a facility of the National Science Foundation operated under cooperative agreement by Associated Universities, Inc. J.H.K. acknowledges financial support from the Spanish MINECO under grant No. AYA2013-41243P. E.A. and A.B. acknowledge financial support to the DAGAL network from the People Programme (Marie Curie Actions) of the European Union's Seventh Framework Programme FP7/ 2007-2013/ under REA grant agreement number PITN-GA2011-289313. E.A. and A.B. also acknowledge financial support from the CNES (Centre National d'Etudes Spatiales -France). L.C.H. acknowledges support from the Kavli Foundation, Peking University, and the Chinese Academy of Science through grant No. XDB09030100 (Emergence of Cosmological Structures) from the Strategic Priority Research Program. This research has made use of the NASA/IPAC Extragalactic Database (NED), which is operated by the Jet Propulsion Laboratory, California Institute of Technology, under contract with the National Aeronautics and Space Administration.

\section{APPENDIX A PARTIAL PHASE 3 ANALYSIS}

The referee has suggested that we further assess the significance of the underline classifications in the mean stage, family, and variety classifications in Table 6 by carrying out a partial Phase 3 analysis for a random subset of $10 \%$ of the $S^{4} G$ 
sample. For this purpose, we reclassified every 10th galaxy in Table 6; the results are collected in Table 10. Figure 31 compares these classifications with those in Table 6. For all four comparisons, the results are very similar to those found between the Phase 1 and 2 classifications (Figures 2 and 3 ).

The partial Phase 3 analysis was used as follows. Table 5 gives $\sigma_{\langle 1,2\rangle}=\sigma\left(\Delta T_{\langle 1,2\rangle}\right)=\sqrt{\frac{\Sigma\left(T_{3}-\langle T\rangle\right)^{2}}{N-1}}$, where $T_{3}$ is the Phase 3 stage index and $\langle T\rangle$ is the mean of the Phase 1 and 2 stages in Table 6. The idea is to derive $\sigma\left(T_{3}\right)$ and compare it with $\sigma(T)$ from the Phase 1 and 2 analysis alone (Col. 2 of Table 5); we should find that $\sigma\left(T_{3}\right) \approx \sigma(T)$ if the classifications are consistent. For this purpose, we derive $\sigma\left(T_{3}\right)=\sqrt{\sigma\left(\Delta T_{\langle 1,2\rangle}\right)^{2}-\sigma(\langle T\rangle)^{2}}$. This gives $\sigma\left(T_{3}\right)=0.69$ of a stage interval, very similar to $\sigma(T)=0.74$ from the Phase 1 and 2 analysis alone. This supports our Phase 1,2 estimate of $\sigma(\langle T\rangle)=0.5$ stage interval and suggests that while the halfstep underline stage classifications are only marginally significant, it is still worth retaining them.

The partial Phase 3 analysis also supports our Phase 1, 2 assessment of the significance of the underline family classifications S $\underline{\mathrm{A}} \mathrm{B}$ and $\mathrm{SA} \underline{\mathrm{B}}$. Setting $\Delta F_{\langle 1,2\rangle 3}=F_{3}-\langle F\rangle$, we derive $\sigma\left(F_{3}\right)=0.79$ of a family interval compared to 0.68 of a family interval for Phases 1 and 2 alone (Table 5). This supports our Phase 1, 2 estimate of $\sigma(\langle F\rangle)=0.5$ family interval, indicating that the CVRHS sequence $\mathrm{SA}, \mathrm{S} \underline{\mathrm{AB}}, \mathrm{SAB}$, $\mathrm{SA} \underline{B}$, and $\mathrm{SB}$ is more internally significant than half-step stages.

For both inner and outer varieties, the internal standard deviations for the partial Phase 3 analysis are similar to those of the mean Phase 1, 2 classifications. Table 5 gives $\sigma\left(I V_{3}\right)$ $=1.19$ variety intervals compared to $\sigma(\mathrm{IV})=0.82$ of a variety interval from Phases 1 and 2 alone. This is reasonable agreement considering the size of the Phase 3 sample compared to the Phase 1, 2 sample. As for stages, the underline inner varieties also have marginal significance on the whole, but are still worth retaining in Table 6 because of the very good correlation for the standard inner varieties (s) to (r).

The same approach to outer varieties gives $\sigma\left(O V_{3}\right)=1.29$ outer variety intervals, which agrees well with $\sigma(\mathrm{OV})=1.27$ from the Phase 1,2 analysis in spite of the much smaller sample. Consistent classification of outer features appears to be more difficult than for stage, family, and inner variety, but nevertheless $\sigma(\langle\mathrm{OV}\rangle)=0.90$ (Table 5) supports the marginal significance of the multiple categories used.

\section{REFERENCES}

Athanassoula, E. 2005, MNRAS, 358, 1477

Athanassoula, E. 2012, in Secular Evolution of Galaxies XXIII Canary Islands Winter School of Astrophysicsed. J. Falcon-Barroso, \& J. H. Knapen (Cambridge: Cambridge Univ. Press), 305

Athanassoula, E., \& Bureau, M. 1999, ApJ, 522, 699

Athanassoula, E., Romero-Gómez, M., \& Masdemont, J. J. 2009, MNRAS, 394,67

Athanassoula, E., Romero-Gómez, M., Bosma, A., \& Masdemont, J. J. 2009, MNRAS, 400, 1706

Athanassoula, E., Laurikainen, E., Salo, H., \& Bosma, A. 2014, arXiv: 1405.6726

Aguerri, J. A. L., Méndez-Abreu, J., \& Corsini, E. M. 2009, A\&A, 495, 491

Baillard, A., Bertin, E., de Lapparent, V., et al. 2011, A\&A, 532, 75

Barazza, F., Jogee, S., \& Marinova, I. 2008, ApJ, 675, 1194

Binggeli, B., Sandage, A., \& Tammann, G. A. 1985, AJ, 90, 1681

Binney, J., \& Tremaine, S. 2008, Galactic Dynamics (2nd ed.; Princeton, NJ: Princeton Univ. Press)

Block, D. L., \& Puerari, I. 1999, A\&A, 342, 627
Briggs, F. H. 1990, ApJ, 352, 15

Burbidge, E. M., Burbidge, G. R., \& Prendergast, K. H. 1964, ApJ, 140, 1620 Bureau, M., \& Freeman, K. C. 1999, AJ, 118, 126

Bureau, M., Athanassoula, E., Chung, A., \& Aronica, G. 2004, in Penetrating Bars Through Masks of Cosmic Dusted. D. L. Block, et al. (Dordrecht: Springer), 139

Bureau, M., Aronica, G., Athanassoula, E., Dettmar, R.-J., Bosma, A., \& Freeman, K. C. 2006, MNRAS, 370, 753

Buta, R. 1984, PASA, 5, 472

Buta, R. 1995, ApJS, 96, 39

Buta, R. 2012, in Secular Evolution of Galaxies, XXIII Canary Islands Winter School of Astrophysicsed. J. Falcon-Barroso, \& J. H. Knapen (Cambridge: Cambridge Univ. Press), 155

Buta, R. 2013, in Planets Stars, and Stellar Systems ed. T. D. Oswalt, \& W. C. Keel, Vol. 6 (Dordrecht: Springer Science+Business Media), 1

Buta, R. 2014, in ASP Conf. Ser. 480, The Structure and Dynamics of Disk Galaxiesed. M. Seigar, \& P. Treuthardt (San Francisco, CA: ASP), 53

Buta, R., \& Combes, F. 1996, Galactic Rings Fund. of Cosmic Physics, 17, 95

Buta, R., \& Crocker, D. A. 1991, AJ, 102, 1715

Buta, R., \& Crocker, D. A. 1993, AJ, 106, 939

Buta, R. J., Corwin, H. G., \& Odewahn, S. C. 2007, The de Vaucouleurs Atlas of Galaxies (Cambridge: Cambridge Univ. Press)

Buta, R., Laurikainen, E., Salo, H., Block, D. L., \& Knapen, J. H. 2006, AJ, 132,1859

Buta, R., et al. 2010, ApJS, 190, 147

Buta, R., Laurikainen, E., Salo, H., \& Knapen, J. H. 2010, ApJ, 721, 259

Buta, R., Mitra, S., de Vaucouleurs, G., \& Corwin, H. G. 1994, AJ, 107, 118

Buta, R., Ryder, S. D., Madsen, G. J., et al. 2001, AJ, 121, 225

Cappellari, M., Emsellem, E., Krajnović, D., et al. 2011, MNRAS, 416, 1680

Casertano, S., Sackett, P. D., \& Briggs, F. 1991, Warped Disks and Inclined Rings Around Galaxies (Cambridge: Cambridge Univ. Press)

Campbell, L. A., et al. 2014, arXiv:1406.4867

Cisternas, M., et al. 2013, ApJ, 776, 50

Coe, D., Benítez, N., Sánchez, S. F., et al. 2006, AJ, 132, 926

Comerón, S. 2013, A\&A, 551, L4

Comerón, S., Knapen, J. H., Beckman, J. E., et al. 2010, MNRAS, 402, 2462

Comerón, S., Elmegreen, B. G., Knapen, J., et al. 2011a, ApJ, 741, 28

Comerón, S., Elmegreen, B. G., Knapen, J., et al. 2011b, ApJL, 738, L17

Comerón, S., Knapen, J., Sheth, K., et al. 2011c, ApJ, 729, 18

Comerón, S., Elmegreen, B. G., Salo, H., et al. 2012, ApJ, 759, 98

Comerón, S., Salo, H., Laurikainen, E., et al. 2014, A\&A, 562, 121

Contopoulos, G., \& Grosbol, P. 1989, A\&ARv, 1, 261

Crocker, D. A., Baugus, P. D., \& Buta, R. J. 1996, AoJS, 105, 353

Danby, J. M. A. 1965 , AJ, 70, 501

de Looze, I., et al. 2012, MNRAS, 427, 2797

de Vaucouleurs, G. 1959, HDP, 53, 275

de Vaucouleurs, G. 1963, ApJS, 8, 31

de Vaucouleurs, G., \& de Vaucouleurs, A. 1964, Reference catalog of Bright Galaxies Austin University of Texas Monographs in Astronomy No 1 (Austin, TX: Univ. of Texas Press)

de Vaucouleurs, G., de Vaucouleurs, A., \& Corwin, H. G. 1976, Second Reference Catalogue of Bright Galaxies Austim University of Texas Monographs in Astronomy No 2 (Austin, TX: Univ. of Texas Press)

de Vaucouleurs, G., de Vaucouleurs, A., Corwin, H. G., et al. 1991, in Third

Reference Catalogue of Bright Galaxies (New York: Springer)

de Vaucouleurs, G., \& Freeman, K. C. 1972, VA, 14, 163

de Zeeuw, P. T., et al. 2002, MNRAS, 329, 513

Elmegreen, B. G., \& Elmegreen, D. M. 1985, AoJ, 288, 438

Elmegreen, D. M. 1981, ApJS, 47, 229

Elmegreen, D. M., \& Elmegreen, B. G. 1982, MNRAS, 201, 1021

Elmegreen, D. M., \& Elmegreen, B. G. 1984, ApJS, 54, 127

Elmegreen, D. M., \& Elmegreen, B. G. 1987, ApJ, 314, 3

Elmegreen, D. M., Elmegreen, B. G., Marcus, M., Shahinyan, K., Yau, M., \& Petersen, M. 2009, ApJ, 701, 306

Elmegreen, D. M., Elmegreen, B. G., Yau, A., et al. 2011, ApJ, 737, 32

Elmegreen, D. M., Elmegreen, B. G., Erroz-Ferrer, S., et al. 2014, ApJ, 780, 32

Erwin, P. 2004, A\&A, 415, 941

Erwin, P., \& Debattista, V. P. 2013, MNRAS, 431, 3060

Eskridge, P. B., Frogel, J. A., Pogge, R. W., et al. 2000, AJ, 119, 536

Eskridge, P. B., Frogel, J. A., Pogge, R. W., et al. 2002, ApJS, 143, 73

Fazio, G. G., Hora, J. L., Allen, L. E., et al. 2004, ApJS, 154, 10

Graham, A. W. 2013, in Planets Stars, and Stellar Systemsed. T. D. Oswalt, \& W. C. Keel Vol. 6 (Dordrecht: Springer Science+Business Media), 91 Graham, A. W., Spitler, L. R., Forbes, D. A., et al. 2012, ApJ, 750, 121 Haynes, M. P., Jore, K. P., Barrett, E. A., Broeils, A., \& Murray, B. M. 2000, AJ, 120, 703 
Helou, G., Roussel, H., Appleton, P., et al. 2004, ApJS, 154, 253

Huertas-Company, M., Rouan, D., Tasa, L., Soucail, G., \& le Fèvre, O. 2008, A\&A, 478, 971

Holwerda, B., Munoz-Mateos, J.-C., Comeron, S., et al. 2014, ApJ, 781, 12

Hubble, E. 1926, ApJ, 64, 321

Hubble, E. 1936, The Realm of the Nebulae (Yale: Yale Univ. Press)

Ilyina, M., \& Sil'chenko, O. K. 2011, AstL, 37, 589

Ilyina, M., Sil'chenko, O. K., \& Afanasiev, V. L. 2014, MNRAS, 439, 334

Iodice, E., \& Corsini, E. M. 2013, in ASP Conf. Ser. 486, Multi-Spin Galaxies (San Francisco, CA: ASP)

Jedrzejewski, R. 1987, MNRAS, 226, 747

Jordán, A., et al. 2007, ApJS, 169, 213

Józsa, G. I. G., Oosterloo, T. A., Morganti, R., Klein, U., \& Erben., T. 2009, A\&A, 494, 489

Kendall, S., Kennicutt, R. C., Clarke, C., \& Thornley, M. 2008, MNRAS, 387, 1007

Kim, T., Sheth, K., Hinz, J. L., et al. 2012, ApJ, 753, 43

Kim, T., Gadotti, D. A., Sheth, K., et al. 2014, ApJ, 782, 64

Knapen, J. H. 2010, in Galaxies and their Masks, ed. D. L Block,

K. C. Freeman, \& I. Puerari (New York: Springer), 201

Knapen, J. H., Erroz-Ferrer, S., Roa, J., et al. 2014, A\&A, 569, 91

Kormendy, J. 1979, ApJ, 227, 714

Kormendy, J. 1984, ApJ, 286, 116

Kormendy, J. 2012, in Secular Evolution of Galaxies, XXIII Canary Islands Winter School of Astrophysics, ed. J. Falcon-Barroso, \& J. H. Knapen (Cambridge: Cambridge Univ. Press), 1

Kormendy, J., \& Bender, R. 1996, ApJL, 464, L119

Kormendy, J., \& Bender, R. 2012, ApJS, 198, 2

Kormendy, J., \& Kennicutt, R. C. 2004, ARA\&A, 42, 603

Kormendy, J., Fisher, D. B., Cornell, M. E., \& Bender, R. 2009, ApJS, 182, 216

Laine, S., Shlosman, I., Knapen, J. H., \& Peletier, R. F. 2002, ApJ, 567, 97

Laine, J., Laurikainen, E., Salo, H., et al. 2014a, MNRAS, 441, 1992

Laine, S., Knapen, J. H., Munoz-Mateos, J.-C., et al. 2014b, MNRAS, 444, 3015

Laurikainen, E., Salo, H., Buta, R., \& Knapen, J. 2009, ApJL, 392, L34

Laurikainen, E., Salo, H., Buta, R., Knapen, J., \& Comerón, S. 2010, MNRAS, 405, 1089

Laurikainen, E., Salo, H., Buta, R., \& Knapen, J. 2011, MNRAS, 418, 1452

Laurikainen, E., Salo, H., Athanassoula, E., et al. 2013, MNRAS, 430, 3489

Laurikainen, E., Salo, H., Athanassoula, E., Bosma, A., \& Herrera-Endoqui, M. 2014, MNRAS, L80

Lee, B., Giavalisco, M., Williams, C. C., et al. 2013, ApJ, 774, 47

Lin, C. C., \& Shu, F. H. 1966, PNAS, 55, 229

Luetticke, R., Dettmar, R.-J., \& Pohlen, M. 2000, A\&AS, 145, 405

Luetticke, R., Dettmar, R.-J., \& Pohlen, M. 2000, A\&A, 362, 435

Luetticke, R., Pohlen, M., \& Dettmar, R.-J. 2004, A\&A, 527, 539

Madore, B. F., Nelson, E., \& Petrillo, K. 2009, ApJS, 181, 572

Martin, P. 1996, in ASP conf. Ser. 91, Barred Galaxies, ed. R. Buta, D. A. Crocker, \& B. G. Elmegreen (San Francisco: ASP), 70
Matthews, L., \& de Grijs, R. 2004, AJ, 128, 137

Marinova, I., \& Jogee, S. 2007, ApJ, 659, 1176

Martinez-Valpuesta, I., Knapen, J. H., \& Buta, R. J. 2007, AJ, 134, 1863

Meidt, S., Schinnerer, E., Knapen, J. H., et al. 2012, ApJ, 744, 17

Meidt, S., Schinnerer, E., van de Venn, G., et al. 2014, ApJ, 788, 144

Munoz-Mateos, J., et al. 2013, ApJ, 771, 59

Munoz-Mateos, J., et al. 2014, submitted

Nair, P., \& Abraham, R. G. 2010, ApJL, 714, L260

Pahre, M., Ashby, M. L. N., Fazio, G. G., \& Willner, S. P. 2004, ApJS, 154,235

Paturel, G., Petit, C., Prugniel, P., et al. 2003, A\&A, 412, 45

Radburn-Smith, D. J., de Jong, R. S., Streich, D., et al. 2014, ApJ, 780, 105

Regan, M., \& Teuben, P. 2004, ApJ, 600, 595

Romero-Gómez, M., Masdemont, J. J., Athanassoula, E., \& García-Gómez, C. 2006, A\&A, 453, 39

Romero-Gómez, M., Athanassoual, E., Masdemont, J. J., \& García-Gómez, C. 2007, A\&A, 472, 63

Saha, K., de Jong, R., \& Holwerda, B. 2009, MNRAS, 396, 409

Salo, H., LauriKainen, E., Laine, J., et al. 2015, ApJS, in press

Sandage, A. 2005, ARAA, 43, 581

Sandage, A. 1961, The Hubble Atlas of Galaxies, Carnegie Inst. of Wash. Publ. No. 618

Sandage, A., \& Bedke, J. 1994, The Carnegie Atlas of Galaxies, Carnegie Inst. of Wash. Pub. No. 638

Sandage, A., \& Binggeli, B. 1984, AJ, 89, 919

Sandage, A., \& Tammann, G. A. 1981, A Revised Shapley-Ames Catalog, Carnegie Inst. of Wash. Pub. No. 635

Schwarz, M. P. 1981, ApJ, 247, 77

Schweizer, F., Whitmore, B. C., \& Rubin, V. C. 1983, AJ, 88, 909

Schweizer, F., Ford, W. K., Jedrzejewski, R., \& Giovanelli, R. 1987, ApJ, 320,454

Sellwood, J. 2013, in Planets Stars, and Stellar Systemsed. T. D. Oswalt, \&

G. Gilmore, Vol. 5 (Dordrecht: Springer Science+Business Media), 923

Sheth, K., Elmegreen, D. M., Elmegreen, B. G., et al. 2008, ApJ, 675, 1141

Sheth, K., Regan, M., Hinz, J. L., et al. 2010, PASP, 122, 1397

Sheth, K., Armus, L., Athanassoula, E. A., et al. 2013, Spitzer Proposal10043

Sheth, K. S ${ }^{4}$ G Team, 2014 AAS, 223, 205.02

Swardt, B., Sheth, K., Kim, T., et al. 2015, ApJ, submitted

Theys, J. C., \& Spiegel, J. C. 1976, ApJ, 208, 650

Thornley, M. 1996, ApJL, 469, L54

van den Bergh, S. 1976, ApJ, 206, 883

van den Bergh, S. 2009, ApJL, 694, L120

van Driel, W., Combes, F., Casoli, F., et al. 1995, AJ, 109, 942

Werner, M. W., Roellig, T. L., Low, F. J., et al. 2004, ApJS, 154, 1

Whitmore, B. C., Lucas, R. A., McElroy, D. B., et al. 1990, AJ, 100, 1489

Williams, R. E., Blacker, B., Dickinson, M., et al. 1996, AJ, 112, 1335

Zaritsky, D., Salo, H., Laurikainen, E., et al. 2013, ApJ, 772, 135

Zaritsky, D., Courtois, H., Munoz-Mateos, J.-C., et al. 2014, AJ, 147, 134 